University of Northern Colorado

Scholarship \& Creative Works @ Digital UNC

$5-2020$

\title{
An Examination of Current Leadership Practices in Earlychildhood and Early Childhood Special Education: A Mixed Methods Study
}

Sara Movahedazarhouligh

Follow this and additional works at: https://digscholarship.unco.edu/dissertations

\section{Recommended Citation}

Movahedazarhouligh, Sara, "An Examination of Current Leadership Practices in Earlychildhood and Early Childhood Special Education: A Mixed Methods Study" (2020). Dissertations. 656.

https://digscholarship.unco.edu/dissertations/656

This Text is brought to you for free and open access by the Student Research at Scholarship \& Creative Works @ Digital UNC. It has been accepted for inclusion in Dissertations by an authorized administrator of Scholarship \& Creative Works @ Digital UNC. For more information, please contact Jane.Monson@unco.edu. 
(C) 2020

SARA MOVAHEDAZARHOULIGH

ALL RIGHTS RESERVED 


\title{
UNIVERSITY OF NORTHERN COLORADO
}

Greeley, Colorado

The Graduate School

\section{AN EXAMINATION OF CURRENT LEADERSHIP PRACTICES IN EARLYCHILDHOOD AND EARLY CHILDHOOD SPECIAL EDUCATION: A MIXED METHODS STUDY}

A Dissertation Submitted in Partial Fulfillment of the requirements of the Degree of Doctor of Philosophy

Sara Movahedazarhouligh

\author{
College of Education and Behavioral Sciences \\ School of Special Education
}

May 2020 
This Dissertation by: Sara Movahedazarhouligh

Entitled: An Examination of Current Leadership Practices in Early Childhood and Early Childhood Special Education: A Mixed Methods Study

has been approved as meeting the requirements for the Degree of Doctor of Philosophy in the College of Education and Behavioral Sciences in the School of Special Education.

Accepted by the Doctoral Committee

John Luckner, Ed.D., Research Advisor

Rashida Banerjee, Ph.D., Committee Member

Jackie Davis, Ph.D., Committee Member

Dana Walker, Ph.D., Faculty Representative

Date of Dissertation Defense

Accepted by the Graduate School

Cindy Wesley, Ph.D.

Interim Associate Provost and Dean

Graduate School and International Admissions

Research and Sponsored Projects 


\begin{abstract}
Movahedazarhouligh, Sara. An Examination of Current Leadership Practices in Early Childhood and Early Childhood Special Education: A Mixed Methods Study. Published Doctor of Philosophy dissertation, University of Northern Colorado, 2020 .
\end{abstract}

Leadership is a vital component of any thriving system. Effective leadership is widely regarded as pivotal to the vitality of organizations. In early childhood (EC) and early childhood special education (ECSE) programs, strong leadership is particularly critical because directors and service providers are the gatekeepers of quality. Developing leadership among EC and ECSE professionals has become increasingly important as investing in $\mathrm{EC}$ education has been regarded as a public and private, national and international priority. However, the fields of EC and ECSE continue to struggle with the challenges of being overlooked when it comes to the leadership potentials, qualities, and challenges of leaders in the field. Yet, to date, little leadership literature and research is available for EC and ECSE professionals, in particular for those who are closer to practice.

This study aimed to investigate district-level and county-level EC and ECSE leadership practices in the state of Colorado to identify (a) who the leaders are in terms of their leadership qualifications and background, (b) the current leadership implementation state, (c) the challenges and barriers that affect the leaders' performance, and (d) the support these leaders need to practice quality leadership development and sustainability 
in the field. An explanatory sequential mixed-methods research approach was used to answer the enumerated questions. Two hundred ninety district-level and county-level EC and ECSE leaders from state or federally funded EC/ECSE programs participated in the online survey (Phase I), and 4 state-level EC/ECSE leaders participated in individual qualitative interviews (Phase II).

The findings of Phase I of this study provided valuable insight into districtlevel/county-level EC/ECSE leadership profiles, leadership implementation and barriers to leadership development in EC/ECSE as well as state and local and state leadership alignment and coordination. The hyper-feminine nature of the leadership at the local level, the lack of diversity among the leaders, and the absence of a specific EC/ECSE leadership license/endorsement which can be considered as the overarching theme from the other main findings in terms of leadership implementation, challenges, and professional development needs. In addition, the vague understanding and few opportunities for local directors to practice leadership vs. management, absence of any targeted leadership preparation in EC/ECSE, and lack of leadership development opportunities in Colorado that consequently impact local leaders' knowledge, competencies, skills, and expertise were other highlights among the findings.

These findings well-aligned with the Phase II state-level leaders' perceptions in terms of the absence of any formal preparation or specific certification/licensure in EC/ECSE leadership to prepare the local leaders for their leadership roles as the overarching barrier which results in a lack of strength in local quality leadership and leadership capacity building in the field. Other barriers that the state leaders mentioned included lack of accountability and rigor in leadership competencies, lack of 
collaboration within and across the systems, and multiple responsibilities for local leaders to deal with, which according to them, were all byproducts of the lack of preparation in leadership development specific to the fields of EC/ECSE.

This study adds to the scant literature in EC/ECSE leadership and provides support for future research and practice to further investigate leadership practices in EC/ECSE programs. The field of EC/ECSE is constantly changing as it continually seeks to improve the ways to serve children and families and support and strengthen the workforce and EC/ECSE systems. Growing public attention to the importance of high quality EC/ECSE programs has added to the pace of change (Kagan \& Kauerz, 2012). In these times of rapid change, it is critical to know how to lead effectively and to improve quality in ways that strengthen the field. There is no need to reinvent the wheel to fix the persistence challenges of quality leadership in the EC/ECSE sector. By applying lessons on leadership and change from across EC/ECSE and other disciplines, the field can learn how to support and nurture a strong, diverse, and resilient leadership and workforce to lead change, improvement, and innovations in EC/ECSE systems.

Keywords: leadership, leadership practices, early childhood, early childhood special education 


\section{ACKNOWLEDGEMENTS}

Above all, I thank you God. Thank you for your amazing power, for your great love and care, and for your countless blessings over my life. Thank you for the good and the bad throughout this journey far from home. Some were blessings and some were lessons. Thank you that you have brought hope through even the toughest of times, strengthening me for my purposes. I love you and I need you, this day and every day.

Thanks to my parents, Ali and Touba. Mom, Dad, thank you for the inspiration, drive and prayers! I am forever grateful for all the hardships you have been through to raise me to become the "Sara" that I am proud of today! This journey far from you was not easy, but here I am! I miss you every single day and counting seconds to be able to see you one day soon!

Thank you to my little man, my forever son, Parham. Mommy is so proud of raising you and so blessed for having you. I owe you your childhood years that you sacrificed so that I can complete this degree. I hope that this doctoral marathon will be a model for whatever journey you choose in your life. May we always choose to DREAM BIG and work harder than expected. I love you to the moon and back forever.

Thank you to my husband, Shahrooz, for your encouragement, patience and support. Without your love, understanding and sacrifice, I wouldn't be able to complete this degree. You deserve the best and I hope life will give us chances to keep building our days and tomorrows together. 
Thank you to two of the greatest people whose light seems to shine just a bit brighter than others; John Luckner and Rashida Banerjee. There would never be enough pages to adequately show my gratitude and appreciation to you. You are the true definition of wisdom, guidance and genuine support. Thank you for the support, sensitivity, encouragement, helping me in many occasions and making such a meaningful impact on my journey here. I am so grateful that you mentored me along the way and so honored to have worked with you and learned from you way beyond my doctoral degree program. You are and will always be in my prayers. God bless you and people like you who care and support.

And thank you to many others, my doctoral committee members, Dr. Jackie Davis and Dr. Dana Walker, my great friends that have always made me wonder what I have done to deserve them (Kaitlyn, Tara, Brittany, Dina, Lorae, and...), my professors in the School of Special Education (Silvia, Robin, Jackie, and..) and all who have been there for me with their love and understanding. 
TABLE OF CONTENTS

\section{CHAPTER}

I. INTRODUCTION

A Brief Conceptualization of Leadership in Early Childhood

Context

Theoretical Framework

Conceptual Framework

Statement of the Problem

Purpose of the Study

Significance of the Study

Research Questions

Definition of Terms

Summary of the Chapter

II. REVIEW OF LITERATURE.....

An Overview of Early Childhood and Early Childhood Special Education

Leadership in Early Childhood and Early Childhood Special

Education

Leadership Theories and Models

Whole Leadership Framework in Early Childhood Education

Other Leadership Models in Early Childhood

Application of Leadership Concepts to Early Childhood and

Early Childhood Special Education

Current Status of Early Childhood Leadership Preparation

Leadership Research Trends in Early Childhood and Early

Childhood Special Education

A Systematic Review of Leadership Research Trends in Early

Childhood and Early Childhood Special Education: A

Brief Report

Taking a Stance on National Leadership Trends, Issues, and Practices

Next Steps

Purpose of the Study

Summary of the Chapter 
III. METHODOLOGY ............................................ 60

Introduction

Early Childhood and Early Childhood Special Education in

Colorado

Purpose of the Study and Research Questions

Research Design

Participants and Sampling

Data Collection Procedures

Data Analysis Procedure

Summary of the Chapter

IV. FINDINGS

84

Purpose of the Study

Phase I: Quantitative Data Analysis-Descriptive Results

Results: Phase I

Phase I: Quantitative Data-Secondary Data Analysis Results

Phase II: Qualitative Data Analysis

Summary of the Chapter

V. DISCUSSION

Restatement of the Problem

Overview of the Study

Summary of the Findings and Implications

Limitations

Future Research

Conclusions

REFERENCES

APPENDIX

A. Phase I Online Survey.......................................... 186

B. Consent Form for the Online Survey ............................. 194

C. Interview Protocol................................................... 197

D. Consent Form for Phase II Individual Interviews.................... 200

E. Institutional Review Board Approval Letter........................... 203 


\section{LIST OF TABLES}

Table

1. DEC Recommended Practices in Leadership........................ 74

2. Participants' Demographic Characteristics by Gender, Age, Education, and Ethnicity................................... 87

3. Characteristics of the Participants' Current Leadership................. 89

4. Participants' Academic and Professional Characteristics............... 92

5. Highest Rated Leadership Practices in Terms of Leadership

Implementation............................................... 94

6. Highest Rated Leadership Practices in Terms of Implementation

Challenges................................................. 96

7. Highest Rated Leadership Practices in Terms of Professional

Development Needs in Implementation of Leadership Practices........ 98

8. Summary of the Responses to the Open-ended Questions............. 100

9. Reliability and Scale Statistics............................... 108

10. Extraction of Sums of Squared Loadings for Implementation of

Leadership Practices.......................................... 109

11. Pattern Matrix of the Extracted Loadings for Implementation of the Recommended Leadership Practices, Phase I Online Survey...........

12. Extraction of Sums of Squared Loadings for Challenges in

Implementation of the Recommended Leadership Practices. 
13 Pattern Matrix of the Extracted Loadings for Challenges in Implementation of the Recommended Leadership Practices, Phase I Online Survey ................................................

14. Extraction of Sums of Squared Loadings for the Levels of the

Professional Development Needs....................................

15. Pattern Matrix of the Extracted Loadings for Levels of Professional Development Needs in Implementation of Recommended Leadership Practices, Phase I Online Survey..........................................

16. Statistical Relationships Between Demographics and Recommended Leadership Practices......................................................

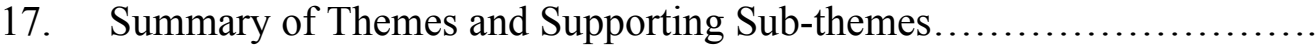




\section{LIST OF FIGURES}

1. Overview of Study Framework ................................... 69

2. Early Childhood/Early Childhood Special Education Leadership

Development Ecosystem........................................ 158 


\section{CHAPTER I}

\section{INTRODUCTION}

If your actions inspire others to dream more, learn more, do more and become more, you are a leader.

--John Quincy Adams

The importance of quality programs and services for young children is evident in the political agendas of many countries around the world (Davis, Krieg, \& Smith, 2015). This focus has been accompanied by increasing recognition that effective leadership in early childhood (EC) and early childhood special education (ECSE) programs makes a positive difference to the outcomes of children, families and communities (Davis et al., 2015; Hujala et al., 2016). Early childhood and ECSE programs are the contexts for how, as a society, we protect children, support families, and enable them to grow (Coleman, Sharp, \& Handscomb, 2016). In such contexts, contribution of leadership to improving organizational performance and raising child and family achievements remains unequivocal (Muijs, Aubrey, Harris, \& Briggs, 2004). However, there is a welldocumented lack of support for enhancing leadership capacity in the field of EC and ECSE (Bloom, 2014; Kagan \& Hallmark, 2001; Kagan \& Neuman, 1997; Talan, Bloom, \& Kelton, 2014).

Effective leadership in EC and ECSE programs is crucial, and the need has never been greater than at this time of increased accountability (Aubrey, 2007). Recognition 
and evidence of the value of EC and ECSE education has shifted the attention to the development of quality programs, and the need for effective leadership has become more critical (Division of Early Childhood [DEC], 2015; Talan, 2010; Talan et al., 2014). It is imperative to embrace the conviction that effective leadership is one necessary element of achieving high quality in EC and ECSE programs (Kivunja, 2015).

Leadership in EC and ECSE calls for commitment to creating a climate that promotes optimal growth and development of children as well as implementing the systems that ensure quality education is maintained (Talan et al., 2014). Such commitment lays the foundation that helps young children and their families develop into productive citizens in increasingly dynamically complex societies (Kivunja, 2015). This, according to Fullan (2001), is " the moral purpose of education" (p.4).

\section{A Brief Conceptualization of Leadership in Early Childhood Context}

Leadership in EC context is unlike leadership in other educational institutions. It requires unique administrative and managerial skills to plan, organize, lead, control, and direct the operations as well as leadership skills to provide an organizational vision, direction and acculturation (Kivunja, 2015). Allred and Hancock (2015) believed that a business or traditional model of leadership conflicts with many basic values of EC education. The business model emphasizes competition over collaboration and limits the potential for the EC workforce to see themselves as leaders in their daily work (Sullivan, 2010).

According to the literature and experts in the field, leaders of EC and ECSE programs have come, primarily, from within the ranks of the organization (Bloom \& Bella, 2005; Kagan \& Neuman, 1997; McCrea, 2015). In most cases, the leaders have 
served as educators in EC or ECSE programs before becoming the leader. However, unlike their kindergarten-12 (K-12) counterparts in school administration, EC and ECSE program leaders are, most often, viewed and described in terms of their administrative and managerial functions relating to the operation of the organization, rather than their leadership role which is concerned with the forward movement of the organization (Bloom, 2014; Kagan \& Bowman, 1997; Talan et al., 2014).

Rodd (2001) posited that EC leadership is made up of three elements: (a) technical knowledge and skills including pedagogical and curriculum leadership, (b) conceptual ability which involves critical thinking and advocacy, and (c) interpersonal skills. Bloom (2014) used a similar framework to that of Rodd (2001) when she categorized these skills into four areas: (a) communication skills, (b) decision-making and problem-solving skills, (c) interpersonal skills, and (d) organizational skills. Bloom (2014) also commented that defining leadership in terms of skills broadens the view of leadership to include those outside specific leadership positions.

The complexity inherent to the field of EC and ECSE where people and their relationships are at the center, requires a conception of leadership as an interpretive and situated phenomenon that is responsive to context (Murray \& Clark, 2013). According to Murray and Clark (2013), EC and ECSE settings are essentially dealing with human relationships in which there are layers and levels of responsibilities to the child, parents, staff, and the wider community. The interface of these relationships can generate uncertainty in how best to fulfill different responsibilities, where the needs or interests of the individuals compete with the interests of the whole group or where policy directives seem to be at odds with local needs. 
The Division for Early Childhood (DEC) of the Council for Exceptional Children's (CEC) position statement on leadership (DEC, 2015) emphasized by stating that leadership is not an individual's official role, title or sole authority within an organization. It is a process that, according to Yukl (2013), involves mutual influence and shared responsibility. The DEC is the largest organization that promotes policies and advances evidence-based practices that support families and enhances the optimal development of young children from birth through 8 who have or are at risk for developmental delays/disabilities in the United States.

To fulfill such perspective within the field, DEC (2014) has developed a set of recommended practices to provide guidance to practitioners about effective ways to promote the development of young children with or at risk for developmental delays/disabilities (DEC, 2014). These practices were developed to help translate research into practice and to ensure that children with or at risk for developmental delays/disabilities, their families, and the personnel who support them, have access to practices that result in better outcomes (Barton \& Smith, 2015). Leadership is one of the topic areas that provides guidance and includes 14 recommended practices that directly relate to the role of EC and ECSE leaders and administrators. According to Bruns, LaRocco, Sharp, and Sopko (2017), DEC-recommended practices offer a starting point for conceptualizing leadership in in the field.

\section{Theoretical Framework}

Ecological Systems Theory originated in Urie Bronfenbrenner's work during the 1940s on childhood and friendship patterns (Bronfenbrenner, 1995). According to Bronfenbrenner (1995), the ecological environment is conceived as a set of nested 
structures, each inside the next, that have continuing impacts on an individual's development. Within this structure are five layers, arranged from the closest to the individual to the farthest. These layers include: the microsystem, mesosystem, exosystem, macrosystem, and chronosystem (Bronfenbrenner, 1995). The microsystem represents an individual's immediate context including associated roles, actors, and environmental characteristics. The mesosystem consists of all other systems the individual frequents and his or her interrelationships. Individuals may also be influenced by contexts which are part of the exosystem or exists within a broader cultural system, the macrosystem, that dictates certain features of all associated systems. Lastly is chronosystem, which encompasses an individual's different reactions to environmental changes and determines how those changes will influence them (Bronfenbrenner, 1994).

The idea that individuals influence the people and institutions of their ecology as much as they are influenced by them has significant implications for the development of children (Toh, Jamaludin, Hung, \& Chua, 2014). Bronfenbrenner's (1994) Ecological Systems Theory has helped the field of EC understand child development in the context of the multiple environments and relationships around them. Children do not grow and learn in isolation, or only as the result of their parental or educational experiences. They are influenced by, and they actively influence the environment and relationships closest to them as well as those more distantly located. Bronfenbrenner's theory is best applied in developing a leader's understanding of individuals, actions, and interactions. In particular, understanding the mesosystem and exosystem can benefit leaders through understanding sources of service receivers' satisfaction or dissatisfaction. 
Bronfenbrenner's Ecological Systems Theory offers the idea that children's development takes place through the interrelationships between the various levels of environment they occupy and their interactions with others who form part of their environment. Unless those out-of-home environments are led by leaders with quality leadership competencies, efficacy and efficiency is less likely to be sustained. More specifically, the leaders act as a mediating layer to settle the interpretation of macro policies, benchmark them against the multilevel contexts they lead, make careful selection of innovations that they want to develop, translate them into micro implementation, consolidate the insights that arose from the processes, and re-strategize for innovation diffusion to other contexts (Toh et al., 2014).

Bronfenbrenner's (1994) Ecological Systems Theory also emphasized system thinking in a way that portrays how the ecosystems interact and influence one another, not how each acts independently or on its own. System thinking is hardly new. The field of early childhood education has long been familiar with it regarding children's development. When it comes to change and improvements, systems work in much the same way. Leaders do not work in isolations; rather they work in multiple contexts, all of which exert influence to varying degrees. When EC and ECSE programs try to change, they are influenced by the environments in which they operate. Their operation is shaped by the interactions among their context, community, and more distal political, economic, cultural, and social contexts.

\section{Ecological Leadership}

Contextual analysis of leadership in EC education and care draws attention to the operating environments of leadership which range from the circle closest to the leader-- 
the micro level--to the societal values and institutional structures that define leadership-the macro level. Between these extremes, we have the meso and exo levels. The meso level consists of the interaction or cooperation between the micro levels, while the exo level falls between the micro and macro levels and has an indirect effect on leadership (Wheatley, 2006).

Children and their families are an integral part of EC education practices. Without them, the whole organization could be closed down. Children utilize the educational and care-giving expertise available in the setting. Families utilize the setting as a source of educational or developmental support. The core of the ecological model of leadership is the contextual relationships within different levels of systems in EC education and the need for different roles and responsibilities of an EC leader to create and sustain interrelationships across different levels of the EC system. Leadership is always related, in one way or another, to the context and environment. In EC education, environment is defined in terms of the primary stakeholders that include children, families and professionals, mutual influences, and shared relationships. In such an environment, it is necessary to be conscious of effective and efficient leadership to promote a high-quality EC education that can result in positive outcomes in children's education, development, and well-being (Jennings \& Dooley, 2007).

In educational contexts, ecological leaders are able to create structures for fostering professional capital amongst teachers and enabling dialogue on the vision and implementations of the innovations at hand. Such leadership creates the structure for teacher experimentations, capacity building, and professionalism (e.g., through decentralized structures such as communities of practices, professional learning 
communities, and learning networks) and develops partnerships with industry, universities, and other schools in order to enable resources for teachers' experimentations and exploitations beyond the conventional constraints. In other words, an ecological leader examines the interplay between social-cultural meanings and physical-material affordances of innovations at the micro, meso, exo, macro, and chrono levels of a system (Jennings \& Dooley, 2007; Wheatley, 2006). More importantly, an ecological leader needs to go beyond system leadership and, according to Wheatley (2006), "act as brokers to form critical connections within and across the subsystems to bring social innovations and scale in the crucible of change for twenty-first century learning" (p.45).

\section{Conceptual Framework}

The conceptual framework that shapes the operational definition of "leadership" in this proposed study follows a leadership model developed by the National Association for Education of Young Children (NAEYC). The NAEYC proposed a model that expanded the roles and responsibilities of EC leaders. Drawing from Kagan and Bowman's seminal work (1997), Leadership in Early Care and Education, Kagan and Hallmark's work (2001), Cultivating Leadership in Early Care and Education: Reaping the Harvest of a New Approach to Leadership, and Lighting the Path: Developing Leadership in Early Education by Taba et al. (1999), the NAEYC proposed five suggested "functions" of leadership that are commonly found in EC settings and contexts. These five functions include: administrative leadership, community leadership, conceptual leadership, pedagogical leadership, and advocacy leadership (Kagan \& Bowman, 1997; Kagan \& Hallmark, 2001; Taba et al., 1999). 
According to Clifford (1997), EC leaders can be found at every level and can be characterized either through their positions (e.g., a president or director), skills (e.g., the act of influencing others), or characteristics (e.g., confidence or knowledge). It may be argued that it is because of such diverse aspects of leadership that the field of EC needs a broader definition that takes the many diverse leadership roles and behaviors into consideration. The NAEYC's proposed model of leadership in EC has been recommended by the Early Childhood Personnel Center (ECPC). The ECPC was established in January 2013. The Center is funded by the Department of Education's Office of Special Education Programs and serves as a national, multi-functional resource to address the challenges faced in the EC and ECSE workforce.

The DEC position statement on leadership (DEC, 2015) has also supported the five functions of leadership proposed by the NAEYC, stating that the five functions of leadership illustrate the necessity of building leadership capital at all levels of EC and ECSE service systems. According to the DEC (2015), this typology also suggests that leadership involves mutual influence and shared responsibility. Given the complexities of the EC and ECSE systems and the need for program administrators and practitioners to work collaboratively across program boundaries, the DEC believes the field should deliberately engage in identifying specific competencies necessary to be an effective leader as well as the organizational cultures necessary to promote and sustain highquality leadership (DEC, 2015).

The NAEYC's proposed leadership model has been conceptualized as Whole Leadership Framework in an EC context by the McCormick Center for Early Childhood Leadership at the National Louis University (Abel, Talan, \& Masterson, 2016). A 
detailed presentation of the Whole Leadership Framework will be provided in Chapter II of this study. An overview of the NAEYC's proposed model for leadership in EC context and the underpinnings of this model including: administrative leadership, community leadership, conceptual leadership, pedagogical leadership, and advocacy leadership is presented below.

\section{Administrative Leadership}

Culkin (1997) presented administrative leadership through two approaches: one of a manager, and one of a leader. She suggested that the responsibilities of a manager are to maintain a business or organization that provides a service such as budgeting, scheduling, and staff development and to go beyond the daily managerial tasks of running an EC program. Administrative leaders provide programs with vision, inspiration, structure, and direction (Culkin, 1997). According to Culkin (1997), the term leader implies a deeper and more far-reaching developmental relationship between persons within and outside the organization. Effective EC administrative leaders:

- Provide for ongoing learning for staff,

- Direct child and family services,

- Build internal and external communication networks, and

- Manage the program's finances (Culkin, 1997).

\section{Pedagogical Leadership}

Katz (1997) presented pedagogical leadership as a bridge between research and practice. Pedagogical leaders apply knowledge and information provided by researchers in their classrooms. Katz (1997) suggested that pedagogical leaders serve as interpreters of research and theory as well as disseminators of new information to parents and other 
teachers. Pedagogical leaders may shape the research agenda by making researchers aware of the current questions and issues within the classroom (Katz, 1997). Effective EC pedagogical leaders:

- Achieve and maintain credibility in both the practitioner and research worlds,

- Make their ideological assumptions about EC and the sources of these assumptions explicit,

- Help researchers and practitioners understand each other's perspectives and their contributions to the field of EC,

- Set the pedagogical agenda by maintaining an awareness of the issues in pedagogy, and

- Identify new developments in pedagogy (Katz, 1997).

\section{Community Leadership}

Crompton (1997) presented community leaders as those individuals who reach out to the community and communicate the importance of EC education for the wellbeing of children. While community leaders have a vision for change, they also have an understanding of the community and the individuals who live and work there. They go into the community to educate, inform, and build connections among families, available services, and resources (Crompton, 1997). Effective EC community leaders:

- Show the community that quality EC education significantly affects children's later success in school and in life,

- Convince other community leaders to place EC education prominently in the community agenda, 
- Influence community policies to create quality EC programs by outlining what needs to be done, and

- Assist in securing the human and financial resources needed for quality EC programs (Crompton, 1997).

\section{Advocacy Leadership}

Blank (1997) considered advocacy leaders to be those who have a vision to bring about change for children, families, and programs. In this perspective, advocacy leaders need to build on their vision with long-term planning and forward thinking. These leaders also have knowledge about the legislative process and commonly reach out and work with other disciplines (Blank, 1997). Effective advocacy EC leaders:

- Have a vision for what they believe children need to grow and thrive,

- Are able to communicate this vision and plan for the long-term,

- Use data, time, and resources strategically,

- Are persistent and know how and when to compromise, and

- Work collaboratively with colleagues (Blank, 1997).

\section{Conceptual Leadership}

Kagan and Neuman (1997) presented conceptual leadership as leadership that goes "beyond thinking about individual programs and having a sense of the field as a whole" (p. 59). They also suggested that conceptual leaders are open to diverse perspectives, think long-term, and want to bring about change. Kagan and Neuman (1997) also stated that conceptual leadership focuses on improving EC education to a more just and equitable society. Effective conceptual EC leaders: 
- Think about the field of EC as a whole, rather than as individual programs,

- Think together by collaborating with others in the field,

- Are responsive to diverse perspectives,

- Look towards the future and consider possibility, and

- Reach out to other institutions and their leaders, families, and communities and seek to impact the social good by considering how EC contributes to society (Kagan \& Neuman, 1997).

The aforementioned leadership underpinnings highlight the fact that the people, the places, and the practices that an EC or ECSE leader has to lead involve intricate structural and cultural dynamics, different from a school context which is more advanced than the EC and ECSE settings. It looks different, it involves different roles, and the leader has to perform functions that are significantly different from those in a primary or secondary school or an institution (Crompton, 1997; Culkin, 1997; Kagan \& Hallmark, 2001; Kagan \& Neuman, 1997; Katz, 1997). Navigating the dynamics of these synergies enables the EC and ECSE leaders to provide for systematic and sustainable transformation of the settings so that people working in these settings can practice their roles efficiently and effectively and better foster children's learning and wellbeing in general (Culkin, 2000; Kagan \& Hallmark, 2001; Katz, 1997).

\section{Statement of the Problem}

Despite the emerging body of research that confirms the pivotal role that EC and ECSE leaders play in quality education and care, research in EC and, specifically, in the field of ECSE has not kept pace with the changes that are occurring within the field (Bloom \& Bella, 2005; Bruns et al., 2017; Dennis \& O'Connor, 2013; Rohacek, Adams, 
$\&$ Kisker, 2010). Over the past 20 years, there have been different calls for leadership development in EC and very recently, in ECSE. Such calls aimed to emphasize the need to build and sustain leadership capital, human capacities, and organizational cultures to support active engagement in quality leadership (Bailey, 2000; Keilty, LaRocco, \& Casell, 2009). However, there is a paucity of research in the field to inform quality leadership development, implementation, and sustainability. Simply stated, there is little published literature on effective approaches for how to build, support, and sustain leadership capital across all domains of practice and levels of EC and, more specifically, in ECSE systems.

The goal of EC and ECSE programs is to assist both typically developing children and children with or at-risk for disabilities and their families to meet developmental and behavioral outcomes that will enhance their quality of life (Bruder, 2010). The service systems within which EC and ECSE professionals work are highly complex and are composed of multiple entities. They are administered by many different agencies (e.g., education, health, and human services), funded through numerous sources, and governed by multiple federal and state laws. Thus, effective leadership in EC and ECSE is crucial to quality service provision.

Some factors contributing to the lack of effective and efficient leadership development and sustainability in the field include: (a) the dearth of research in leadership in EC and, more specifically, in ECSE; (b) the fluid and evolving definition and lack of operationalized conceptualization of leadership in the field; and (c) the variations in state and federal requirements for leaders that result in the fields' lack of understanding of effective and efficient leadership development strategies. These factors 
also impact the field's capacity to build, nurture, and preserve leadership capital, improve the working knowledge of the field, and maintain an ethic through which leaders bring about improved outcomes for children and families. Such outcomes would ultimately result in increased consistency and less fragmentation across programs as well as greater sustainability of leadership programs.

The relative lack of research to investigate leadership activity in the field and, by association, the absence of quality leadership development programs would seem to be a major oversight given the growth and the importance of the EC and ECSE sectors. What the fields of EC and ECSE need is a heightened consciousness and a systematic awareness of what specific elements comprise and sustain leadership for effective and efficient operation of EC and ECSE programs.

The DEC position statement on leadership (2015) recommended three core ideas to guide the future steps toward leadership development in EC and ECSE. One of the suggested core ideas was the need for the field to collect evidence about the current construct and demonstration of leadership skills across EC and ECSE service systems. Using such evidence, leadership development in the field can be informed, and the knowledge base on effective leadership practices can be improved. Realization of existing leadership construct and practices is considered as a prerequisite for quality, data-informed leadership development in the field to better meet the arising needs of families and children with or at risk for developmental delays/disabilities who are the primary stakeholders and beneficiaries of the outcomes of effective leadership (DEC, 2015). Such realization might include: (a) the state of leadership implementation, (b) the extent to which the leadership practices align with the available evidence in the field, (c) 
the qualifications of people in charge of leadership roles and if their qualifications and preparation meet their job requirements, (d) the encountered challenges that negatively impact leaders' performance, and (e) the supports needed to further equip the leaders to be more effective. However, no study to date, either in the fields of EC in general or ECSE, has investigated leadership qualifications, the leadership implementation landscape, or the specific challenges that EC and ECSE leaders have and the supports these leaders need for quality leadership development. This study aimed to step forward by unraveling the implementation of current leadership practices and areas in need of further support in the field.

Goffin (2013) persuasively argued that we are experiencing a defining moment for EC and ECSE. Over the past few decades, the fields have grown dramatically in both public visibility and public scrutiny. But despite important advances, EC and ECSE lack clarity about their purposes, and boundaries and are still largely shaped by external policy forces. Goffin (2013) exhorted a call to action, stating that leadership is needed within the fields of EC and ECSE to transform them into coherent, competent, and accountable professions. Expanding the leadership capacity of each and every program is an important step in that direction. If the field aims to develop effective and efficient leadership, it needs to be based on a clear realization of how the leaders in the field are performing, what needs and challenges they have, and what kind of support they need to improve.

\section{Purpose of the Study}

The purpose of this study was to improve the understanding of the current leadership practices in the field and necessary required supports for leadership 
development and sustainability. This study aimed to examine leadership practices by district level and county level EC and ECSE leaders in the state of Colorado to identify: (a) who the leaders are in terms of their leadership qualifications and background, (b) the current state of leadership implementation, (c) the challenges and barriers that affect EC and ECSE leaders' performance, and (d) the support these leaders need to practice quality leadership development and sustainability in the field.

This study investigated district and county level EC and ECSE leaders who administer state and federally funded EC/ECSE programs. These leaders are the agents closer to practice and most often ignored when it comes to examination of the needs and challenges. Reducing the research-to-practice gap has been a burning topic in research during the past decade (Fixsen, Blase, Metz, \& Dyke, 2013). Implementation science emphasizes that thoughtful and effective implementation strategies at multiple levels are essential to any systematic attempt to use the products of science to improve the lives of children, families, and adults (Fixsen et al., 2013). This requires a clear understanding of the components and factors that promote effective implementation of evidence-based practices. According to the National Implementation Research Network (2019), the first stage of implementation analysis includes an exploration phase which deals with "where we are?" As such, evidence-based leadership development and sustainability can be regarded as a multi-step implementation procedure, starting with a data-informed exploration of where we are and what we further need for improvement.

This study had the potential to shed light on the leadership exploration phase, by investigating the existing leadership implementation trends, issues, and areas in need of support in the field and recommend initiatives that can improve quality leadership 
development and sustainability. It is necessary to unravel how leadership in the field is conceptualized, is being implemented, and by whom and under what challenges in order to guide quality, evidence-based leadership development. That way, improvements can be exercised at a more grassroots level, and appropriate interventions and strategies can be designed and tested to improve leadership capacity and sustainability in the field.

\section{Significance of the Study}

This study was considered significant for a number of reasons. First, the findings of this study may guide the fields of EC and ECSE to plan data-informed leadership development and professional development programs. Data-informed programs will have the capacity to build, nurture, and preserve leadership capital, improve the working knowledge of the field, and maintain an ethic of accountability that could result in increased consistency, less fragmentation, and greater sustainability across programs.

Second, this study can contribute to the understanding of leadership in EC and ECSE by producing scholarly research on a topic that is poorly researched. In particular, with the rapidly rising percentage of children with special needs in EC and ECSE services (National Center for Education Statistics [NCES], 2019), the quality of EC and ECSE programs becomes an even more significant issue. Along with the increasing demand for quality comes the increasing importance of the quality of leaders and quality leadership practices.

Third, EC and ECSE program leaders may benefit from this study by being encouraged to conduct a self-evaluation leadership study through either the use of the research methods that were used in this study or other leadership evaluation tools available in the field. Conducting an assessment on the current leadership implementation 
and identifying needs and supports for future leadership development is an opportunity for EC and ECSE leaders to make themselves aware of their own strengths and weaknesses as leaders and become more intentional in their practices. This consequently impacts EC and ECSE professionals, teachers, and service providers who may benefit from the satisfaction that comes from working in an organization that employs researchrecommended practices and collective intellect to improve programs' quality.

Fourth, the government agencies and policy makers, who are concerned with the quality of programing being delivered, either in accordance with the Individuals with Disabilities Education Act (IDEA) Part C or IDEA Part B, section 619 or other federal programs (e.g., Head Start/Early Head Start) may also benefit from this research by gaining insight into the existing leadership implementation status in the field and needs and concerns for future planning of effective leadership professional development in either federal or state-funded programs. Policy makers are accountable for funds that are designated for state-funded EC and ECSE programs. Therefore, they might be interested in seeing that funds are being directed and used to affirmatively impact program quality. Lastly, children and their families also may benefit from this study by receiving a better EC and ECSE experience in a program that is enhanced through better leadership. The ultimate reason for improving leadership is to improve programs' quality to better prepare children for success in their future education and life.

In summary, the findings of this research can identify more precisely how EC and ECSE leaders perform and, by association, what leadership professional development is required to maximize the effectiveness of all leaders. Considering the complexities of the EC and ECSE systems and the challenge of existing multi-disciplinary service provision, 
it is necessary for the future EC and ECSE research to guide preparation of skilled leaders in order to fulfil their roles and responsibilities effectively.

\section{Research Questions}

The following research questions guided this study:

Q1 Who leads district-level and county-level early childhood and early childhood special education programs? What leadership competencies and academic and professional background do they have?

Q2 How do district-level and county-level early childhood and early childhood special education leaders evaluate their leadership implementation?

Q3 What challenges do these leaders encounter in leadership implementation and development in their programs?

Q4 What professional development and support do these leaders need to implement quality leadership in their practice?

Q5 How do state-level leaders perceive the leadership implementation, challenges, and professional development needs of district-level and countylevel early childhood and early childhood special education leaders?

\section{Definition of Terms}

Child Find Colorado: Child find is part of Colorado's system for identifying children suspected of having a delay in development. The purpose of the evaluation is to determine if there is a significant delay or if there is a need for early intervention or special education services.

CPP: The Colorado Preschool Program (CPP), under Colorado Department of Education, was launched in 1988 in an effort to target 3- and 4-year-olds in need of language development support, with the goal of lowering school dropout rates and reducing dependence on public assistance.

Developmental Delay: A child with a developmental delay shall be 3 through 8 years of age and experiencing developmental delays in one or more of the following 
areas: physical, cognitive, communication, social or emotional, or adaptive, which prevents the child from receiving reasonable educational benefit from general education.

ECE: Early childhood education occurs from birth through age 8, including early learning from birth through age 2, preschool education from age 3 to age 5 , and kindergarten through third grade education that covers ages 5 to 8 .

ECSE: Early childhood special education services are provided to children, birth through 8 years of age, and their families. These young children have or are at risk for developmental delays/disabilities in one or more areas of development.

EI: Early intervention (EI) is a collection of services for eligible young children that exhibit developmental delays from birth through the child's third birthday.

EI Colorado: Early Intervention Colorado (EI Colorado), under the Office of Early Childhood, Colorado Department of Human Services, is Colorado's Lead Part C agency that is responsible for providing early intervention services under IDEA (2004) in the state of Colorado.

FAPE: Free and Appropriate Public Education (FAPE) is guaranteed by Public Law 94-142 and IDEA. Under the IDEA, FAPE is defined as an educational program that is individualized to a specific child, that meets that child's unique needs, provides access to the general curriculum, meets the grade-level standards established by the state, and from which the child receives educational benefit.

HS/EHS: Head Start/Early Head Start (HS/EHS) is a federal program that promotes the school readiness of children from birth to age 5 from low-income families by enhancing their cognitive, social, and emotional development. 
IDEA, Part B, Section 619: The Preschool Grants for Children with Disabilities program of IDEA, Section 619, supplements funding available for children ages 3 through 5 with disabilities under the Grants to states program of IDEA, Part B.

IDEA, Part C: Individuals with Disabilities Education Act (IDEA), Part C refers to the early intervention (EI) programs for infants and toddlers with disabilities as early as birth through age 2 and their families that help to improve child developmental outcomes that are critical to educational success.

IEP: Individualized Education Plan (IEP) is a written plan for the provision of appropriate special education services to an eligible child/student who has a disability identified under the law from preschool through age 21. It is based on and responsive to the child's/student's multi-disciplinary team evaluation. The IEP focuses on special educational and related services.

IFSP: Individualized Family Service Plan (IFSP) is designed for the child and family from birth through age 3 to include the outcomes important to the family, details about the services provided, and the recognition that goals and objectives for the family as a unit will be developed. An IFSP is based on an in-depth assessment of the child's needs and the needs and concerns of the family.

\section{Summary of the Chapter}

This chapter provided some general background knowledge and context for this research study. Leadership definition along with a conceptualization of leadership in EC and ECSE context was presented. Ecological System Theory was introduced as the theoretical framework, encompassing the structure that supported this study. The NAEYC's proposed model of leadership was discussed as the conceptual framework 
which shaped the operational definition of what is meant by "leadership" in this study. Significance and specific research questions were also presented. Next, in Chapter II, through a review of the literature, leadership in EC and ECSE along with leadership research trends and the existing gaps in need of further investigation will be discussed. The research design and method to carry out the study and the data analysis procedures will be presented in Chapter III. 


\section{CHAPTER II}

\section{REVIEW OF LITERATURE}

The purpose of this chapter is to review literature that is relevant to this study. The chapter begins with a brief overview of early childhood (EC) and early childhood special education (ECSE) services. Later, leadership is defined, and leadership theories and models in general and in the EC context are explored. An examination of leadership research trends and leadership issues and practices in EC and ECSE is also presented. The chapter concludes with identification of gaps in the literature and needs for further investigations in the field, particularly those that relate to this dissertation research.

\section{An Overview of Early Childhood and Early Childhood Special Education}

\section{Early Childhood Education}

The National Association for Education of Young Children (NAEYC) defined EC education as occurring from birth through age 8 , including early learning from birth through age 2, preschool education from age 3 to 5 and kindergarten through third grade education that covers ages 5 to 8 . According to the NAEYC, it is during this period that a child goes through the most rapid phase of growth and development and gets prepared for later education (National Government Association, 2019).

In terms of birth through age 5 (pre-kindergarten), EC education can include: (a) any state-licensed or state-regulated program or provider, regardless of the setting or 
funding source, that provides early care and education for children from birth to kindergarten entry, including, but not limited to, programs operated by child care centers and in family child care homes; (b) preschool programs funded by the federal government, state or local educational agencies (including IDEA-funded programs); (c) Early Head Start and Head Start programs (EHS/HS); and (d) any non-relative child care providers not otherwise regulated by the state for two or more unrelated children for a fee in a provider setting (U.S. Department of Education, 2019a). In recent years, EC education has become a prevalent public policy issue, as local, state, and federal lawmakers consider funding for preschool and kindergarten programs. In the past decade, there has been a national push for state and federal policies to address the early years as a key component of public education (U.S. Department of Education, 2019b).

The first years of life are critical for later outcomes. Young children have an innate desire to learn. That desire can be supported or undermined by early experiences. High-quality EC education can promote intellectual, language, physical, social, and emotional development, creating school readiness and building a foundation for later academic and social competence. The EC education programs have the potential to help equalize that children have the chance to learn, preparing all children for success in the classroom and beyond and having a particularly powerful impact on children from lowincome households. A wide body of rigorous research has found this to be true. The Learning Policy Institute (Meloy, Gardner, \& Darling-Hammond, 2019) conducted a new study that pulled together findings from the most rigorous contemporary evaluations, concluding the great weight of evidence showing that rich, engaging EC/ECSE programs make a substantial difference in preparing children for school and can help mitigate 
developmental gaps. The study found that children who attend quality EC education experience substantial learning gains and are more prepared for school than children who do not attend EC education. The research also shows investments in EC education bolster student success and have positive impacts on children's early literacy, math, and, in many cases, social-emotional skills.

One of the key factors contributing to differential outcomes, according to the research by the Learning Policy Institute (Meloy et al., 2019), includes the quality of the EC program. EC/ECSE programs that demonstrated the strongest and most persistent positive effects in terms of achievement, school progress, and educational attainment invested in the essential building blocks of high-quality EC education, including teacher preparation, thoughtful curriculum, meaningful family engagement, and support for English learners and students with special needs which are byproducts of effective and efficient leadership. The EC/ECSE education programs that sustain these benefits provide students with ongoing academic challenges that build on the child's early learning. According to the report by the Learning Policy Institute (Meloy et al., 2019), the evidence is clear. It's time to move beyond the question of whether EC/ECSE education "works" and focus, instead, on the more pressing question of how to design and implement quality programs that ensure EC education investments consistently deliver on their promise. Quality doesn't just happen. We must design it, support it, and continually work for it.

\section{Early Childhood Special Education}

Individuals with Disabilities Education Act (IDEA), Part C. The Education of the Handicapped Act Amendments of 1986 established the Early Intervention (EI) 
Program for infants and toddlers with disabilities under Part H (now Part C) of the IDEA. Providing EI services to children with disabilities as early as birth through the child's third birthday and their families helps to improve child developmental outcomes that are critical to educational success. Early intervention services are designed to identify and meet children's needs in five developmental areas: physical development, cognitive development, communication development, social or emotional development, and adaptive development. The EI program assists states in developing and implementing a statewide, comprehensive, coordinated, and multidisciplinary interagency system to make EI services available for all infants and toddlers with disabilities and their families (U.S. Department of Education, 2018).

\section{Individuals with Disabilities Education Act (IDEA), Part B, Section 619.}

Under Part B of the IDEA, funds are provided to states to assist them in providing a free appropriate public education (FAPE) to children ages 3 through 21 with disabilities who are in need of special education and related services. The Preschool Grants for Children with Disabilities Program of IDEA, Section 619, supplements funding available for children ages 3 through 5 with disabilities, under the Grants to States Program of the IDEA, Section 611. To be eligible for funding under the Preschool Grants for Children with Disabilities program and the Grants to States Program for children ages 3 through 5, a state must: (a) ensure that all children with disabilities have FAPE available to them and receive special education and related services designed to meet their individual needs; (b) ensure that the rights of children with disabilities and their parents are protected; (c) assist states and localities to provide for the education of all children with 
disabilities; and (d) assess and ensure the effectiveness of efforts to educate children with disabilities (U.S. Department of Education, 2018).

States report data annually to the Office of Special Education Programs (OSEP) in the U.S. Department of Education on three child outcomes for Part C and Part B Preschool programs: (a) social relationships, which includes getting along with other children and relating well with adults; (b) use of knowledge and skills, which refers to thinking, reasoning, problem-solving, and early literacy and math skills; and (c) taking action to meet needs, which includes feeding, dressing, self-care, and following rules related to health and safety. Using the three child outcomes categories, states report annually to OSEP the percentage of children who: (a) did not improve functioning; (b) improved in functioning, no change in trajectory; (c) moved closer to functioning like same-aged peers; (d) improved functioning to that of same-aged peers; (e) and, functioning like same-aged peers (U.S. Department of Education, 2018).

According to the Early Childhood Technical Assistance Center and Center for IDEA Early Childhood Data Systems' report (2019), the data show that large percentages of children continue to show greater than expected gains in both IDEA Part C and Part B, Section 619 services, and large percentages of children continue to leave the programs with age-expected skills. There has been little year-to-year change in the numbers, which speaks to the stability of the data (U.S. Department of Education, 2018).

\section{Leadership in Early Childhood and Early Childhood Special Education}

A conceptualization of leadership in EC and ECSE context was presented in Chapter I. The NAEYC's proposed model of leadership was discussed both as the best fit for EC settings and identified as the operational definition of leadership in this study. In 
what follows, leadership is defined based on current literature, and different leadership theories and models are provided. The Whole Leadership Framework that is drawn from NAYEYC's proposed model is also discussed.

\section{Leadership Defined}

Leadership often means different things to different people and is defined based on the specific settings and environments where leadership takes place (Sullivan, 2010). Whereas numerous definitions of educational leadership exist, one that appears to capture the central meaning of leadership is provided by Robbins, Millet, Cacciope, and Waters (1998) who said leadership is "the ability to influence others towards the achievement of goals that contribute to a worthwhile purpose" (p. 396). Fullan (2001) saw the new meaning of leadership as what makes "each and every educator strive to be an effective change agent" (p. 13) and Truskie (2002) asserted "there is a direct link between leadership, organizational culture and performance" (p. 1).

Pace (2002) also articulated that leadership is such an important part of any workplace that the workplace "cannot be understood clearly without understanding the function of leadership in the system" (p. 33). Fullan (2001, p. 261) also agreed when he wrote: "Nowhere is the focus on the human element more prevalent than in the recent recognition of the importance of strong and effective leadership." He later added: "Effective school leaders are the key to large-scale, sustainable education reform" (Fullan, 2004, p. 15).

There are other definitions for leadership, specific to EC/ECSE, provided by professional organizations such as the Division for Early Childhood (DEC) of the Council for Exceptional Children (CEC). The DEC is the largest organization that 
promotes policies and advances evidence-based practices that support families and enhance the optimal development of young children (ages 0-8) who have or are at risk for developmental delays and disabilities. The DEC (2014) defined leaders as "those in position of program authority and leadership related to providing services to all young children who have or are at risk for developmental delays/disabilities and their families" (p. 6). Examples of such leaders include state, regional, and local administrators, early childhood coordinators, building principals, assistant directors, and coordinators. In March, 2015, the DEC developed a position statement on leadership to guide promoting high-quality leadership at all levels of the EC and ECSE service systems. According to the DEC position statement (2015), leadership is defined as "the proactive process of influencing others to act for certain goals that represent the values and motivations, the wants and needs, aspirations and expectations of both leaders and followers" (Burns, 1978, p. 19). While the field of EC and ECSE does not have a consensus definition of leadership, conceptualization of leadership from other fields informs the field's thinking (Bolman \& Deal, 2013; Yukl, 2013).

\section{Leadership Vs. Management}

Cuban (1988) provided a clear distinction between leadership and management. He linked leadership with change and saw management as a maintenance activity. According to Cuban (1988), leadership includes influencing others' actions in achieving desirable ends. Leaders are people who shape the goals, motivations, and actions of others. Management is more about maintaining organizational arrangements. While managing well often requires leadership skills, the overall function is toward maintenance rather than change. 
Understanding and distinguishing between leadership and management is essential. In order to lead EC and ECSE programs effectively, leaders need to recognize that they are actively promoting change and improvement to work with and provide support to other people. Early childhood and ECSE leaders need to be able to both lead and manage as well as recognize when each is appropriate. For Cuban (1988), leadership meant fostering changes that improve EC/ECSE programs. Thornton and Cherrington (2014) suggested that much of the difficulty in understanding leadership in EC can be attributed to confusion about the differences between leadership and management. Leadership defined through this perspective leaves out the possibility for other leaders to emerge, such as parents and the EC educators who work directly with children. The differences between leaders and managers has also been acknowledged by the DEC position statement on leadership (DEC, 2015).

\section{Leadership Theories and Models}

\section{Classical Leadership Theories}

Theorists, researchers, and practitioners have spent decades researching and analyzing the subject of leadership that laid the groundwork for development of contemporary leadership models (Fullan, 2004). These classical theories may be placed into one of three categories: trait theories, behavior theories, or situational leadership theories. The following section outlines a summary of the classical theories of leadership development.

Trait theories. Trait theories of leadership assume that people are born with inherent traits and characteristics that make them leaders. Some traits are viewed as particularly suited to leadership, and people who make good leaders are viewed to have 
the right combination of these traits (e.g., vision, integrity, and passion). Today, it is believed that a single set of traits are not likely to prove to be effective in all situations, and that a leader who is successful in one setting may not be successful in another (Green, 2005).

Behavior theories. Behavior theories of leadership do not search for inherent traits or characteristics, but rather, they look at a leader's behavior. Furthermore, because behavior theories focus on the action of the leader, these theories assume that leadership skills can be developed. Behavior theories focus specifically on the manner in which goals are established, roles and task requirements are clarified, and leaders motivate followers in the direction of goal attainment (Daresh, 2002).

Situational leadership theories. Situational theories of leadership focus not only on the leader, but on the problem identification and decision-making processes in different settings. More specifically, they focus on three factors and their interactions: the leader, the follower, and the situation (Daresh, 2002). In situational leadership the focus is not on "the right approach" but rather an approach that takes into account the followers and the situation (Green, 2005).

\section{Contemporary Leadership Models and Dimensions}

As mentioned earlier, the classical theories of leadership laid the groundwork for today's contemporary leadership models, and the findings have informed leadership practices of many educational leaders (Grint, 2010). Considering the contemporary leadership models, Grint (2010) identified four areas of consistent dialogue in regard to classifying leadership that include leadership as a position, as a person, as a result, and as a process. According to Grint (2010), viewing leadership through these four lenses can 
help in our understanding of leadership. In the sections below, leadership through these four perspectives is explored.

Leadership as a position. The idea most prevalent in the literature on leadership is that leadership and management mean one and the same. Research framed through this leadership lens focuses preparing individuals for management positions to lead and manage programs and people (e.g., Bloom \& Sheerer, 1992; Fleming \& Love, 2003; Hayden, 1997; Isles-Buck \& Newstead, 2003; Rodd, 1997). Position-based leadership implies a hierarchy, with someone "above" driving change from the top through “subordinates" who follow the leader's demands (Grint, 2010). This form of leadership resembles the role of management and supervision because it requires followers to surrender, or hand over power, to these positions. If leaders act based on position, then according to Grint (2010), only those in formal positions of power will be recognized as leaders.

Leadership as a person. Leaders are often identified as those with titles. They are viewed as being ahead of followers and are presented as being somehow more intelligent, experienced, individual, controlling, determined, and focused (Bella, 2013; Bloom, 2014; Bloom \& Bella, 2005; Preskil \& Brookfield, 2009; Talan et al., 2014). In this leadership model, the individual is often held to a higher standard and viewed as what Grint (2010) called the "heroic" leader who is responsible for delivering leadership and who has specific characteristics or a charismatic personality.

Leadership as a result. This leadership model requires strong leaders to ensure that investments are spent on necessary resources to meet predetermined goals and outcomes. Grint (2010) suggested that this form of leadership may be perceived as "the 
purpose of leadership" (p. 8), with a focus on results being a criterion for leadership as well as an attribute of a leader. Viewing leadership through this lens is strongly associated with the traditional definition of leadership that highlights specific skills, attributes, and roles and suggests that one individual is directly responsible for outcomes, either positive or negative (Grint, 2010).

Leadership as a process. This model of leadership is what was discussed as the NAEYC's proposed leadership model as the best fit for EC settings in Chapter I of this study. Supported by Kagan and Bowman (1997), Kagan and Hallmark (2001), and Taba et al (1999) as the best fitting model for EC contexts, leadership as a process identifies the same five "faces" of leadership: administrative, pedagogical, community, conceptual, and advocacy. For a more detailed discussion of each of the five faces, please refer to Chapter I.

Drawing from the NAEYC's proposed model, the McCormick Center for Early Childhood Leadership developed the Whole Leadership Framework (Abel et al., 2016). Although the classification of the five leadership faces of the NAEYC's proposed model (administrative, pedagogical, community, conceptual, and advocacy) differs with the classification in the Whole Leadership Framework, the core ideas of both models convey the same attributes. Below, a description of the Whole Leadership Framework and how it aligns with the NAEYC's proposed model is discussed. In both the Whole Leadership Framework and the NAEYC's proposed model, there is an overlap between each of the domains or faces, reflecting an interdependent relationship. Few leadership roles and functions are mutually exclusive. Rather, leadership exercised in one domain impacts and/or requires reciprocal leadership in the other domains (Abel et al., 2016). 


\section{Whole Leadership Framework in Early \\ Childhood Education}

The Whole Leadership Framework highlights three primary domains including:

(a) administrative leadership that includes operational, strategic, advocacy, and community elements of leadership; (b) pedagogical leadership that includes instructional leadership and family engagement; and (c) leadership essentials which is a representation of conceptual leadership elements in the NAEYC's proposed model. Below, the three domains of the Whole Leadership Framework are discussed.

\section{Whole Leadership Framework:} Administrative Leadership

In the Whole Leadership Framework, administrative leadership includes

operational, strategic, advocacy, and community elements of leadership. It is about setting goals, orchestrating work, and mobilizing people to sustain an EC organization. Effective administrative leaders establish systems for consistent implementation of program operations to meet the needs of children, families, and staff (Culkin, 1997). Administrative leadership requires involvement in the day-to-day operation of EC services, including supervision, budgeting, and issues related to human resources (Culkin, 2000). Much of the literature focuses either on preparing managers to perform administrative and supervisory tasks or on personal attributes that leaders are assumed to possess or need (e.g., Bloom \& Sheerer, 1992; Hayden, 1997; Kagan \& Bowman, 1997; Neugebauer \& Neugebauer, 2003; Rodd, 1997; Thornton \& Cherrington, 2014). The EC director's primary goal is considered to be operational: to deliver quality EC service and support staff development. According to Woodrow and Busch (2008), this model implies that leadership is something exceptional rather than an everyday practice. The assumption 
that leadership is connected to management has led to some EC educators and workforce (those working directly with children) resisting possibilities to see themselves as leaders. In the Whole Leadership Framework, administrative leadership is composed of four subdomains including: operational, strategic, community, and advocacy leadership.

Operational leadership is accomplished through critical functions such as hiring, evaluating, and supporting teaching staff. Additional critical functions of operational leadership include developing budgets aligned with program goals and needs and maintaining a positive organizational culture and climate (Abel et al., 2016; Culkin, 1997, 2000).

Strategic leadership involves guiding the direction of EC organizations with the future in mind. Strategic leaders clarify mission and values, inspire staff to pursue a shared vision, and ensure that program goals and outcomes are attained. Strategic leaders need to communicate and align their program services with those of other community organizations serving young children and families (Abel et al., 2016; Culkin, 1997, 2000).

Community leadership involves demonstrating to the community that $\mathrm{EC}$ is an important issue by: being visible in the community; working to develop community partnerships; engaging in dialogue and conversation in different formal and informal community settings; listening to what the various stakeholders hope for children, families, and the EC sector; and becoming involved in educating future workers in the EC sector. To further involve the community, the EC programs partner with EC leaders to facilitate their collaboration and seek their input regarding who should be invited to the table for the consultations (Crompton, 1997). 
Advocacy leadership is another essential aspect of strategic leadership. Effective administrative leaders are future oriented and engage in advocacy because they want to have influence on the external conditions (professional standards, regulations, and government policies) that impact young children, families, and EC programs (Blank, 1997). Advocacy leadership requires strong leadership and willingness to act to help improve the landscape for children, families, and the EC sector. Issues such as regulations, workforce disparity, affordable and accessible quality child care, and other social equity issues are addressed by working with people and organizations beyond the immediate sector/environment to inform policy change (Blank, 1997).

\section{Whole Leadership Framework: Pedagogical Leadership}

Pedagogical leadership aims to improve the art and science of teaching. Pedagogical leadership attends to educator dispositions and high-quality interactions with children. Activities of individuals exercising pedagogical leadership include ensuring fidelity to curricular philosophy, assessing children's development and learning, using data for evaluation, and optimizing learning (Muijs et al., 2004). Effective pedagogical leadership requires promoting partnerships with families, which is essential to children's learning and growth. Therefore, depending on the setting and organizational structure, pedagogical leadership in EC programs may also foster families (Heikka \& Waniganayake, 2011). Pedagogical leadership has gained momentum and has been more widely referred to in the EC sector today in many countries (Aubrey, Godfrey, \& Harris, 2013; Fonsen, 2013; Heikka \& Waniganayake, 2011). This approach to leadership acknowledges the strengths, experiences, and skills held by those in the field and 
encourages them to share their knowledge with peers and colleagues (Sims, Forrest, Semann, \& Slattery, 2015).

Quality pedagogical leadership requires strong administrative leadership. The most qualified, skilled teachers will not be effective if the organizational conditions in which they work (e.g., supervisor support, opportunities for professional growth, shared decision-making, collegiality, role clarity, task orientation, innovativeness, and physical environment) are not adequately supportive (Heikka, Waniganayake, \& Hujala, 2013).

\section{Whole Leadership Framework: Leadership Essentials}

Leadership essentials include the personal skills and attributes on which administrative and pedagogical leadership are built (Abel et al., 2016). Leadership essentials are embodied by the leader as necessary anchors for success. Leadership essentials create the fertile and healthy environment that results in a greater degree of collaboration, efficacy, creativity, and ethical commitment for everyone involved, including the leader. Leadership essentials revolve around the creation of new "concepts" and ideas to advance the EC profession (Abel et al., 2016). This is regarded as “conceptual leadership" in the NAEYC's proposed model of leadership in EC settings.

Conceptual leaders are open to new ways and thinking and are prepared to think forward. Conceptual leadership focuses on the need for critical and reflective thinking, collaborative and ongoing learning, building relationships, and viewing EC leadership different from mainstream management perspectives (Kagan \& Neuman, 1997). Engaging in dialogue and reflective thinking is a way that EC directors can be present and curious in efforts to create a process of learning and knowing by exploring their lived 
experiences, and after doing so, they can then deal critically with that process (Aubrey et al., 2013; Muijs et al., 2004).

\section{Other Leadership Models in Early Childhood}

Contextual leadership, collaborative leadership, adaptive leadership, and distributed leadership are among other leadership models that have been studied by some EC researchers. Below, a brief synopsis of these studies in terms of each model is presented.

\section{Contextual Leadership Model}

In terms of contextual leadership, according to Bloom (1998), one key essential element inherent is the movement from beginning leader to master leader. Bloom (1998) described the movement as "far more complex, however, than the mere accumulation of new knowledge and acquiring skills" (p. 8). Bloom's study (1998) also showed that the context and circumstances surrounding a leader's growth and development, including the environment, relationships, and external situations, play a role as individuals advance in their position. Bloom (1998) contended that directors move in and out of career stages in response to personal and environmental factors. Bloom (1998) suggested that for adults to grow and develop, they must create contexts that encourage and celebrate their development.

Hujala (2004) also investigated EC leadership using a contextual leadership model, examining the leadership context from people involved with the leadership. Focus groups were conducted with discussion topics concerning: the definition of leadership; the challenges, roles, and responsibilities of leadership; leadership tasks; and the quality of EC education connected to leadership. One key finding essential to the contextual 
leadership of the Hujala (2004) study model was that the leadership context defined the leadership culture. This study offered a unique opportunity to see what leadership looks like from a range of perspectives. The values and language of leadership were found to differ based on the perspective of the leaders who examined leadership issues in terms of their own viewpoint (Hujala, 2004).

\section{Collaborative Leadership Model}

According to Aubrey (2007) one key that is essential to the collaborative leadership model is that the most successful early childhood leaders are those who widened the boundaries of leadership, creating a work culture that is participative and give their staff the opportunity to exert "bottom-up" influences on their own professional practice. Aubrey (2007) suggested that this model of leadership was viewed as effective because it acknowledged the wide range of skills available within the workplace and distributed responsibility accordingly. This form of leadership was also reported to be empowering, as it centered on a model of leadership that recognized that capabilities and expertise are distributed or shared among many (Aubrey, 2007). In terms of leadership development, Aubrey (2007) suggested that if a collaborative leadership model is going to be used within EC settings, then all EC professionals would need to have the skills necessary for teamwork and such as collaboration, communication, and decision making.

Kagan and Hallmark (2001) focused their research on three separate dimensions of collaborative leadership including: (a) identifying leadership, (b) implementing leadership, and (c) sharing leadership. One key finding essential to the collaborative leadership model in their research was that identifying the leader was not a straightforward task in six of the eight collaborations. All of the collaborations were 
headed by a person who carried the "official" administrative title and, thus, leadership was dispersed among many. Yet, while sharing the leadership role is a chief component of the collaborative model, Kagan and Hallmark (2001) found that this concept is not as simple as stating that shared leadership exists or it does not exist. Rather, a continuum of leadership existed across the programs studied, beginning with sole leadership, then supported leadership, and finally shared leadership at the other end of the continuum. Over the years, collaborative leadership has evolved into newer terms as shared leadership or distributed leadership. Distributed leadership is more commonly used as the current trend.

\section{Distributed Leadership Model}

Building leadership capacity in EC and ECSE context requires a shift from thinking about a model of leadership in which authority resides in a single heroic figure to thinking about a model of distributed leadership in which leadership is practiced by many staff at a program, who share responsibility and accountability (Goffin, 2013). In this broader view, leadership in EC and ECSE is viewed as a fluid organizational asset that is not fixed but, instead, can be strengthened and expanded (Talan, 2010).

Distributed leadership research is relatively young, emerging as a focus of research during the late 1990s and is primarily concerned with the study of school-based leaders (Talan, 2010). In terms of EC leadership, Ebbeck and Waniganayake (2003) introduced the concept of distributed leadership, and others such as Aubrey (2007) have endorsed its exploration within EC settings. Distributed leadership is a hot topic in the educational leadership world. Much has been written about taking a distributed approach to leadership in K-12 schools, and there is recent support for the efficacy and efficiency 
of this model of leadership to help program administrators improve their leadership practice in EC settings (Heikka et al., 2013; Muijs et al., 2004; Talan, 2010).

What is new and exciting about distributed leadership is that it puts leadership practice front and center. Most of what has been written on the topic of leadership is actually focused on leaders and their traits and characteristics (Heikka et al., 2013). The vast majority of leadership literature quickly moves from the study of leadership to an examination of leaders where leadership is understood as leaders who are bold and dominant, charismatic and influential, or visionary and transformational (Talan \& Bloom, 2011). A distributed approach to leadership widens the lens to focus attention equally on leaders, followers, and specific situations. This broader perspective allows us to see the interdependencies between all three, which is the actual practice of leadership (Talan, 2010).

Heikka et al. (2013) conducted a thorough literature review to establish a research agenda on distributed leadership in EC. The review indicated that distributed leadership approaches can assist in the implementation of leadership responsibilities by bringing about better interconnection, consistency, and coherence in service delivery among diverse stakeholders. In addition, the importance of developing closer connections with families and communities in EC organizations should reflect the necessity to explore collaborative ways of enacting leadership within contemporary educational settings. Accordingly, the theoretical roots of distributed leadership could inform future leadership studies undertaken within both school and EC education organizations (Heikka et al., 2013). 


\section{Application of Leadership Concepts to Early Childhood and Early Childhood Special Education}

For the field of EC and ECSE, the classic theories of leadership provide greater understanding of the nature of leadership, but do not give a full picture of the complexities of leadership and leadership development. There has been some research, as reviewed so far, conducted specific to EC settings that is based on more contemporary models of leadership such as contextual, collaborative, and distributed leadership. However, with the vast knowledge and research that has been completed in educational and business contexts, much should be learned from the generic leadership studies in other fields, and more should be done for the fields of EC and ECSE. The fields of EC and ECSE have several characteristics that, singly and in combination, make leadership development a more difficult task. Narrow definitions of leadership and lack of consensus around the definition in the field, ambivalence and attitude about authority that permeates the field, lack of systems thinking and a holistic view of the field, scarce resources and inappropriate competition for such scarcity, and narrow leadership preparation are among the significant difficulties.

Of all the difficulties mentioned above, lack of quality preparation is an influential factor in impeding leadership development and sustainability in the field. We, as a field, have not always encouraged leaders to seek wider domains or take on diverse roles. The need for developing a more extensive knowledge base and a theoretical framework concerning leadership roles and competencies in the field is essential. Emphasizing the importance of quality professional development and preparation, the following section provides information about the existing EC leadership preparation programs in the nation. 


\section{Current Status of Early Childhood Leadership Preparation}

The Licensing Education Analytic Database (L.E.A.D.) Early Childhood Clearinghouse (ECC) was launched in 2017 in response to the need for better data on the early childhood leadership workforce (Abel, Talan, \& Magid, 2018). The L.E.A.D ECC presents national and state statistics related to EC administration and leadership programs (site-based directors, family child care providers, and school principals), and the qualifications related to their education, professional development, and experience.

\section{National Profile}

The field of EC education has not defined national standards for the various roles practitioners play in the workforce. While the field is moving towards an acceptance of the bachelor's degree in EC education as the standard for lead teachers, there is less agreement about the requisite competencies and education of program leaders. Instead, federal and state regulatory systems have created a wide array of standards for individuals leading programs for children, birth through age 8. Also, professional preparation standards for elementary school principals are consistently more robust than those for EC program directors (Abel et al., 2018).

According to the L.E.A.D ECC, there are over 250,000 EC administrators in the United States distributed as follows (Abel et al., 2018):

- Family child care providers: 136,241

- Early childhood program directors: 61,800

- Elementary school principals: 75,760 


\section{Administrator Credentials}

The L.E.A.D ECC identified a total of 40 credential programs for EC program administrators, in 31 states and the District of Columbia, from the national scan in 2018. Nineteen states did not offer a credential. Also, there are three national director credentials available. One half of the EC administrator credential programs are tiered, based on criteria such as: general education specialized college credit or professional development in EC education; specialized college credit or professional development in administration, management, or leadership; and varied lengths of experience. Nine programs $(23 \%)$ require a minimum of an associate degree to be eligible for a credential. Twenty-five programs (63\%) require college credit hours in EC education in at least one level of the credential. Twenty-three programs (58\%) require college credit hours in administration, management, or leadership in at least one level of the credential (Abel et al., 2018).

\section{Leadership Preparation Programs}

Of the 3,065 EC degree programs in the United States, 100 programs (3\%) in 37 states, have a focus on EC management, administration, leadership, or advocacy. A majority of them (68\%) are offered at public institutions, with $45 \%$ at two-year colleges and 54\% at four-year institutions. Most programs (75\%) are delivered online, and 15\% are hybrid programs. Elementary principal preparation is offered in all 50 states and the District of Columbia through 1,479 programs by 777 institutions. Of these, four programs $(<1 \%)$ are for associate degrees, 154 programs (10\%) are for bachelor's degrees, 990 programs (67\%) are for master's degrees, and 334 programs (23\%) lead to a doctoral degree. The capacity of higher education to prepare elementary principals for the 
workforce is exponentially greater than that of preparing EC program leaders (Abel et al., 2018).

\section{Leadership Academies}

In addition to formal education for EC administrators, there are 32 leadership development programs, in 17 states, with a specific focus on EC program leadership. These leadership academies address various needs of program site directors including one or more of the domains of Whole Leadership Framework (pedagogical leadership, administrative leadership, and leadership essentials). The delivery approaches differ and may include coaching and mentoring, train-the-trainer options, online or hybrid learning, or face-to-face workshops (Abel et al., 2018).

\section{Head Start/Early Head Start}

Head Start and Early Head Start programs are regulated by the U.S. Department of Health and Human Services, Administration for Children and Families. An Early Head Start or Head Start Director is minimally required to have a bachelor's degree and experience in supervision of staff, fiscal management, and administration. However, the director may not necessarily be the site administrator of a program. Frequently, the director is located at the grantee or delegate agency level with oversight of multiple Head Start/Early Head Start sites (Abel et al., 2018).

\section{Suggested Implications for Future Research and Practice by the Licensing Education Analytic Database}

Advancements in standards for administrators of child care programs, primarily seen in voluntary state QRIS and state-funded pre-K, have not led to substantial improvements in the basic qualifications of most early childhood program administrators. 
However, the growing number of higher education degree programs in EC administration and specialized EC education leadership academies is an encouraging development (Abel et al., 2018).

A deep examination of the EC program leadership workforce and related state policies confirmed the assumptions that broad differences exist across states and sectors. With increasing evidence of the importance of EC education and the call for better educated and highly skilled EC teachers, there has been an astonishing lack of comparable calls for well-qualified and highly skilled site-based leaders of EC programs (Abel et al., 2018).

The L.E.A.D ECC emphasizes that across sectors, there is a pressing need for a unifying foundation of leadership qualifications and competencies reflecting a Whole Leadership Framework approach. A competent EC program leader needs knowledge and skills in child development, EC pedagogy, leadership essentials, and program administration (Abel et al., 2018).

The 2018 L.E.A.D ECC update also reaffirmed the challenges related to accessing consistent and comparable data on EC leadership. Segmentation in the field continues to create gaps in our knowledge about the EC leadership workforce. This gap is more apparent when it comes to the ECSE leadership workforce. Data are insufficient to help us understand the needs and challenges of the leadership workforce and to address leadership support services in areas that surround EC education. The L.E.A.D ECC encourages the development of more detailed state-level workforce registries to advance initiatives designed to support the EC workforce (Abel et al., 2018). 
The primary focus of what has been gathered and reported by the L.E.A.D ECC is mainly centered around EC education and leadership in general. However, the interconnectedness of the fields of EC and ECSE and inclusion movements that advocate for the full integration of children with disabilities in general education settings create a meaningful link between the suggestions put forward by the L.E.A.D ECC and the needs in the leadership domain in the field of ECSE. Considering the more limited resources, research, and initiatives regarding leadership in ECSE, the enumerated areas in need of a more unifying foundation of leadership competencies and more targeted efforts to work on leadership development and sustainability becomes more essential. This highlights the more critical role of current research to further the dialogue around intentional understanding and application of leadership in the field. Leadership literature in EC and ECSE is growing. However, leadership research in EC and specifically in the field of ECSE is still relatively limited. In what follows, leadership research trends in EC and ECSE is discussed and implications for future research is presented.

\section{Leadership Research Trends in Early Childhood and Early Childhood Special Education}

Despite the emerging body of research that confirms the pivotal leadership role EC and ECSE leaders play in quality education and care, research in this domain has not kept pace with the changes that are occurring within the field (Bloom \& Bella, 2005;

Bruns et al., 2017; Dennis \& O'Connor, 2013; Rohacek et al., 2010). Muijs et al. (2004) discussed the lack of importance given to leadership by EC workers and argued that the EC leadership research is limited and dominated by a relatively small number of researchers (e.g., Bloom, 1992, 1997, 2014; Fullan, 2000, 2001, 2004; Rodd, 1997, 2001, 2005). Since then, work by Aubrey (2007), Whalley, Wilson, and Allen (2008), and 
Gasper (2010), along with the literature review by Dunlop (2008), has begun to address this issue. Studies of the quality of EC settings identified effective leadership as important in raising quality and enhancing the working environment (Rodd, 2005). Aubrey (2007) also argued that much of the leadership research is not well informed by theory and research in the broader field of leadership studies. Theorizing, where it happens is limited and does not connect to key concepts in the educational, public sector, or business leadership. Kagan and Hallmark (2001) suggested that this may be because business leadership theories do not work well in EC education, which is a position shared with many professionals involved in education in other sectors. The authors suggested that a distinctive collaborative approach to leadership in EC is required.

Interestingly, the literature on EC and ECSE leadership does not connect with that on school leadership. This may be because of sector differences or the complexity of the field of EC and ECSE education, which is characterized by a greater diversity of organizations and institutions than the school sector (Aubrey, 2007). However, despite recognition that leadership is a key element of quality in early years' settings (Muijs et al., 2004), there have been few relevant empirical studies and paucity of the evidencebase in relation to the specific context of EC and ECSE service provision. What comes through most sources is that there is a high potential for leadership activity in the field of EC, but who provides quality leadership, and agreement about who might do so in EC services in the future is still more elusive and under-researched (Dunlop, 2008). According to Vandell and Wolfe (2000), there is ample research cited to support the 
claim that the higher the quality of early childcare and education, the greater the contribution to positive learning outcomes for children.

Both nationally and internationally, leadership research in EC is grounded in general EC education more than ECSE. In the United States, unlike organizations and coalitions involved with general EC education, such as the NAEYC or the McComick Center for Early Childhood Leadership at National Louis University which have published a good deal of resources to guide general EC educators and directors, there is a dearth in research that addresses leadership specifically in ECSE.

To identify and examine the available empirical literature on leadership in EI/ECSE, using various combinations of descriptors (e.g., Part C, EI, ECSE, and leader) an exhaustive search was conducted by the DEC in 2015 that included several electronic databases between 1985-2014 and resulted in few resources. As a second step, the three EI/ECSE peer-reviewed journals, including Infants and Young Children, Journal of Early Intervention, and Topics in Early Childhood Special Education, were also investigated and 33 articles that had the term leader or its derivatives (leaders, leading, or leadership) in the abstract were uncovered. According to the DEC (2015), half of identified articles failed to address matters of leadership in EI and ECSE, and the remaining articles were largely descriptive in nature with many combining the concept of management and leadership. Only six empirical investigations focusing solely on leadership in EI and ECSE were identified, and they had multiple and varied foci (e.g., Bays \& Crockett, 2007; Bruns et al., 2017; Epley et al., 2010; Sopko \& LaRocco, 2018; Summers et al., 2005). Recent and relevant research driven by calls from the field of ECSE was conducted by Bruns et al. (2017) that investigated necessary leadership competencies for 
effective ECSE leadership. While this body of work is somewhat informative, the wideranging emphases of these works and the paucity of large empirical investigations of leadership in EI and ECSE are quite limiting (DEC, 2015).

\section{A Systematic Review of Leadership Research Trends in Early Childhood and Early Childhood Special Education: A Brief Report}

Considering the limitations mentioned above in terms of leadership research in EC and more specifically in ECSE and the current vague understanding of conducted leadership research in EC and ECSE, a systematic review was conducted by Movahedazarhouligh (2019) aiming to fill the gap to some extent. The main objective of the conducted systematic review was to identify trends and landscape of the leadership studies in EC and ECSE that addressed birth through 8 years of age and identified areas in need of further investigation in the leadership domain in the field. A total of 124 studies that met the inclusion criteria were identified and summarized using a number of variables.

One of the areas of investigation in the systematic review was to identify how the leadership research trends differed between the fields of EC and ECSE. According to the findings, of 124 identified studies, 111 studies (89.6\%) addressed the field of EC in general and only 13 studies (10.4\%) focused specifically on the topic of leadership in ECSE. Another research question aimed to investigate leadership research themes that were studied in the fields of EC and ECSE specifically. Out of 111 studies in the field of EC, leadership roles were the most studied theme $(n=20,18.1 \%)$. Of the 13 studies within the field of ECSE specifically, inclusion was addressed by 3 studies (23.1\%) as the most studied theme. One other area of investigation in the systematic review was to 
examine the rate of the studies that included elements of professional development in EC and ECSE leadership. Based on the findings, of 124 identified studies, a total of 5 studies (4.1\%) focused on professional development in both fields of EC and ECSE. Of those 5 studies, 4 studies (3.2\%) addressed the field of EC and only 1 study (0.8\%) was conducted in the field of ECSE. In addition, of all 5 studies only 1 study $(0.8 \%)$ implemented a leadership preparation program, and the other 4 studies (3.2\%) used qualitative approach to investigate the professional development needs of staff, the impact of professional development on leaders' performance, and implementation of professional learning and reflective practices. The only descriptive study on professional development in the field of ECSE elaborated on the reciprocal trust in leadership and the responsibility of leaders in building and sustaining organizational culture and climate.

The findings of the systematic review supported the limited, still diverse research literature in leadership in EC and more considerably in ECSE and the need for more need-based and evidence-based leadership research to guide leadership demonstration and development in the field. Below, specific implications drawn from the reviewed literature along with the findings from the systematic review is discussed.

\section{Taking a Stance on National Leadership Trends, Issues, and Practices}

\section{Lack of Targeted Leadership Research}

Based on the reviewed literature in this chapter, the main conclusion is that there is a paucity of high-quality current research on leadership in EC, and more specifically in the field of ECSE. There is little published on effective approaches or models for how to build, support, and sustain leadership capital across all domains of practice and levels of 
EC and ECSE systems. Further, there is an apparent gap of evidence in areas such as leadership evaluation, efficacious models of leadership, and strategies for leadership development.

The field of EC and ECSE is complex because of its diversity, scale, and strong advocacy for family and community roles. This points to the need for more collaborative ways of working and leading which has been addressed by very few studies in the field. In the field of ECSE, having a collaborative and consultative approach in leadership was not only emphasized by the DEC position statement in leadership (DEC, 2015), but also is among the eight topic areas of the DEC-recommended practices (DEC, 2014).

Much of the existing literature on leadership in the fields of EC and ECSE is anecdotal and, in most cases, does not include the strategy-building and professional development models that explore what is meant by effective leadership in EC and ECSE and, by association, how leaders could be equipped to be more effective. Aubrey et al. (2013) and Dunlop (2008) argue the apparent lack of different types of research evidence on EC leadership, which applies to ECSE as well, including case studies, large-scale quantitative studies of leadership implementation, and similarly large-scale studies of specific leadership practices at the program, school, and district level.

Aubrey et al. (2013) and Dunlop (2008) also argued the need for introductory research on the skills and knowledge held by or perceived to be important for good leadership in the field to be used as a baseline for development of actual leadership practices. This is of great importance considering the dearth of literature in the field of ECSE specifically. A quality evidence-based leadership implementation and development in EC and ECSE cannot happen unless there is information regarding the landscape of the 
existing leadership status in the field. Knowledge of the current state can inform databased decision making in terms of future planning for leadership development and sustainability while putting the specific features and needs of the EC and ECSE leadership workforce in priority.

\section{Lack of Data-informed Leadership Development}

The second main conclusion is the clear lack of leadership professional development programs in the field of EC and ECSE. The issue of leadership development programs was mentioned by some seminal studies, yet not many current studies specifically addressed this issue. In a survey study by Bloom (1997), most of the 257 surveyed EC directors said they had received no prior professional development on leadership and management before taking on directorships, and 70\% felt ill-prepared for the challenges that awaited them. Rodd (1997) reported that many EC leaders have received very little management professional development, usually limited to short courses, and in one study, described themselves as feeling uncomfortable with the professional development aspects of their role.

Muijs et al. (2004) argued that most of leadership development programs are small scale and localized, and unlike the schools' sector, there are no national professional development programs in the field of EC. Most leaders also support themselves with necessary professional development needs by taking part in workshops at early childhood conferences or participate in events outside the field of EC (Muijs et al., 2004). The same problem has been highlighted in a study conducted by Movahedazarhouligh and Banerjee (2018) in which the EC and ECSE leaders in one midwestern state in the U.S. claimed self-preparing through conferences and national 
summits rather than receiving a systematic professional development program offered by the state. The recruited leaders in the study also reported being more involved with managing budgets and other issues to meet their programs' logistic needs than with taking part in professional development events.

Even the studies that actually implemented professional development programs and investigated the outcomes are among the seminal ones. In one of those studies conducted by Bloom (1992), 22 leaders who were working in Head Start centers were recruited for a 16-month leadership professional development program using a pre-posttest design in the U.S. The program focused on leadership styles, organizational theory, legal and fiscal issues, relations with parents and the community, child development, selfknowledge, research, and technology. Analyses showed an improvement in organizational climate, improved teaching quality, and an improvement in self-rated knowledge and competence following the professional development program.

In summary, there is a serious lack of leadership preparation and professional development for EC and ECSE leaders. It is likely that many are significantly underprepared for this role. Research suggested that too often positions of leadership in EC and ECSE settings tend to be held by "accidental leaders" with minimal preparation to carry out their responsibilities (Ebbeck \& Waniganayake, 2003; Rodd, 2005).

Bloom and Abel (2015) suggested that professional development makes a difference in the level of leaders' self-efficacy. After completing preparation programs, leaders with greater levels of leadership preparation report significant gains in their level of competence, and staff who work at these programs perceive the work environment to be more positive and productive (Bloom, 2014; Bloom \& Bella, 2005). Leadership 
preparation coupled with a strong mentoring component results in demonstrable organizational change (Doherty, 2011). Bella (2013) found that leaders with more preparation report significant differences in both their self-perceptions as leaders and in their leadership practices for the EC and ECSE workforce.

The findings of this literature review also shed light on the fact that much of the existing research has investigated leadership within the EC domain, and a very small fraction addressed the field of ECSE. There is a well-articulated position statement on leadership in ECSE (DEC, 2015) and a set of recommended practices (DEC, 2014) to provide guidance to practitioners about effective ways to promote the development of young children with or at risk for disabilities. Both the DEC position statement on leadership and the DEC-recommended practices in leadership can be used as guiding tools to build, maintain, and sustain leadership capital in ECSE through collaborative, team-based efforts.

\section{Next Steps}

Leadership continues to be regarded as an important professional issue for the fields of EC and ECSE. In the quest for increasing quality in EC classrooms, many EC and ECSE professionals consider leadership to be the key element. Yet, despite continued interest and concern about leadership in EC and ECSE, key questions continue to be asked by members of the fields about the nature of leadership. This literature review revealed that the fields of EC and more specifically ECSE need to develop a more extensive knowledge base and theoretical framework concerning roles and competencies specific to leadership as well as strategies for developing the leadership skills needed for these roles. Leadership in EC and ECSE is a complex concept, and its role in quality 
service provision to young children and their families has not been well understood and practiced to date. If we look beyond EC and ECSE literature, much has been written about school leadership and wider educational leadership. Given the centrality of EC in government policy and its current high profile, it seems almost inconceivable that the leadership practices of those working within EC systems are not being taken seriously (Aubrey et al., 2013).

Taking what research indicates about the relationship between leadership and positive organizational outcomes, this would seem to be a most serious oversight for two reasons: (a) first, because it leaves effective leadership practices to chance and implies that there will inevitably be inefficient leaders; and (b) second, because it knowingly leaves those in leadership positions unprepared for the significant leadership tasks they face on a daily basis. These seem to be two compelling reasons for investing substantially in leadership research and development for EC and ECSE. All of the evidence suggests that this investment is long overdue.

Research findings that are organized, accessible, and easily applied by leaders and practitioners can enhance the likelihood of effective EC and ECSE leadership development. However, despite the scant leadership research in EC and more specifically in ECSE, the future of the fields over the next decade appears promising. Today, we have more resources, policies, and professional support for quality EC and ECSE programs than ever before. Recent reforms call for stronger constructions of leadership across diverse EC and ECSE settings. Leadership that is both pedagogically focused on young children and sociologically focused on families and the communities must interconnect with integrated services for children, families, and communities. As the DEC position 
statement in leadership (2015) emphasized, it's time for the field to deliberately engage in identifying specific competencies necessary to be an effective leader as well as the organizational cultures necessary to promote and sustain high-quality leadership in EC and ECSE. Also as mentioned in the first chapter, according to the DEC position statement in leadership (2015), if the field aims to develop effective and efficient leadership, it needs to be based on "current construct and demonstration of leadership skills across service systems" (p. 1) by which leadership development in the filed can be informed and knowledge base on effective leadership practices can be improved.

\section{Purpose of the Study}

This dissertation study aimed to take a step forward to address one of the many identified gaps discussed in this chapter, which is specifically emphasized by the DEC position statement on leadership (2015). The purpose of this study was to improve the understanding of the current construct and demonstration of leadership in EC and ECSE by district-level and county-level EC and ECSE leaders in the state of Colorado to identify: (a) who the leaders are in terms of their leadership qualifications and background; (b) the state of current leadership implementation; (c) the challenges and barriers that affect the EC and ECSE leaders' performance; and (d) the support these leaders receive and further need to practice quality leadership development and sustainability in the field. Such understanding would guide effective and responsive planning in local, state, or federal levels. Such an investigation may also help unravel the areas in need of improvement in higher education preparation programs through targeted program development to meet the needs of the EC and ECSE workforce. 


\section{Summary of the Chapter}

This chapter summarized the literature related to leadership theories and leadership models in general and in an EC and ECSE context. Classical leadership theories were discussed, and different leadership models that have been studied in EC and ECSE context were presented. The Whole Leadership Framework and its sub-domains were also explored.

The chapter also examined leadership research trends in EC and ECSE. Research trends indicated the need for more research that examines leadership capital, leadership evaluation, and data-informed leadership development and preparation in the field. The 2018 L.E.A.D ECC report provided some information regarding the existing academic leadership programs in the nation. The data from this report also highlighted the need for a united definition of the leadership competencies and qualification for the leadership workforce in the field.

Based on the investigated gap in the literature and the calls for more research, the chapter emphasized the areas that require further investigation, including the specific objectives of this study to identify current construct and demonstration of leadership in the field to inform data-driven leadership development and sustainability. The information gained in this review of the literature provides the foundation for the design and methods used in this study that are described in the following chapter. 


\section{CHAPTER III}

\section{METHODOLOGY}

\section{Introduction}

This chapter describes the research design that was used to carry out this dissertation study. The chapter begins with a description of the early childhood (EC) and early childhood special education (ECSE) services and programs in the state of Colorado. The chapter continues with a presentation of the purpose of the study, the researcher's stance, and the research questions followed by a description of the research design, sampling, instrumentation, and the data collection procedures. The final section explains the process for analysis of data that was used.

\section{Early Childhood and Early Childhood Special Education in Colorado}

There are different EC programs in the state of Colorado including childcare programs, early intervention (EI) programs, family support programs, Colorado Peak, Head Start and Early Head Start (HS/EHS) programs, and the Colorado Preschool Program. This study investigated EC and ECSE leaders from state and federally funded programs. The programs include: (a) Early Intervention Colorado that provide services under Part C of the Individuals with Disabilities Education Act (IDEA) (2004), (b) Colorado Preschool Special Education that provide services under Part B, Section 619 of 
IDEA (2004), (c) Colorado Preschool Program (CPP) that is a state-funded program, and (d) Colorado HS/EHS Programs that is a federally funded program.

It should be noted that through communication with preschool special education state level leaders at the Colorado Department of Education, it was clarified that preschool special education services are coordinated by the CPP directors and/or Child Find coordinators, and there is not a specific position as IDEA, Part B, Section 619 director/coordinator to provide services to children with special needs.

\section{Early Intervention Colorado}

Early Intervention Colorado, under the Office of Early Childhood, Colorado Department of Human Services, is Colorado's Lead Part C agency that is responsible for providing early intervention services under the IDEA (2004). Colorado's EI program provides supports and services to children with developmental delays or disabilities and their families from birth until the child's third birthday (Colorado Office of Early Childhood, 2019). Early Intervention Colorado can help families learn ways to support and promote their child's development within their everyday routines and activities. By providing needed services and supports during this time, families may be able to help their children with special needs develop to their full potential and may decrease the need for additional help later in life. The program can also help families learn ways to support and promote their child's development within their family's daily activities and community life (Colorado Office of Early Childhood, 2019).

Community centered boards (CCBs) are local agencies throughout the state of Colorado that are responsible for providing EI services to children with developmental delays or disabilities and their families from birth until the child's third birthday in their 
local service area. According to Early Childhood Training and Technical Assistance System (2019), 7,635 children ages birth through 3 were served under the IDEA Part C services in the state of Colorado during 2018.

\section{Preschool Special Education Services in Colorado}

Currently about 14,293 children receive individualized special education services in preschool in the state of Colorado (Early Childhood Training and Technical Assistance System, 2019). Services are provided through school-based programs, community early childhood settings, and/or Head Start Programs (Colorado Department of Education, 2019). According to the $40^{\text {th }}$ annual report to congress on the implementation of the IDEA (2018) citation, 5.4\% of the population ages 3 through 5 served under the IDEA, Part B, Section 619, with 15.2\% increase in percent change between 2008 and 2016 in state of Colorado.

Child Find is part of Colorado's Department of Education system and preschool services for identifying children suspected of having a delay in development. The purpose of the evaluation is to determine if there is a significant delay and/or if there is a need for early intervention or special education services. For children birth to 3 years of age, Child Find teams partner with local EI Colorado programs that provide service coordination and eligibility determination. Children 3 to 5 years old are evaluated as a part of the preschool special education process.

\section{Colorado Preschool Program}

The Colorado Preschool Program (CPP), under the Colorado Department of Education, was launched in 1988 in an effort to target 3- and 4-year-olds in need of language development support, with the goal of lowering school dropout rates and 
reducing dependence on public assistance. In 1992, CPP became a permanent program and expanded its target population to all children who lack overall school readiness due to family risk factors. Risk factors for eligibility are defined in statute and include: eligibility for free or reduced-price meals; being a dual language learner; being in foster care; having a family history of abuse or neglect, homelessness, parental substance abuse; or having a teen parent as well as locally determined risk factors. Four-year-olds must meet at least one risk factor to enroll in CPP, while 3-year-olds are required to meet a minimum of three risk factors (Colorado Department of Education, 2019).

In the 2016-2017 school year, $98 \%$ of the 179 school districts in Colorado offered the CPP. Two types of slots can be allocated to districts: CPP slots, which can be used to serve eligible pre-K students, and Early Childhood At-Risk Enhancement (ECARE) slots, which can be used to serve pre-K students or to provide full-day opportunities for eligible kindergartners (Colorado Department of Education, 2019).

Schools may subcontract with private childcare centers, Head Start, or other community-based or public agencies. The Charter School Institute also participates in the $\mathrm{CPP}$ as a direct recipient of funds. Funding is awarded competitively with priority given to districts not currently participating in CPP. The CPP may use additional funding sources, such as federal Head Start money, to extend the program day, supplement services, or provide wraparound care (Colorado Department of Education, 2019).

The CPP funding is determined through the Colorado school finance formula, with preschoolers receiving half the number of students in Grades 1 through 12. These funds are distributed directly to public schools, which are given a predetermined number of half-day slots to serve eligible children (Colorado Department of Education, 2019). 


\section{Colorado Head Start and Early Head Start Program}

Head Start is a federal program that promotes the school readiness of children from birth to age 5 from low-income families by enhancing their cognitive, social, and emotional development. Head Start programs provide a learning environment that supports children's growth in many areas such as language, literacy, and social and emotional development. Head Start emphasizes the role of parents as their child's first and most important teacher. These programs help build relationships with families that support family well-being and many other important areas such as children's growth and development in a positive learning environment through early learning and health services (U.S. Department of Health and Human Services, 2019).

Many HS programs also provide EHS which serves infants, toddlers, and pregnant women and their families who have incomes below the federal poverty level. Children from birth to age 5 from families with low income, according to the Poverty Guidelines published by the federal government, are eligible for HS and EHS services. In order to qualify, families must have an annual household income (before taxes) that is below the certain amounts, established by the federal agencies (U.S. Department of Health and Human Services, 2019). Children in foster care, homeless children, and children from families receiving public assistance (Temporary Assistance for Needy Families or Supplemental Security Income) are also eligible for HS and EHS services, regardless of income (U.S. Department of Health and Human Services, 2019).

Head Start and EHS programs offer a variety of service models, depending on the needs of the local community. Many Head Start and EHS programs are based in centers and schools. Other programs are located in childcare centers and family childcare homes. 
Some programs offer home-based services that assign dedicated staff who conduct weekly visits to children in their own home and work with parents (U.S. Department of Health and Human Services, 2019). According to the Early Childhood Training and Technical Assistance System (2019), 8,721 children participated in HS/EHS programs during 2018 in the state of Colorado.

\section{Purpose of the Study and Research Questions}

This study aimed to investigate leadership demonstration by district-level and county-level EC and ECSE leaders in the state of Colorado to identify: (a) who the leaders are in terms of their leadership qualifications and background; (b) the current state of leadership implementation; (c) the challenges and barriers that affect the EC and ECSE leaders' performance; and (d) the support these leaders receive and further need to practice quality leadership development and sustainability in the field. The following research questions guided this study:

Q1 Who leads district-level and county-level early childhood and early childhood special education programs? What leadership competencies and academic and professional background do they have?

Q2 How do district-level and county-level early childhood and early childhood special education leaders evaluate their leadership implementation?

Q3 What challenges do these leaders encounter in leadership implementation and development in their programs?

Q4 What professional development and support do these leaders need to implement quality leadership in their practice?

Q5 How do state-level leaders perceive the leadership implementation, challenges, and professional development needs of district-level and countylevel early childhood and early childhood special education leaders? 


\section{Researcher Stance}

My personal interest in this topic originated from my background as a rehabilitation director following my Master of Science in Rehabilitation Administration in my home country Iran that provided me with the experience of directorship in service provision for individuals with disabilities. Those experiences helped me delve deeper into the concept of leadership and the impact of quality leadership on the positive outcomes for children and individuals with disabilities and their families. One of my primary studies in my doctoral program addressed quality leadership in family-centered service delivery, which was a good start to pursue my passion in the topic of leadership. I also attended professional events in the field such as the Division for Early Childhood annual conferences and had the opportunity to connect and network with leaders in the field. So far, I have had the opportunities to grow as an emerging leader and develop my keen interest to target this topic for my dissertation study.

It is said that "It takes a village to raise a child," but what does it take to create a village? I sincerely believe that quality leadership through evidence-based knowledge, collaboration, cooperation, and communication can help build that village and strengthen the whole system. Particularly in the fields of EC and ECSE with a series of diverse environments, programs and institutions, we have yet to develop an inclusive concept of leadership that takes the needs at all levels and in all areas into account. If we are ambivalent about the need for a unified conceptualization of leadership within the fields of EC and ECSE, those on the outside looking in are even more confused. This makes developing future leaders in such an environment more difficult. If we need leaders in our profession, we must be willing to help develop and reward them. We must acknowledge 
the existing leaders in our profession and take steps to develop stronger ones for the future.

\section{Research Design}

A mixed-methods research design, utilizing the strengths of both quantitative and qualitative research methods, was used in this study (Creswell, 2014). A mixed method approach allows for collection of both qualitative and quantitative data, which provides researchers with the ability to increase the quality and quantity of interpretations of the data (Onwuegbuzie \& Leech, 2006).

\section{Assumption of and Rationale for the Selected Research Design}

A mixed-methods study provides the opportunity for more insight through the combination of characteristics of quantitative and qualitative research as opposed to the use of either method alone (Creswell \& Tashakkori, 2007). In addition, the type of information gained from using each method varies. For example, quantitative data allow for statistical comparison of individuals and groups and often provide participants with a variety of responses to choose from when answering specific questions. Considerations of quantitative methodology include that it is deductive, the researcher is removed from the data completion, and it is more generalizable and population oriented (Creswell \& Tashakkori, 2007; Day, Sammons, \& Gu, 2008; Johnson \& Onwuegbuzie, 2004).

Qualitative data often provide more depth and understanding with responses (Creswell, 2014). Considerations of qualitative methodology include that it is inductive, the researcher is close to the data, it tends to be less generalizable because of the smaller sample size, it provides more depth and layers of responses, and it lacks statistical 
conclusions (Creswell \& Tashakkori, 2007; Day et al., 2008; Johnson \& Onwuegbuzie, 2004).

According to Creswell (2014), the explanatory sequential mixed methods design is one in which the researcher first conducts quantitative research, analyzes the results, and then builds on the results to explain them in more detail with qualitative research. It is considered explanatory because the initial quantitative data results are explained further with the qualitative data. It is considered sequential because the initial quantitative phase is followed by the qualitative phase (Creswell, 2014). Please see Figure 1 for an overview of the study framework. 
Purose: To investigate district level and county level EC and ECSE leaders' competencies and their needs and challenges in state of Colorado to improve the understanding of current construct and demonstration of leadership and necessary required supports for leadership develop.
Participant recruitment for Phase I

Phase I: Disseminate online survey to district level and county level EC and ECSE leaders in the state of Colorado

a. Community Certified Board (CCB) leaders at district and county levels

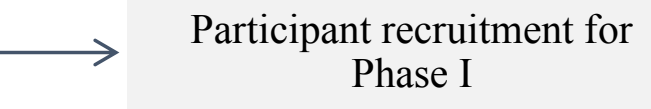

b. EC/ECSE leaders at districts

c. HS/EHS leaders in counties

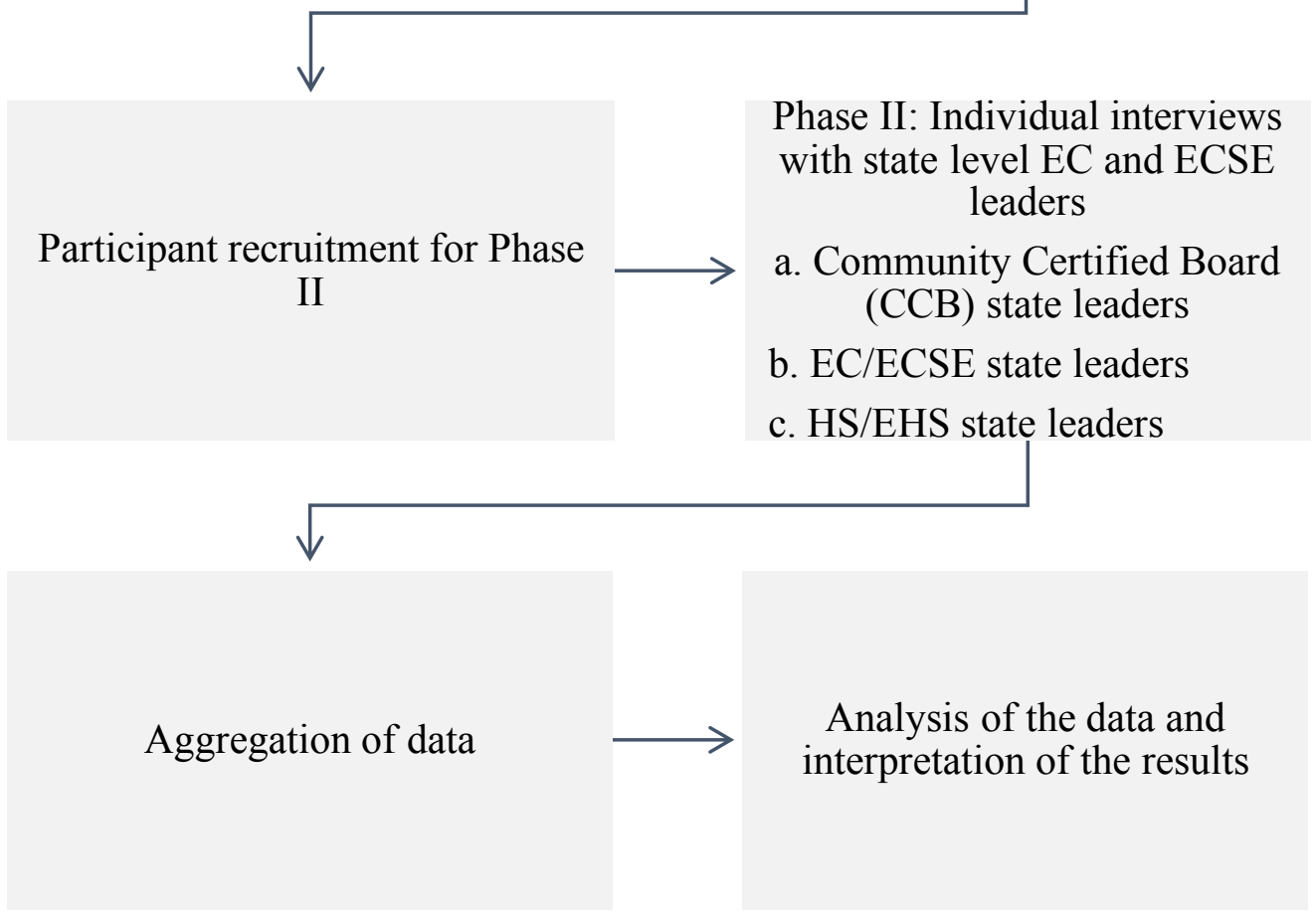

Figure 1. Overview of study framework. 


\section{Participants and Sampling}

The study sampling pool in both Phase I and Phase II included 294 EC and ECSE leaders from state and federally funded EC and ECSE programs in THE state of Colorado. The sampling pool for Phase I included 290 district-level and county-level EC and ECSE leaders and 4 state-level EC and ECSE leaders for Phase II. Below, target populations specific to each phase is described.

\section{Phase I: Quantitative--Online} Survey

Target population. Two hundred ninety $(n=290)$ district-level and county-level EC and ECSE leaders were requested to participate in the survey (Phase I). The districtlevel and county-level EC and ECSE leaders included individuals in three different practices as listed below. It should be mentioned that $60(n=60)$ overlaps were identified across the directors from different agencies, and the final overlap free number of EC and ECSE district-level and county-level directors was identified as 290.

1) Community Center Board (CCB) directors $(n=20)$ from EI Colorado.

2) Program directors $(n=275)$ from Colorado Preschool Special Education and CPP. As mentioned earlier, preschool special education services in the state of Colorado are directed either by CCP program directors and/or Child Find coordinators.

a) Colorado Preschool Program and preschool special education directors $(\mathrm{N}$ $=175)$

b) Child Find coordinators $(n=100)$

3) Program directors $(n=55)$ from HS/EHS. The $55 \mathrm{HS} / \mathrm{EHS}$ program directors include 30 directors responsible for only HS services, 10 directors responsible 
for only EHS services, and 15 directors responsible for both HS/EHS services. Involvement in either of these state and federally funded programs as a program director defined the inclusion criteria for the participants in Phase I.

It should be mentioned that the total number of directors as stated above comes to 350. However, 60 overlaps were identified across the directors from different agencies and the final overlap free number of EC ad ECSE district level and county level directors was identified as 290. Involvement in either of these state and federally funded programs as a program director, defined the inclusion criteria for the participants in Phase I.

Sampling method. Convenience sampling was used to recruit participants for Phase I. Convenience sampling is used when members of the target population that meet certain practical criteria, such as easy accessibility, geographical proximity, availability at a given time, or the willingness to participate are included for the purpose of the study (Teddlie \& Yu, 2007). A sampling frame (contact list) for CPP directors and Child Find coordinators $(n=276)$ is readily available on the CDE website. For CCB directors $(n=$ 20) such a sampling frame is available on the Colorado Office of Early Childhood website. For HS/EHS directors $(n=55)$, the Head Start Collaboration Office was contacted and a sampling frame for HS/EHS directors in the state of Colorado was obtained.

\section{Phase II: Qualitative--Individual Interviews}

Target population. Four $(n=4)$ state-level EC and ECSE leaders from the aforementioned state and federally funded programs (CCB, CPP/Preschool ECSE, HS/EHS), 1 from each program, participated in individual qualitative interviews. 
Involvement in any of these state and federally funded programs as a state-level leader defined the inclusion criteria for the participants in Phase II.

Sampling method. Convenience sampling was also used to recruit participants for Phase II individual interviews. Using the EC and ECSE state leaders' contact information on the CDE websites, Colorado Office of Early Childhood, and Head Start Collaboration Office, the leaders were contacted and invited to participate in the individual interviews.

\section{Data Collection Procedures}

Two data collection instruments, including an online survey and individual interviews, were used for this study to provide an in-depth understanding of the EC and ECSE leaders' qualifications in their leadership position, the current state of leadership implementation, the challenges they face in their leadership practices, and the leaders' perceived needs for leadership development. The online survey was distributed among district- and county-level EC and ECSE leaders, and the individual interviews were conducted with state-level EC and ECSE leaders.

\section{Phase I: Instrumentation}

A cross-sectional online survey was used to explore the research questions. The survey responses were collected using Qualtrics (C Qualtrics Labs, Inc., 2019). The survey was divided into three sections.

Survey Section 1. The first section included 18 questions about the leaders' academic and professional background and their leadership qualifications to meet their job requirements. The first section aimed to answer the first research question in terms of understanding who the leaders in charge of district- and county-level EC and ECSE 
programs are, what type of academic and professional background they have, and if their leadership qualifications meets their job requirements. The 18 questions in Section 1 included items addressing the district-level and county-level leaders' demographic characteristics (e.g., gender, years of experience, age group, etc.) and academic and professional background characteristics (e.g., the leaders' roles and responsibilities, professional agency, leadership qualifications, etc.).

Survey Section 2. The second section of the survey aimed to assess: (a) the current state of leadership implementation by district-level and county-level EC and ECSE leaders; (b) the challenges that these leaders encounter in implementing leadership practices; and (c) the professional development needs the leaders think they need in each leadership practice. These three domains are the core ideas in Research Questions 2, 3, and 4 in this study.

Section 2 of the survey included 26 recommended leadership practices, drawn from Leadership Performance Checklists (Early Childhood Technical Assistance Center [ECTA], 2019). These recommended leadership practices were developed based on the Division for Early Childhood (DEC) Recommended Practices in Leadership by the ECTA. The DEC Recommended Practices were developed to help translate research into practice and to ensure that children with or at risk for developmental delays or disabilities, their families, and the personnel who support them have access to practices that result in better outcomes (Barton \& Smith, 2015). Leadership is one of the eight topic areas that provides guidance and includes 14 recommended practices that directly relate to the role of early childhood leaders and administrators. See Table 1 for on outline of the 14 DEC Recommended Practices in leadership. 
Table 1

DEC Recommended Practices in Leadership

No. Leadership Practices

L1 Leaders create a culture and a climate in which practitioners feel a sense of belonging and want to support the organization's mission and goals.

L2 Leaders promote adherence to and model the DEC Code of Ethics, DEC Position Statements and Papers, and the DEC Recommended Practices.

L3 Leaders develop and implement policies, structures, and practices that promote shared decision making with practitioners and families.

L4 Leaders belong to professional association(s) and engage in ongoing evidence-based professional development.

L5 Leaders advocate for policies and resources that promote the implementation of the DEC Position Statements and Papers and the DEC Recommended Practices.

L6 Leaders establish partnerships across levels (state to local) and with their counterparts in other systems and agencies to create coordinated and inclusive systems of services and supports.

L7 Leaders develop, refine, and implement policies and procedures that create the conditions for practitioners to implement the DEC Recommended Practices.

L8 Leaders work across levels and sectors to secure fiscal and human resources and maximize the use of these resources to successfully implement the DEC Recommended Practices.

L9 Leaders develop and implement an evidence-based professional development system or approach that provides practitioners a variety of supports to ensure they have the knowledge and skills needed to implement the DEC Recommended Practices.

L10 Leaders ensure practitioners know and follow professional standards and all applicable laws and regulations governing service provision.

L11 Leaders collaborate with higher education, state licensing and certification agencies, practitioners, professional associations, and other stakeholders to develop or revise state competencies that align with DEC, Council for Exceptional Children (CEC), and other national professional standards.

L12 Leaders collaborate with stakeholders to collect and use data for program management and continuous program improvement and to examine the effectiveness of services and supports in improving child and family outcomes.

L13 Leaders promote efficient and coordinated service delivery for children and families by creating the conditions for practitioners from multiple disciplines and the family to work together as a team.

L14 Leaders collaborate with other agencies and programs to develop and implement ongoing community-wide screening procedures to identify and refer children who may need additional evaluation and services. 
The ECTA Center is a national technical assistance center focused on building state and local system capacity to improve outcomes for children with disabilities and their families. The ECTA Center is funded by a cooperative agreement with the Department of Education's Office of Special Education Programs (OSEP).

The 26 recommended leadership practices include three sub-sections: (a) vision and direction in leadership, (b) motivation and guidance in leadership, and (c) collaboration in leadership. Each section includes examples of steps leaders can take to create a well-functioning and forward-thinking organization and to help practitioners feel a sense of belonging as they understand their purpose within the organization. According to the ECTA Center (ECTA, 2019), the checklist can be used as a self-evaluation by leaders at both state and local levels.

Each of the 26 leadership practices in Section 2 of the survey were used to assess the three aforementioned domains (leadership implementation, challenges, and professional development needs) at the same time. Participants rated (a) the extent to which they use each of the recommended leadership practices, (b) how challenging it is to apply and implement each of the practices, and (c) what level of professional development need they have in each practice.

For each domain, participants rated each practice using three side by side, 4-point Likert scales as depicted below:

- The current state of leadership implementation: $1=$ seldom or never $(0-25 \%)$, $2=$ some of the time $(25-50 \%), 3=$ as often as I can $(50-75 \%)$, and $4=$ most of the time $(75-100 \%)$. 
- Leadership implementation challenges: $1=$ not challenging, $2=$ somewhat challenging, $3=$ challenging, and $4=$ very challenging.

- Leadership professional development needs: $1=$ low professional development need, 2 = medium professional development need, $3=$ high professional development need, and $4=$ very high professional development need.

The three scales (implementation, challenges, and professional development need) were presented side by side in the actual Qualtrics survey. However, in Appendix A that outlines the online survey, due to lack of space, the three scales are presented in rows.

Survey Section 3. The third and final section of the survey included five openended questions that asked participants to provide (a) how they evaluate leadership implementation in their programs, (b) any comments they have regarding leadership competencies/practices, (c) any other perceived challenges they regularly encounter in their role that have not been addressed in the survey, (d) any other perceived professional development needs the leaders have in their role that have not been addressed in the survey, and (e) any other perceived factors the leaders believe make a considerable contribution to leadership development and sustainability in the field. See Appendix A for a copy of the proposed survey.

Validity and reliability. Validity is the degree to which an instrument produces scores that measure what the instrument is intended to measure (Creswell, 2014). Content clarity and appropriateness were assessed by having the survey reviewed by five experts who are knowledgeable about the field of EC and ECSE, the concept of leadership in EC, and ECSE and survey creation. They included a university professor and expert in special 
education, three state-level experts in the field of EC and ECSE, and a university expert in research and survey design. Based on the recommendations from the panel of experts, the modifications were applied accordingly. The recommendations included rewording and merging some of the demographic items to better represent the EC/ECSE workforce in the state of Colorado.

Reliability refers to the consistency of scores with which an instrument obtains the desired information (Creswell, 2014). Prior to actual data collection, the survey was piloted by 8 representatives of each group (EI, EC, and ECSE) from five different states (Texas, Nevada, Florida, New York, Utah), and modifications were applied based on the results and recommendations. The modifications included rewording the questions to better convey the purpose of the three scales of implementation, challenges and PD needs.

\section{Phase I: Quantitative Data Collection}

After securing approval to complete this research study by the Institutional Review Board of the University of Northern Colorado (Appendix E), the following steps were completed to collect data. First, a week prior to actual dissemination of the survey, a notification email was sent to the participants, notifying them that they would be receiving a survey with some specific research purposes, encouraging them to consider completing the survey. Second, a week after the notification email, an email of introduction and the anonymous link for the online survey instrument was sent to each potential participant using Qualtrics (C Qualtrics Labs, Inc., 2019). Both the notification email and the cover letter explained the purpose of the research study and advised individuals of the voluntary nature of participation, affirming that there would be no 
negative consequences for the participant if he/she chose not to participate in the study. The anonymous link to the online survey was included in the introductory email. Permission to take part in the study was obtained through a consent form which was the initial page of the online survey (Appendix B). Respondents implied their consent to participate in the study by choosing to continue to complete the survey. Third, a weekly follow-up email was sent to the potential participants, thanking them and asking them to complete the survey if they had not yet completed it. Also, efforts were made to encourage state-level leaders (CPP, EI, HS/EHS) to send a separate reminder to the contact list, encouraging the district-level and county-level EC and ECSE leaders to participate in the study. The survey was closed at the end of the fifth week.

\section{Phase II: Instrumentation}

Individual qualitative interviews were used to explore state-level leaders' perspectives in terms of the research questions proposed by this study. The interview protocol was developed based on the primary results from Phase I. The interview protocol included more in-depth open-ended questions to gain an understanding of the state-level leaders' perspectives regarding leadership qualifications, leadership implementation, and challenges and professional development needs of district-level and county-level EC and ECSE leaders. Please see Appendix C for an initial outline of proposed interview questions.

\section{Phase II: Qualitative Data Collection}

Interview participants were four state-level leaders from each of the aforementioned EC and ECSE programs (EI Colorado, CPP and Child Find, and HS/EHS). The participants who gave their informed consent to participate in the study 
received a cover letter of introduction explaining the purpose of the study, the anticipated obligation of time necessary to conduct the interview, the assurance of anonymity for all participants, and a reminder that participants were free to discontinue the study at any time. The researcher conducted remote telephone interviews with the participants using the interview protocol designed for this study. The interviews were each completed in 45 minutes to one hour. Interviewees signed a consent form (Appendix D) that was sent to them via email. Interviews were digitally recorded with the consent of participants. The digital recordings from the individual interview conversations were transcribed verbatim by a professional company.

\section{Data Analysis Procedure}

\section{Phase I: Quantitative Data Analysis}

The quantitative data included the results of the survey that was distributed and collected using Qualtrics (C Qualtrics Labs, Inc., 2019). The data from the online survey were uploaded to the Statistical Package for the Social Science (SPSS) (C IBM Corporation, 2019) Version 24 to determine descriptive statistics including frequencies and mean score percentages for the categorical variables and the Likert items.

In addition to descriptive data analysis, inferential statistical analysis was also conducted to reach conclusions that extend beyond the immediate data alone and make inferences from the data to more general conditions. The inferential analysis included: (a) bivariate correlations and exploratory factor analysis (EFA) to gain a better understanding of the underlying constructs and patterns among the leadership practices, (b) Cronbach's Alpha to inform the internal consistency and reliability of scores, and (c) multivariate analysis of variance (MANOVA) to determine whether there were any 
statistically significant differences between the means of the groups in the survey and also to see if certain demographic characteristics explained the differences in identification of leadership implementation, challenges and professional development needs by the leaders. Based on the potential method of statistical analysis of the data and using the G*power software (Faul, Erdfelder, Buchner, \& Lang, 2009) for calculating estimated sample size to control for statistical power of the study, a priori statistical power for $\mathrm{F}$ test, with a medium effect size (0.3), power of 0.8 , alpha level of .05 , and number of groups as 5, a sample size of 148 was considered adequate. Regarding the open-ended questions in the survey, ideas and concepts across and within transcripts were extracted. The repeated themes were tagged with codes and a code table of descriptive keywords and phrases was created for each theme (Hatch, 2002).

\section{Phase II: Qualitative Data Analysis}

Qualitative studies attempt to gain understanding by uncovering meanings from participants' responses (Creswell, 2007). Interview transcripts were analyzed using the Qualitative Content Analysis protocol described by Graneheim and Lundman (2004). Qualitative Content Analysis was deemed an appropriate technique for analyzing the interview data, as it stays close to participants' words while simultaneously summarizing the data and underlying meanings. The responses were categorized and coded using NVivo11. In the first stage of analysis, text relevant to the research aims was identified and labeled as meaning units. These meaning units were phrases, sentences, or paragraphs spoken by the state leaders, and excluded conversational fillers and unrelated humor. In the second stage of analysis, these meaning units were then condensed using words that closely approximated the participants' words. Efforts were made for the 
condensed meaning units as being accurate representations of the participant's words. In the third stage of analysis, the condensed meaning units were interpreted to form codes. The fourth stage of analysis involved grouping codes into mutually exclusive categories based on shared meaning. Finally, themes were developed that connected the underlying meaning of categories.

Procedures recommended by Brantlinger, Jimenez, Klingner, Pugach, and Richardson (2005) were used to ensure the trustworthiness and credibility of the qualitative data. Lincoln and Guba (1985) also proposed certain operational techniques to improve the credibility, transferability, dependability, and confirmability of qualitative studies, and among those are investigator triangulation of the data and transcript review as an aspect of member checking. Member checking covers a range of activities including returning the interview transcript to participants, a member check interview using the interview transcript data or interpreted data, a member check focus group, or returning analyzed synthesized data (Birt, Scott, Cavers, Campbell, \& Walter, 2016; Harper \& Cole, 2012).

Within an objectivist epistemology, asking a participant to check the transcript of their interview potentially enhances accuracy of the data. Yet within a constructionist epistemology, it can be used as a way of enabling participants to reconstruct their narrative through deleting extracts they feel no longer represent their experience or that they feel presents them in a negative way. Providing opportunities to delete data calls into question the very nature of research data: Are research data "owned" by the researcher or does it always "belong" to the participant? This method of member checking enables the 
researcher to make claims about the accuracy of the transcription of the interview (Birt et al., 2016; Harper \& Cole, 2012).

The researcher asked the participants to review the transcripts and add comments or clarifications to ensure that the transcript accurately reflected their opinions. Investigator triangulation was also conducted to develop a broader and deeper understanding of how the different investigators view the phenomenon (Creswell, 2007). Investigator triangulation involves using several different investigators in the analysis process wherein each investigator examines the program with the same qualitative method (interview, observation, case study, or focus groups). The findings from each evaluator are then compared. If the findings from the different evaluators arrived at the same conclusion, then confidence in the findings would be heightened (Creswell, 2007; Merriam, 2009). To conduct investigator triangulation, the researcher randomly selected two audio interview transcripts and asked a doctoral level colleague to listen to the interviews and do the coding procedure for $30 \%$ of each interview to ensure that the protocol for each interview was followed consistently. No major disagreement occurred between the

\section{Summary of the Chapter}

This proposed study used an explanatory sequential mixed methods design through a quantitative survey (Phase I) with EC and ECSE district and county level leaders and qualitative individual interviews (Phase II) with EC and ECSE state level leaders. Quantitative data analysis included descriptive and inferential statistical analyses. The qualitative data analysis included development of coding tables for the open-ended question in the section three of the survey and Qualitative Content Analysis for individual 
interviews. The quantitative and qualitative data were aggregated and triangulated to reflect the perspective and experiences of each group. 


\section{CHAPTER IV}

\section{FINDINGS}

This chapter presents the findings which are organized by the two quantitative and qualitative phases and the research questions in each phase. The chapter begins with a brief overview of the purpose of the study and continues with presentation of the findings.

\section{Purpose of the Study}

The purpose of this explanatory sequential mixed-methods research study was to investigate leadership practices by district-level and county-level early childhood (EC) and early childhood special education (ECSE) leaders in the state of Colorado to identify: (a) who the leaders are in terms of their leadership qualifications and background, (b) the current state of leadership implementation, (c) the challenges and barriers that affect the EC and ECSE leaders' performance, (d) the support these leaders need for quality leadership development and sustainability in the field, and (e) the state leaders' perceptions regarding leadership implementation, challenges, and professional development needs of the district-level and county-level EC and ECSE leaders. The following research questions guided this study:

Q1 Who leads district-level and county-level early childhood and early childhood special education programs? What leadership competencies and academic and professional background do they have? 
Q2 How do district-level and county-level early childhood and early childhood special education leaders evaluate their leadership implementation?

Q3 What challenges do these leaders encounter in leadership implementation and development in their programs?

Q4 What professional development and support do these leaders need to implement quality leadership in their practice?

Q5 How do state-level leaders perceive the leadership implementation, challenges, and professional development needs of district-level and countylevel early childhood and early childhood special education leaders?

A cross-sectional online survey was used in Phase I with district-level and countylevel EC and ECSE leaders to explore the first four research questions followed by individual interviews in Phase II with state-level leaders to answer the fifth research question. Below findings are presented by each phase of the study.

\section{Phase I: Quantitative Data Analysis--Descriptive Results}

Two hundred ninety $(\mathrm{n}=290)$ district-level/county-level EC and ECSE leaders from state and federally funded programs in the state of Colorado including community center board (CCB) directors, program directors from the Colorado Preschool Special Education and Colorado Preschool Program (Child Find and CPP) and program directors from Head Start/Early Head Start (HS/EHS) were requested to participate in the online survey. One hundred forty-three $(\mathrm{n}=143)$ district-level and county-level EC and ECSE directors participated in Phase I by completing and returning the survey which generated a response rate of $49.3 \%$.

The online survey was divided into three sections: (a) Section1 included 18 demographic questions to answer Research Question 1 in terms of the district-level and county-level EC and ECSE leaders' academic and professional background and their leadership qualifications to meet their job requirements; (b) Section 2 included 26 
recommended leadership practices, drawn from the Leadership Performance Checklists based on the Division for Early Childhood (DEC) of the Council for Exceptional Children (CEC) Recommended Practices (ECTA, 2019) to answer Research Questions 2, 3 , and 4 in terms of leadership implementation, implementation challenges, and professional development needs; and (c) Section 3 included five open-ended questions that asked participants to provide their further comments. The findings in terms of each research question are presented here.

\section{Results: Phase I}

\section{Research Question One}

Q1 Who leads district-level and county-level early childhood and early childhood special education programs? What leadership competencies and academic and professional background do they have?

The first section of the online survey aimed to answer the first research question in terms of understanding who the leaders in charge of district-level and county-level EC and ECSE programs are. The questions in this section asked the leaders about a number of professional characteristics, their academic and professional background, if their leadership qualifications meet their job requirements, and their participation in and provision of professional development activities. Table 2 provides a summary of the district-level and county-level EC and ECSE leaders' demographic, academic and professional characteristics. 
Table 2

Participants' Demographic Characteristics by Gender, Age, Education, and Ethnicity

\begin{tabular}{|c|c|c|c|}
\hline Category & Sub-category & $\begin{array}{c}\text { Frequency } \\
(\mathrm{N})\end{array}$ & $\begin{array}{c}\text { Percent } \\
(\%)\end{array}$ \\
\hline \multirow[t]{4}{*}{ Gender } & Female & 137 & 95.80 \\
\hline & Male & 6 & 3.92 \\
\hline & Other & 0 & 0.00 \\
\hline & I'd rather not say & 0 & 0.00 \\
\hline \multirow{5}{*}{ Age group } & $20-29$ & 2 & 1.40 \\
\hline & $30-39$ & 27 & 18.88 \\
\hline & $40-49$ & 43 & 30.07 \\
\hline & 50 or above & 71 & 49.65 \\
\hline & I'd rather not say & 0 & 0.00 \\
\hline \multirow[t]{7}{*}{ Highest level of education } & High School or GED & 0 & 0.00 \\
\hline & Some college course work & 3 & 2.10 \\
\hline & Associate degree & 7 & 4.90 \\
\hline & Bachelor's degree & 19 & 13.29 \\
\hline & Master's degree & 95 & 66.43 \\
\hline & Ph.D. or Ed.D. & 7 & 4.90 \\
\hline & Other & 12 & 8.39 \\
\hline \multirow[t]{9}{*}{ Ethnicity } & American Indian or Alaskan Native & 1 & 0.67 \\
\hline & Asian & 1 & 0.67 \\
\hline & Pacific Islander & 1 & 0.67 \\
\hline & Black or African American & 1 & 0.67 \\
\hline & White/Non-Hispanic/Non-Latino & 116 & 77.33 \\
\hline & Hispanic or Latino & 21 & 14.00 \\
\hline & Bi/Multi-racial & 1 & 0.67 \\
\hline & Other & 0 & 0.00 \\
\hline & I'd rather not say & 8 & 5.33 \\
\hline
\end{tabular}

Demographic characteristics. Majority of the leaders ( $n=137,98.8 \%)$ identified themselves as female, were 50 years of age or older $(n=71,49.65 \%)$, leaders identified themselves as white/non-Hispanic/non-Latino $(\mathrm{n}=116,77.33 \%)$, and had master's degree $(n=95,66.43 \%)$. The leaders had the opportunity to write in other educational degrees that they held, and 12 leaders $(8.30 \%)$ stated that they held other educational degrees including education specialist degree. identified themselves as Hispanic or Latino. Please see Table 1 for detailed demographic information. 
Characteristics of the leaders' current leadership position. In order to gain insight into the characteristics of the district-level and county-level EC and ECSE leaders' current position, the leaders responded to questions addressing different aspects of their job. In regard to the agency that the leaders worked for, the majority of the leaders were employed by school districts $(n=110,61.45 \%)$. With this question, the EC and ECSE leaders could add any other agency not among the options. Colorado Department of Education (CDE), private family foundations, local non-profit early childhood programs, and state early childhood councils were the other hiring agencies mentioned by some of the leaders (Table 3 ). 
Table 3

Characteristics of the Participants' Current Leadership Positions

\begin{tabular}{|c|c|c|c|}
\hline Category & Sub-category & $\begin{array}{l}\text { Frequency } \\
(\mathrm{N})\end{array}$ & $\begin{array}{c}\text { Percent } \\
(\%)\end{array}$ \\
\hline Agency & $\begin{array}{l}\text { Colorado Community Board (Early Intervention Program) } \\
\text { School District } \\
\text { Private Preschool } \\
\text { Early Head Start(EHS) } \\
\text { Head Start (HS) } \\
\text { Board of Cooperative Education Services (BOCES) } \\
\text { Other }\end{array}$ & $\begin{array}{r}4 \\
110 \\
8 \\
11 \\
25 \\
11 \\
10\end{array}$ & $\begin{array}{r}2.33 \\
61.45 \\
4.47 \\
6.15 \\
13.97 \\
6.15 \\
5.59\end{array}$ \\
\hline $\begin{array}{l}\text { Administered } \\
\text { programs }\end{array}$ & $\begin{array}{l}\text { Early Intervention Colorado Program (IDEA Part C, Birth-3) } \\
\text { Colorado Preschool Program } \\
\text { Preschool Early Childhood Special Education (IDEA part B, 619) } \\
\text { Child Find } \\
\text { Early Head Start (EHS) } \\
\text { Head Start (HS) }\end{array}$ & $\begin{array}{l}27 \\
83 \\
87 \\
70 \\
15 \\
34\end{array}$ & $\begin{array}{r}8.54 \\
26.27 \\
27.53 \\
22.15 \\
4.75 \\
10.76\end{array}$ \\
\hline Years of experience & $\begin{array}{l}\text { Less than } 5 \text { years } \\
5-9 \text { years } \\
10-14 \text { years } \\
15-19 \text { years } \\
20 \text { years and above }\end{array}$ & $\begin{array}{l}25 \\
33 \\
28 \\
13 \\
44\end{array}$ & $\begin{array}{r}17.48 \\
23.08 \\
19.58 \\
9.09 \\
30.77\end{array}$ \\
\hline $\begin{array}{l}\text { Age range of children } \\
\text { served in the program }\end{array}$ & $\begin{array}{l}\text { Birth- } 3 \text { years of age } \\
\text { Birth- } 5 \text { years of age } \\
3-5 \text { years of age } \\
5-8 \text { years of age } \\
\text { Other }\end{array}$ & $\begin{array}{r}21 \\
58 \\
87 \\
5 \\
11\end{array}$ & $\begin{array}{r}11.54 \\
31.87 \\
47.80 \\
2.75 \\
6.04\end{array}$ \\
\hline $\begin{array}{l}\text { Geographical region in } \\
\text { current position }\end{array}$ & $\begin{array}{l}\text { Urban } \\
\text { Suburban } \\
\text { Rural } \\
\text { Mountain } \\
\text { Frontier } \\
\text { Combination of types } \\
\text { Other }\end{array}$ & $\begin{array}{r}32 \\
32 \\
77 \\
22 \\
2 \\
12 \\
3\end{array}$ & $\begin{array}{r}17.78 \\
17.78 \\
42.78 \\
12.22 \\
1.11 \\
6.67 \\
1.67\end{array}$ \\
\hline $\begin{array}{l}\text { Licensure/endorsement } \\
\text { in current position }\end{array}$ & $\begin{array}{l}\text { K-21 special education generalist license/endorsement } \\
\text { Principal license/endorsement } \\
\text { Early childhood license/endorsement } \\
\text { Early childhood special education license/endorsement } \\
\text { Elementary license/endorsement } \\
\text { CDHS Child Care Director Qualifications } \\
\text { Special Education Director license/endorsement } \\
\text { None } \\
\text { Other }\end{array}$ & $\begin{array}{l}20 \\
32 \\
50 \\
53 \\
29 \\
54 \\
11 \\
15 \\
19\end{array}$ & $\begin{array}{r}7.07 \\
11.31 \\
17.67 \\
18.73 \\
10.25 \\
19.08 \\
3.89 \\
5.30 \\
6.71\end{array}$ \\
\hline $\begin{array}{l}\text { If the leader holds any } \\
\text { other positions } \\
\text { besides leadership } \\
\text { role }\end{array}$ & $\begin{array}{l}\text { Yes } \\
\text { No }\end{array}$ & $\begin{array}{r}43 \\
100\end{array}$ & $\begin{array}{l}30.07 \\
69.93\end{array}$ \\
\hline
\end{tabular}


In terms of the leaders' current position, majority of the leaders $(n=44,30.77 \%)$ had 20 years of experience or above, and served children 3-5 years of age $(n=87$, $47.8 \%$ ). Having the option to state any other age range of children that the leaders might serve, some leaders mentioned serving a specific age range such as 4-5, 12 months and older, or 6-12 age range (Table 3). In terms of the geographical region of the leaders' current position, the majority of leaders $(n=77,42.78 \%)$ worked in rural areas. Three leaders $(1.67 \%)$ added additional information by stating that they worked all over the state, or county-wide (Table 3).

The leaders also stated having different licensure/endorsements for their current position. Fifty-four leaders (19.08\%) reported having Colorado Department of Human Services (CDHS) Child Care Director Qualifications that is relevant to the fields of EC/ECSE. Nineteen leaders added additional information in terms of their licensure/endorsement including a K-21 related service provider license, school social work license, school psychologist license, speech language pathologist license, professional counsellor license, or an administrator license.

In regards to the question that asked the leaders if they held any other positions besides their current leadership role Forty-three leaders (30.07\%) reported having other positions besides their current leadership role such as university instructor, coordinator of services, consultant, preschool directors, mental health coordinator, teacher, or K-12 principal (Table 3).

Leaders' academic and professional characteristics. Table 4 shows the EC and ECSE district-level and county-level leaders' academic and professional characteristics. Ninety-four leaders $(65.73 \%)$ believed that their academic background prepared them for 
the requirements of their current position, while 131 leaders $(91.61 \%)$ believed their professional background prepared them for their leadership responsibilities in their position. Seventy-two leaders $(50.35 \%)$ reported receiving necessary professional development for the requirements of their current position. Seventy-two leaders $(50.35 \%)$ also stated attending professional development activities by national entities, and 134 leaders $(93.7 \%)$ reported attending such professional development activities within the state of Colorado in the past two years. Only 24 leaders $(16.78 \%)$ reported providing professional development activities to state or national level staff in their current position in the past two years. 
Table 4

Participants' Academic and Professional Characteristics

\begin{tabular}{|c|c|c|c|}
\hline Category & Sub-category & $\begin{array}{l}\text { Frequency } \\
(\mathrm{N})\end{array}$ & $\begin{array}{c}\text { Percent } \\
(\%)\end{array}$ \\
\hline \multirow{2}{*}{$\begin{array}{l}\text { If the leader's academic background prepared him/her } \\
\text { for the requirements of the current position. }\end{array}$} & Yes & 94 & 65.73 \\
\hline & No & 49 & 34.27 \\
\hline \multirow{2}{*}{$\begin{array}{l}\text { If the leader's professional background prepared } \\
\text { him/her for the requirements of the current } \\
\text { position. }\end{array}$} & Yes & 131 & 91.61 \\
\hline & No & 12 & 8.39 \\
\hline \multirow{2}{*}{$\begin{array}{l}\text { If the leader has received necessary professional } \\
\text { development for the requirements of the current } \\
\text { position. }\end{array}$} & Yes & 72 & 50.35 \\
\hline & No & 71 & 49.65 \\
\hline \multirow{2}{*}{$\begin{array}{l}\text { If the leader is a member of any national/international } \\
\text { organization. }\end{array}$} & Yes & 67 & 46.85 \\
\hline & No & 76 & 53.15 \\
\hline \multirow{2}{*}{$\begin{array}{l}\text { If the leader has attended any professional } \\
\text { development activity by any national entities in the } \\
\text { past two years. }\end{array}$} & Yes & 72 & 50.35 \\
\hline & No & 71 & 49.65 \\
\hline \multirow{2}{*}{$\begin{array}{l}\text { If the leader has attended any professional } \\
\text { development activity within the state of Colorado } \\
\text { in the past two years. }\end{array}$} & Yes & 134 & 93.7 \\
\hline & No & 9 & 6.29 \\
\hline \multirow{2}{*}{$\begin{array}{l}\text { If the leader has provided any professional } \\
\text { development to state or national level staff in } \\
\text { her/his current position in the past two years. }\end{array}$} & Yes & 24 & 16.78 \\
\hline & No & 119 & 83.22 \\
\hline
\end{tabular}

In terms of leaders' membership in national/international organizations, 67 leaders $(46.85 \%)$ reported such membership with different organizations. The main organizations in the order of the most mentioned include: the National Association for Education of Young Children (NAEYC), the National Head Start Association (NHSA), the CEC, the National Board in Certification for Occupational Therapy (NBCOT), the Council for Advancement and Support of Education (CASE), the DEC, and Zero to Three (Table 4). 


\section{Research Question Two}

Q2 How do district-level and county-level early childhood and early childhood special education leaders evaluate their leadership implementation?

Using the 26 recommended leadership practices, drawn from the Leadership Performance Checklists (ECTA, 2019), the second research question aimed to examine the current state of leadership implementation by district-level and county-level EC and ECSE leaders. The EC/ECSE leaders self-evaluated their implementation of 26 leadership practices using a 4-point Likert scale that included: seldom or never $(0-25 \%)$, some of the time (25-50\%), as often as I can (50-75\%), and most of the time (75-100\%). For the sake of brevity, a list of the top five highest rated implemented practices and low implementation practices was created, which was determined by combining as often as I can $(50-75 \%)$, and most of the time (75-100\%) responses to represent the highest rated and seldom or never (0-25\%) and some of the time (25-50\%) responses to represent the low implementation practices (Table 5). 
Table 5

Highest Rated Leadership Practices in Terms of Leadership Implementation

\begin{tabular}{lcc}
\hline \multicolumn{1}{c}{ Implementation Level - Leadership } & $\begin{array}{c}\text { Count } \\
\text { (n) }\end{array}$ & $\begin{array}{c}\text { Percentage } \\
\text { (\%) }\end{array}$ \\
\hline $\begin{array}{l}\text { High implementation } \\
\text { Create an organizational environment in which all staff members are } \\
\text { treated with respect and trust. }\end{array}$ & 139 & 97.21 \\
$\begin{array}{l}\text { Provide clear information about the purpose and expectations of } \\
\text { assigned tasks or responsibilities. }\end{array}$ & 138 & 96.5 \\
$\begin{array}{l}\text { Participates in developing a program culture that has an inclusive } \\
\text { focus. }\end{array}$ & 134 & 93.7 \\
$\begin{array}{l}\text { Create transparency with open, respectful dialogue and discussion. } \\
\text { Create an organizational culture that values transparency and } \\
\text { collaborative decision making. }\end{array}$ & 134 & 93.7 \\
$\begin{array}{l}\text { Low implementation } \\
\text { Engage in planning and conducting cross-agency training and staff } \\
\text { development opportunities. }\end{array}$ & 129 & 90.21 \\
$\begin{array}{l}\text { Continue to learn and stay abreast of knowledge and research pertinent } \\
\text { to work and share this information with other colleagues. }\end{array}$ & 64 & 44.75 \\
$\begin{array}{l}\text { Seek and support opportunities to work in partnership with other } \\
\text { agency and program leaders to promote services and supports for all } \\
\text { children and families. }\end{array}$ & 41 & 30.07 \\
$\quad \begin{array}{l}\text { Understand other programs' and agencies' missions, visions, goals, } \\
\text { and the services and supports they provide. }\end{array}$ & 36 & 28.67 \\
$\begin{array}{l}\text { Communicate statutes, policies, codes of ethics, and procedures to } \\
\text { assist others in understanding the reasons behind decisions and } \\
\text { actions. }\end{array}$ & 34 & 25.17 \\
\hline
\end{tabular}

Table 5 reports the top five highest rated leadership practices in terms of high and low implementation. Treating staff with respect and trust $(\mathrm{n}=139,97.21 \%)$ included the highest implementation and planning and conducting cross-agency staff development (n $=64,44.75 \%$ ) included the lowest implementation among the leaders.

\section{Research Questions Three}

Q3 What challenges do these leaders encounter in leadership implementation and development in their programs?

The third research question aimed to examine the challenges that district-level and county-level EC and ECSE leaders encounter in leadership implementation for each of the 26 recommended leadership practices. The EC/ECSE leaders used a 4-point Likert 
scale that included: not challenging, somewhat challenging, challenging, and very challenging to rate the implementation challenges for reach practice. Similar to Research Question 2, for the sake of brevity, a list of the top five highest rated leadership practices in terms of high and low implementation challenges was created, combining the challenging and very challenging responses to represent highest rated in terms of high implementation challenges and not challenging and somewhat challenging responses to represent highest rated in terms of low implementation challenges (Table 6). 
Table 6

Highest Rated Leadership Practices in Terms of Implementation Challenges

\begin{tabular}{lcc}
\hline \multicolumn{1}{c}{ Levels of Implementation - Challenges } & $\begin{array}{c}\text { Count } \\
(\mathrm{n})\end{array}$ & $\begin{array}{c}\text { Percentage } \\
(\%)\end{array}$ \\
\hline $\begin{array}{l}\text { High challenges } \\
\quad \begin{array}{l}\text { Advocate for and secure the fiscal and human resources needed to } \\
\text { provide quality services and supports. }\end{array}\end{array}$ & 92 & 64.33 \\
$\quad \begin{array}{l}\text { Understand and communicate how your program/agency fits into the } \\
\text { larger service system. }\end{array}$ & 70 & 48.96 \\
$\begin{array}{l}\text { Establish empowering relationships and collaborative partnership with } \\
\text { families. }\end{array}$ & 69 & 48.26 \\
$\begin{array}{l}\text { Engage in planning and conducting cross-agency training and staff } \\
\text { development opportunities. }\end{array}$ & 68 & 47.55 \\
$\begin{array}{l}\text { Advocate and develop strategies for cultural diversity and modify } \\
\text { interventions and practices accordingly. }\end{array}$ & 66 & 46.12 \\
$\begin{array}{l}\text { Low challenges } \\
\text { Provide clear information about the purpose and expectations of } \\
\text { assigned tasks or responsibilities. }\end{array}$ & 100 & 69.93 \\
$\quad \begin{array}{l}\text { Understand and establish professional boundaries; yet promote an open } \\
\text { and caring workplace where people want to come each day. }\end{array}$ & 100 & 69.93 \\
$\begin{array}{l}\text { Create an organizational environment in which all staff members are } \\
\text { treated with respect and trust. }\end{array}$ & 98 & 68.53 \\
$\quad \begin{array}{l}\text { Understand other programs' and agencies' missions, visions, goals, and } \\
\text { the services and supports they provide. }\end{array}$ & 98 & 68.53 \\
$\begin{array}{l}\text { Advocate for and promote the importance of early intervention and } \\
\text { early childhood services and supports for all children and families. }\end{array}$ & 94 & 65.73 \\
\hline
\end{tabular}

Table 6 shows the top five highest rated recommended leadership practices in terms of highest and lowest levels of PD needs in implementation of the leadership practices. According to Table 6, securing fiscal and human resources $(\mathrm{n}=92,64.33 \%)$ was regarded as the most challenging leadership practice to implement and providing clear information about the expectations and responsibilities $(\mathrm{n}=100,69.93 \%)$ regarded as the least challenging leadership practice in terms of implementation.

\section{Research Question Four}

Q4 What professional development and support do these leaders need to implement quality leadership in their practice? 
The fourth research question aimed to examine the levels of the professional development need that district-level and county-level EC and ECSE leaders have in implementation of the 26 recommended leadership practices. The EC/ECSE leaders rated the levels of the professional development needs in implementation of the recommended leadership practices using a 4-point Likert scale that included: low professional development need, medium professional development need, high professional development need, and very high professional development need. Similar to Research Questions 2 and 3, for the sake of brevity, a list of the top five highest rated leadership practices in terms of high and low levels of professional development need was created by combining high professional development need and very high professional development need to represent highest rated in terms of high levels of professional development needs and medium professional development need and low professional development need responses to represent highest rated in terms of low levels of professional development needs (Table 7). 
Table 7

Highest Rated Leadership Practices in Terms of Professional Development Needs in Implementation of Leadership Practices

Levels of Leadership Practices - Professional Development Need

Count

Percentage

(n)

$(\%)$

\begin{tabular}{|c|c|c|}
\hline \multicolumn{3}{|l|}{ High levels } \\
\hline $\begin{array}{l}\text { Advocate for and secure the fiscal and human resources needed to } \\
\text { provide quality services and supports. }\end{array}$ & 71 & 49.65 \\
\hline $\begin{array}{l}\text { Advocate and develop strategies for cultural diversity and modify } \\
\text { interventions and practices accordingly. }\end{array}$ & 70 & 48.95 \\
\hline Participates in developing a program culture that has an inclusive focus. & 65 & 45.45 \\
\hline $\begin{array}{l}\text { Establish empowering relationships and collaborative partnership with } \\
\text { families. }\end{array}$ & 63 & 44.05 \\
\hline $\begin{array}{l}\text { Develop priorities and strategic plans consistent with the vision and } \\
\text { mission. }\end{array}$ & 60 & 41.96 \\
\hline \multicolumn{3}{|l|}{ Low levels } \\
\hline $\begin{array}{l}\text { Establish working relationships with colleagues, beyond attending } \\
\text { formal meetings. }\end{array}$ & 128 & 89.51 \\
\hline $\begin{array}{l}\text { Provide clear information about the purpose and expectations of } \\
\text { assigned tasks or responsibilities. }\end{array}$ & 115 & 80.42 \\
\hline $\begin{array}{l}\text { Understand and establish professional boundaries; yet promote an open } \\
\text { and caring workplace where people want to come each day. }\end{array}$ & 115 & 80.42 \\
\hline $\begin{array}{l}\text { Create an organizational environment in which all staff members are } \\
\text { treated with respect and trust. }\end{array}$ & 108 & 75.52 \\
\hline $\begin{array}{l}\text { Ensure that staff members take individual responsibility and honor the } \\
\text { responsibilities of others for getting work done in a competent and } \\
\text { timely way. }\end{array}$ & 108 & 75.52 \\
\hline
\end{tabular}

Table 7 shows the top five highest rated recommended leadership practices in terms of high and low levels of the professional development. Securing fiscal and human resources $(\mathrm{n}=71,49.65 \% 0)$ was the highest rated leadership practice in terms of high levels of PD and establishing working relationships with colleagues $(\mathrm{n}=128,89.51 \%)$ was the highest rated leadership practice in terms of the low levels of PD among the leaders. 


\section{Survey Section 3: Open-ended Questions}

The third section of the survey included five open-ended questions that asked participants about additional comments around their leadership implementation and evaluation, their challenges, professional development needs, and other contributing factors to facilitate quality leadership in their programs. The open-ended questions that were used in the third section of the survey included:

1. Do you conduct any evaluations of leadership practices for yourself or for others in your program? Please explain and provide examples.

2. Please state any other comments you have regarding leadership implementation in your practice/program in general that we have not addressed in the questionnaire.

3. Please state any other challenges you regularly encounter in your role as an EC/ECSE leader that we have not addressed in the questionnaire.

4. Please state any other professional development needs you have in your current role as an EC/ECSE leader that we have not addressed in the questionnaire. What else do you think can make a considerable contribution to leadership development and sustainability in EC/ECSE in Colorado?

5. Any additional comments?

Below, the findings from each question are summarized. Table 8 includes a summary of the responses to the open-ended questions. 
Table 8

Summary of the Responses to the Open-ended Questions

\begin{tabular}{|c|c|c|c|}
\hline Areas & Themes & $\begin{array}{c}\text { Counts } \\
(n)\end{array}$ & $\begin{array}{l}\text { Percentage } \\
(\%)\end{array}$ \\
\hline \multirow{9}{*}{$\begin{array}{l}\text { Leadership } \\
\text { Evaluation }\end{array}$} & No evaluations & 15 & 25.8 \\
\hline & $\begin{array}{l}\text { Annual surveys from their agency and or administrative unit using tools such } \\
\text { as Quality Rating Improvement, Colorado Model Performance } \\
\text { Management }\end{array}$ & 13 & 22.4 \\
\hline & Collaborative reflections with peers & 8 & 13.7 \\
\hline & Self-reflection and self-analysis & 6 & 10.3 \\
\hline & On-site visits form their agency and/or administrative unit & 5 & 8.7 \\
\hline & State evaluation by the school district & 5 & 8.7 \\
\hline & Using professional competencies' checklist & 3 & 5.2 \\
\hline & Evaluations using self-created tools & 3 & 5.2 \\
\hline & Total & 58 & 100 \\
\hline \multicolumn{4}{|l|}{ Leadership } \\
\hline \multirow[t]{11}{*}{ Implementation } & Difficulty matching needs to professional development programs & 13 & 20.6 \\
\hline & $\begin{array}{l}\text { Many rules and entities involved in inclusive programs (Head Start, Colorado } \\
\text { Preschool Program, Child Care Licensing, and school district policy) }\end{array}$ & 11 & 17.4 \\
\hline & The role is more about supervision and management not leadership & 9 & 14.2 \\
\hline & $\begin{array}{l}\text { Very few leaders with in-depth knowledge regarding early childhood } \\
\text { specifically }\end{array}$ & 7 & 11.2 \\
\hline & Need for an evidence-based framework for leadership in EC education & 5 & 7.9 \\
\hline & Need greater opportunities for distributed leadership & 5 & 7.9 \\
\hline & Old-fashion view toward inclusion & 4 & 6.4 \\
\hline & $\begin{array}{l}\text { Need education and training on teaming and collaboration between general } \\
\text { education and special education staff }\end{array}$ & 4 & 6.4 \\
\hline & The leadership role mostly includes secretarial level work & 3 & 4.8 \\
\hline & Not having decision making authority. & 2 & 3.2 \\
\hline & Total & 63 & \\
\hline \multicolumn{4}{|l|}{ Leadership } \\
\hline \multirow[t]{17}{*}{ Challenges } & Wearing too many hats as a "one-woman show" & 12 & 19.6 \\
\hline & Big division between $\mathrm{EC}$ and ECSE & 7 & 11.5 \\
\hline & Lack of understanding of the importance of EC education and inclusion & 6 & 9.6 \\
\hline & High staff turnover and issues to recruit and retain quality staff & 5 & 8.3 \\
\hline & Funding in rural districts & 4 & 6.6 \\
\hline & Lack of time to solve problems collaboratively & 4 & 6.6 \\
\hline & Creating a culture of trust among staff & 3 & 4.9 \\
\hline & $\begin{array}{l}\text { The never-ending mandates (e.g., Colorado Shines, CLASS, ECERS, } \\
\text { Teaching Strategies GOLD, etc.) }\end{array}$ & 3 & 4.9 \\
\hline & Laws that don't always support the children and family & 3 & 4.9 \\
\hline & Communication & 2 & 3.3 \\
\hline & Lack of human resources in rural districts & 2 & 3.3 \\
\hline & Partnering with families & 2 & 3.3 \\
\hline & Poor facilities and inefficient learning environment & 2 & 3.3 \\
\hline & Compliance with IDEA & 2 & 3.3 \\
\hline & Not knowing what resources/books/tools are out and available to use & 2 & 3.3 \\
\hline & Overwhelming paperwork for licensure & 2 & 3.3 \\
\hline & Total & 61 & \\
\hline
\end{tabular}


Table 8 (continued)

\begin{tabular}{|c|c|c|c|}
\hline Areas & Themes & $\begin{array}{c}\text { Counts } \\
(\mathrm{n})\end{array}$ & $\begin{array}{c}\text { Percentage } \\
(\%)\end{array}$ \\
\hline \multicolumn{3}{|l|}{ Professional } & \\
\hline \multirow[t]{10}{*}{ Needs } & The need for professional development around EC mental health & 14 & 24.2 \\
\hline & Funding for recruitment, retention and compensation of staff & 9 & 15.6 \\
\hline & Cross-agency collaboration & 9 & 15.6 \\
\hline & Effective leadership models specific to EC context & 7 & 12.2 \\
\hline & Coaching workshops & 5 & 8.6 \\
\hline & $\begin{array}{l}\text { Collaboration between Colorado Department of Education and Colorado } \\
\text { Department of Human Services }\end{array}$ & 4 & 6.8 \\
\hline & Compliance with IDEA requirements & 4 & 6.8 \\
\hline & Systemic racism & 3 & 5.1 \\
\hline & How to manage resources in meaningful ways & 3 & 5.1 \\
\hline & Total & 58 & 100 \\
\hline \multicolumn{4}{|l|}{ Additional } \\
\hline \multirow[t]{5}{*}{ Comments } & The need to build cooperation and positive culture among staff & 4 & 40 \\
\hline & More responsibilities and less energy for children. & 3 & 30 \\
\hline & $\begin{array}{l}\text { The need for core values about promoting developmentally appropriate } \\
\text { community and early childhood systems }\end{array}$ & 2 & 20 \\
\hline & The need to build out attitudes toward parents and families & 1 & 10 \\
\hline & Total & 10 & 100 \\
\hline
\end{tabular}

Open-ended Question 1: Leadership evaluation. The first open-ended question asked the leaders if they conduct any evaluations of leadership practices in their program. Fifty-eight leaders responded to this question. Specific themes and number of occurrences are presented in Table 8 . The majority of the leaders reported not conducting any evaluations $(\mathrm{n}=15,25.8 \%)$. Some leaders conducted evaluations and stated using surveys provided by their agencies or administrative units $(n=13,22.4 \%)$. Some leaders also reported using collaborative reflections with their peers $(n=8,13.7 \%)$ or selfreflections $(n=6,10.3 \%)$ to self-analyze their leadership performance.

Open-ended Question 2: Leadership implementation. The second open-ended question asked participants to reflect on their leadership implementation. Sixty-three leaders responded to this question. The major theme involved the need for providing relevant professional development opportunities for both leaders and $\operatorname{staff}(\mathrm{n}=13$, 20.6\%). The leaders stated that there is a great mismatch between what they themselves, 
their staff, and their programs needed and the available resources from local and state entities that are provided for relevant professional development.

Also, in terms of inclusive programs, many leaders $(\mathrm{n}=11,17.4 \%)$ stated having difficulties to maintain quality programming due to the many rules form different agencies and entities such as HS, CPP, Child Care Licensing, the IDEA, and school district policies that make the interpretation of the rules and regulations different among the different agencies. Some of the leaders $(n=9,14.2 \%)$ also commented on the nature of their positions, which included more of supervision and management and not necessarily leadership. They saw their staff generally following their direction as the supervisors in the workplace which, according to them, doesn't make them "leaders." They thought they needed better leadership skills, competencies, and opportunities so that their staff were able to follow them as leaders to move the work forward. The other frequently mentioned theme $(\mathrm{n}=7,11.2 \%)$ was in regard to lack of strong knowledge base of the EC/ECSE leaders. According to these leaders, many of them were not trained in EC practices and there seemed to be not enough thought about who is being put in the leadership roles for EC programs. The leaders stated the need for more leaders who feel confident and competent about implementing EC practices at the program level. The need for an evidence-based framework specific to EC education and setting for the leaders to follow, the need for more distributed leadership, and more teaming and collaboration were among other mentioned themes. Please see Table 9 for a complete list of the themes.

Open-ended Question 3: Leadership challenges. The third open-ended question asked participants about their challenges in implementation of quality leadership 
practices. Sixty-one leaders responded to this question. The main theme that many of the leaders mentioned $(\mathrm{n}=12,19.6 \%)$ included wearing many different hats in different positions specifically in rural areas that included overseeing special education and general EC services, working with finance, choice and enrollment, tuition and billing, curriculum and instruction, research and evaluation, and human resources. According to these leaders, this usually leads to difficulties in balancing the many responsibilities and investing more on quality services and more professional development in the field.

Some of the leaders $(\mathrm{n}=7,11.5 \%)$ also believed that there is a big division between EC and ECSE that lends itself to miscommunication, lack of collaboration, and different team dynamics. According to the leaders, this concern also results in lack of collaboration and teaming among the staff from the fields or EC and ECSE and, therefore, lack of efficiency and efficacy of instructional outcomes specifically around inclusive practices. The EC and ECSE leaders $(n=6,9.6 \%)$ also commented on the challenges they face to get others in the field to see and admit the benefit of early childhood. According to these leaders, they were having difficulties convincing others (e.g., school board members) that EC education is a vital piece in a child's education. They thought that they were being considered as "glorified babysitters" and all they did with children was play. Some of the leaders also commented that they had invited stakeholders to their center to observe and explain the science behind their teaching methods. However, according to these leaders, they were still not being taken seriously. Some of the leaders ( $\mathrm{n}=5,8.3 \%$ ) also mentioned high turnover in the field, especially among preschool teachers that was a result of poor compensations and lack of facilities and confusion about what the staff and teachers should be paid for (e.g., if they are 
supposed to be paid for professional development). Lack of funding and time for collaborative problem solving and too many mandates, rules and regulations to follow in their positions were among the other mentioned themes. Please see Table 8 for a complete list of themes in terms of challenges in implementation of recommended leadership practices.

Open-ended Question 4: Professional development needs. The fourth openended question asked the leaders to comment about their professional development needs. Fifty-eight leaders responded to this question. The most frequently mentioned theme was about more professional development around EC mental health $(\mathrm{n}=14,24.2 \%)$. These leaders stated the need for early childhood mental health professionals such as social workers and counselors who have an educational pathway to gain the expertise necessary to work with parents of children birth to school age in EC settings. The leaders commented that many of them have difficulties with hiring well-trained professionals in areas related to mental health. One consequence, according to some of these leaders in rural areas, was having to send their professionals to very expensive trainings and when the professionals are trained and experienced, they move away from rural areas. Some of the leaders $(\mathrm{n}=9,15.6 \%)$ also stated funding for quality professional development as their main concern. According to these leaders, when there is a good training opportunity, funding is difficult, especially in rural districts. These leaders mentioned their needs for more funded state-level professional development as the local opportunities typically cover topics they already are familiar with.

Better coordination of cross-agency collaboration was another theme that EC and ECSE leaders mentioned $(n=9,15.6 \%)$. These leaders stated the need for actual 
collaboration and support, rather than a list of what each one does. The other theme was related to the leaders' need to learn more about effective hiring processes and intentional hiring/orientation processes that result in long-term retention of an EC workforce. The leaders stated the need to learn about strategies beyond increasing living wages for early childhood educators to recruit and retain a high-quality early childhood workforce.

Some of the leaders ( $\mathrm{n}=7,12.2 \%)$ also mentioned the need for a specific degree program, certification or license that focuses solely on EC/ECSE leadership. They believed they needed to learn more about effective leadership models specific to the EC context as many of them come from different backgrounds and with different qualifications. According to these leaders, there is not a system in the state that really addresses the professional development needs for EC/ECSE leaders in terms of administrative leadership. Most of the professional development opportunities are for teaching and child care professionals, not program leaders. Table 8 provides a complete list of themes in regard to the leaders' comments around their professional development needs.

Open-ended Question 5: Additional comments. The fifth open-ended question asked for the leaders' additional comments in general. Only 10 leaders added additional comments that have been already covered among the aforementioned themes (Table 8).

\section{Phase I: Quantitative Data--Secondary Data Analysis Results}

As outlined in Chapter III, a three-step inferential statistical analysis was conducted to reach conclusions that extend beyond the immediate data alone and make inferences from the data to more general conditions. Using Statistical Package for the Social Sciences (SPSS), first, Cronbach's Alpha was computed to inform the internal 
consistency of the measure and reliability of the scores. Second, a bivariate correlations and exploratory factor analysis (EFA) was conducted to gain a better understanding of the underlying constructs in each of the three main scales of the survey including implementation of the recommended leadership practices, implementation challenges, and levels of professional development need in implementation of recommended leadership practices. Third, a multivariate analysis of variance (MANOVA) was conducted to determine whether there were any statistically significant differences between the means of the groups and if a certain demographic characteristic explained the differences.

The possible score range for the survey was between 26 and 104 based on summing the responses to the 26 recommended leadership practices. The procedure for scoring the survey for the purpose of inferential data analysis included summing the scores of the 26 recommended leadership practices in each of the three scales (implementation of recommended leadership practices, challenges in implementation of the recommended leadership practices, and level of professional development in implementation of recommended leadership practices) and using each of the summed scores as one dependent variable (DV) in the statistical analysis. In what follows, each of the steps are presented in more detail.

\section{Reliability Analysis}

Reliability in statistics and psychometrics is the overall consistency of a measure (Rosenblad, 2009). A measure is said to have high reliability if it produces similar results under consistent conditions. Scores that are highly reliable are accurate, reproducible, and consistent from one testing occasion to another (Scherpenzeel \& Saris, 1997). The 
analysis of reliability is called reliability analysis. Reliability analysis is determined by obtaining the proportion of systematic variation in a scale, which can be done by determining the association between the scores obtained from different administrations of the scale (Scherpenzeel \& Saris, 1997). Thus, if the association in reliability analysis is high, the scale yields consistent results and is, therefore, reliable (Rosenblad, 2009).

Cronbach's alpha is a measure used to assess the reliability or the strength of the internal consistency of the scores produced by a set of scales or test items (Rosenblad, 2009). The resulting Alpha coefficient of reliability ranges from 0 to 1 in providing the overall assessment of a measure's reliability of scores. If all of the scale items are entirely independent from one another, (i.e., the items are not correlated or share no covariance), the alpha equals 0 . If all of the items are correlated and have high covariance, alpha will approach 1. In other words, the higher the alpha coefficient, the more the items have shared covariance and probably measure the same underlying concept. A reliability coefficient of 0.7 or higher is considered "acceptable" in most social science research situations (Rosenblad, 2009).

Cronbach's alpha was computed for each of the three scales to estimate the internal consistency and reliability of scores. A Cronbach's alpha of 0.911 for the implementation of recommended leadership practices, a Cronbach's alpha of 0.910 for challenges in implementation of the recommended leadership practices, and a Cronbach's alpha of 0.924 for the levels of the professional development need in implementation of recommended leadership practices were obtained. This indicates that the items in each of the three scales have relatively high internal consistency and produce reliable scores. Please see Table 9 for more details of the reliability and scale statistics. 
Table 9

Reliability and Scale Statistics

\begin{tabular}{cccccc}
\hline & Reliability Statistics & \multicolumn{3}{c}{ Scale Statistics } \\
\cline { 2 - 6 } Implementation & $\begin{array}{c}\text { Cronbach's } \\
\text { Alpha }\end{array}$ & Mean & Variance & SD & $\begin{array}{c}\text { Items } \\
\text { (N) }\end{array}$ \\
\hline Leadership implementation & .911 & 82.17 & 129.342 & 11.878 & 26 \\
$\begin{array}{c}\text { Leadership implementation } \\
\text { challenges }\end{array}$ & .910 & 58.78 & 137.312 & 11.718 & 26 \\
$\begin{array}{c}\text { Professional development } \\
\text { needs in leadership } \\
\text { implementation }\end{array}$ & .924 & 53.29 & 171.899 & 13.111 & 26 \\
\hline
\end{tabular}

\section{Exploratory Factor Analysis}

Bivariate correlations and EFA were conducted to identify interpretable factors and underlying constructs among the 26 recommended leadership practices in each of the three scales (implementation of recommended leadership practices, challenges in implementation of the recommended leadership practices, and level of professional development in implementation of recommended leadership practices). Exploratory factor analysis is a statistical approach for determining patterns of correlations among the variables in a dataset (Tabachnick \& Fidell, 2013). The basic assumption of factor analysis is that for a collection of observed variables, there are sets of underlying latent variables called factors that can explain the interrelationships among those variables. Factor analysis tries to achieve parsimony by explaining the maximum amount of common variance in data using the smallest number of explanatory constructs. These explanatory constructs are known as "factors" (or latent variables) as they represent cluster variables that correlate highly with each other (Tabachnick \& Fidell, 2013).

The EFA was run using the SPSS dimension reduction feature. In each of the three scales, the summed score of the 26 recommended leadership practices was chosen 
as the DV. Principal component analysis was chosen as the extraction method, and 0.3 was chosen as the salient pattern of coefficient to determine an item's loading on particular factors with promax as the method of rotation. Below, the results of the EFA for each scale is presented.

Implementation of recommended leadership practices. The EFA for this scale resulted in identification of five components, cumulatively explaining $63 \%$ of the variation in the data. The three first components explained half of the variation $(51.2 \%)$ in the data and were considered as the main factors (see Tables 10 and 11). Considering the loadings of $0.5>$ in the three first components, the factors were representative of visionary leadership development, staff involvement, and cross-agency partnership and support. Table 11 shows the pattern matrix of the extracted loadings. For specific leadership practices, see Appendix A.

Table 10

Extraction of Sums of Squared Loadings for Implementation of Leadership Practices

\begin{tabular}{|c|c|c|c|c|c|c|}
\hline \multirow[b]{2}{*}{ Component } & \multicolumn{3}{|c|}{ Initial Eigenvalues } & \multicolumn{3}{|c|}{ Extraction Sums of Squared Loadings } \\
\hline & $\begin{array}{c}\text { Total } \\
\%\end{array}$ & $\begin{array}{c}\text { Variance } \\
\%\end{array}$ & $\begin{array}{c}\text { Cumulative } \\
\%\end{array}$ & $\begin{array}{c}\text { Total } \\
\%\end{array}$ & $\begin{array}{c}\text { Variance } \\
\%\end{array}$ & $\begin{array}{c}\text { Cumulative } \\
\%\end{array}$ \\
\hline 1 & 8.479 & 32.613 & 32.613 . & 8.479 & 32.613 & 32.613 \\
\hline 2 & 3.005 & 11.557 & 44.170 & 3.005 & 11.557 & 44.170 \\
\hline 3 & 1.832 & 7.045 & 51.215 & 1.832 & 7.045 & 51.215 \\
\hline 4 & 1.799 & 6.920 & 58.136 & 1.799 & 6.920 & 58.136 \\
\hline 5 & 1.326 & 5.101 & 63.237 & 1.326 & 5.101 & 63.237 \\
\hline
\end{tabular}


Table 11

Pattern Matrix of the Extracted Loadings for Implementation of the Recommended Leadership Practices, Phase I Online Survey

\begin{tabular}{|c|c|c|c|c|c|}
\hline \multirow{2}{*}{$\begin{array}{c}\text { Leadership } \\
\text { Practices }\end{array}$} & \multicolumn{5}{|c|}{ Components } \\
\hline & 1 & 2 & 3 & 4 & 5 \\
\hline 8 & $.874^{\mathrm{a}}$ & & & & \\
\hline 7 & $.856^{\mathrm{a}}$ & & & & \\
\hline 20 & $.717^{\mathrm{a}}$ & & & & \\
\hline 4 & $.691^{\mathrm{a}}$ & & & -.317 & \\
\hline 15 & $.595^{\mathrm{a}}$ & & & & \\
\hline 2 & $.591^{\mathrm{a}}$ & & & .307 & \\
\hline 22 & $.529^{\mathrm{a}}$ & & & & \\
\hline 23 & .456 & & & & .355 \\
\hline 10 & -.301 & $.877^{\mathrm{b}}$ & & & \\
\hline 14 & & $.803^{b}$ & & & \\
\hline 21 & & $.685^{\mathrm{b}}$ & & & \\
\hline 11 & & $.655^{\mathrm{b}}$ & .314 & & \\
\hline 13 & & $.613^{\mathrm{b}}$ & & & \\
\hline 12 & & $.501^{\mathrm{b}}$ & & & \\
\hline 25 & & & $.830^{\mathrm{c}}$ & & \\
\hline 24 & & & $.801^{\mathrm{c}}$ & & \\
\hline 19 & & & $.661^{\mathrm{c}}$ & & \\
\hline 6 & & & $.525^{\mathrm{c}}$ & & -.301 \\
\hline 3 & & & & .908 & \\
\hline 1 & & & .353 & .681 & \\
\hline 18 & & .362 & & .507 & \\
\hline 5 & .394 & & & .484 & \\
\hline 26 & & & & & .768 \\
\hline 17 & & & .445 & .313 & .694 \\
\hline 9 & & .318 & & .356 & -.526 \\
\hline 16 & & .392 & & & .469 \\
\hline
\end{tabular}

${ }^{a}$ Visionary leadership development. ${ }^{b}$ Staff involvement and support. ${ }^{c}$ Cross-agency partnership and collaboration.

Challenges in implementation of recommended leadership practices. The

EFA for this scale revealed five components, and the first four components explained half of the variation (51\%) in the data (see Tables 12 and 13). Considering the loadings of $0.5>$ in the first four components, the main factors were representative of transparency and collaborative decision making, empowering relationships with stakeholders, strategic leadership development, and advocacy leadership and staff support. It should be mentioned that in components where there were fewer than four items with loadings of $0.5>$, the items with loadings of $.05<$ were also considered, as to be able to interpret a 
factor in a component, there must be at least four items in that components (Tabachnick \& Fidell, 2013). Table 13 shows the pattern matrix of the extracted loadings. For specific leadership practices, refer to Appendix A.

\section{Table 12}

Extraction of Sums of Squared Loadings for Challenges in Implementation of the Recommended Leadership Practices

\begin{tabular}{|c|c|c|c|c|c|c|}
\hline \multirow[b]{2}{*}{ Component } & \multicolumn{3}{|c|}{ Initial Eigenvalues } & \multicolumn{3}{|c|}{ Extraction Sums of Squared Loadings } \\
\hline & $\begin{array}{c}\text { Total } \\
\% \\
\end{array}$ & $\begin{array}{c}\text { Variance } \\
\% \\
\end{array}$ & $\begin{array}{c}\text { Cumulative } \\
\% \\
\end{array}$ & $\begin{array}{c}\text { Total } \\
\% \\
\end{array}$ & $\begin{array}{c}\text { Variance } \\
\% \\
\end{array}$ & $\begin{array}{c}\text { Cumulative } \\
\%\end{array}$ \\
\hline 1 & 8.280 & 31.847 & 31.847 & 8.280 & 31.847 & 31.847 \\
\hline 2 & 1.846 & 7.099 & 38.946 & 1.846 & 7.099 & 38.946 \\
\hline 3 & 1.752 & 6.739 & 45.685 & 1.752 & 6.739 & 45.685 \\
\hline 4 & 1.383 & 5.318 & 51.003 & 1.383 & 5.318 & 51.003 \\
\hline 5 & 1.310 & 5.040 & 56.043 & 1.310 & 5.040 & 56.043 \\
\hline
\end{tabular}


Table 13

Pattern Matrix of the Extracted Loadings for Challenges in Implementation of the Recommended Leadership Practices, Phase I Online Survey

\begin{tabular}{|c|c|c|c|c|c|}
\hline \multirow{2}{*}{$\begin{array}{c}\text { Leadership } \\
\text { Practices }\end{array}$} & \multicolumn{5}{|c|}{ Component } \\
\hline & 1 & 2 & 3 & 4 & 5 \\
\hline 16 & $.991^{\mathrm{a}}$ & & & & \\
\hline 3 & $.651^{\mathrm{a}}$ & & & & \\
\hline 11 & $.497^{\mathrm{a}}$ & & & & \\
\hline 21 & $.488^{\mathrm{a}}$ & .350 & & & \\
\hline 9 & .466 & & & & \\
\hline 22 & .427 & & & .343 & \\
\hline 15 & .385 & .354 & & & \\
\hline 20 & & $.794^{\mathrm{b}}$ & & & \\
\hline 26 & & $.765^{\mathrm{b}}$ & & & \\
\hline 1 & -.507 & $.751^{\mathrm{b}}$ & & & \\
\hline 23 & & $.637^{\mathrm{b}}$ & & & \\
\hline 12 & & $.558^{\mathrm{b}}$ & .330 & & \\
\hline 18 & & .451 & & .379 & \\
\hline 4 & & & $.815^{\mathrm{c}}$ & & \\
\hline 6 & & & $.746^{\mathrm{c}}$ & & \\
\hline 13 & & & $.661^{\mathrm{c}}$ & & \\
\hline 25 & & & $.612^{\mathrm{c}}$ & & \\
\hline 17 & & & .321 & & \\
\hline 14 & & & & $.723^{\mathrm{d}}$ & \\
\hline 24 & & & .465 & $.705^{\mathrm{d}}$ & \\
\hline 19 & & & .405 & $.551^{\mathrm{d}}$ & \\
\hline 10 & .316 & .304 & & & \\
\hline 8 & & & & $.329^{\mathrm{d}}$ & \\
\hline 2 & & & & & .881 \\
\hline 5 & & & & & .803 \\
\hline 7 & & & & & .465 \\
\hline
\end{tabular}

\section{Levels of the professional development need in implementation of the}

recommended Leadership practices. For this scale, the results of the EFA showed five main components, with the first three components explaining half of the variation in the data $(51 \%)$ (see Tables 14 and 15 for more details. The three main factors represented staff development and support, program sustainability, and visionary leadership development. Table 15 shows the pattern matrix of the extracted loadings. For specific leadership practices, refer to Appendix A. 
Table 14

Extraction of Sums of Squared Loadings for the Levels of the Professional Development Need

\begin{tabular}{|c|c|c|c|c|c|c|}
\hline \multirow[b]{2}{*}{ Component } & \multicolumn{3}{|c|}{ Initial Eigenvalues } & \multicolumn{3}{|c|}{ Extraction Sums of Squared Loadings } \\
\hline & $\begin{array}{c}\text { Total } \\
\% \\
\end{array}$ & $\begin{array}{c}\text { Variance } \\
\%\end{array}$ & $\begin{array}{c}\text { Cumulative } \\
\%\end{array}$ & $\begin{array}{c}\text { Total } \\
\% \\
\end{array}$ & $\begin{array}{c}\text { Variance } \\
\%\end{array}$ & $\begin{array}{c}\text { Cumulative } \\
\%\end{array}$ \\
\hline 1 & 9.249 & 35.573 & 35.573 & 9.249 & 35.573 & 35.573 \\
\hline 2 & 2.344 & 9.016 & 44.589 & 2.344 & 9.016 & 44.589 \\
\hline 3 & 1.771 & 6.811 & 51.400 & 1.771 & 6.811 & 51.400 \\
\hline 4 & 1.609 & 6.190 & 57.590 & 1.609 & 6.190 & 57.590 \\
\hline 5 & 1.337 & 5.144 & 62.734 & 1.337 & 5.144 & 62.734 \\
\hline
\end{tabular}


Table 15

Pattern Matrix of the Extracted Loadings for Levels of Professional Development Needs in Implementation of Recommended Leadership Practices, Phase I Online Survey

\begin{tabular}{|c|c|c|c|c|c|}
\hline \multirow{2}{*}{$\begin{array}{c}\text { Leadership } \\
\text { Practices }\end{array}$} & \multicolumn{5}{|c|}{ Component } \\
\hline & 1 & 2 & 3 & 4 & 5 \\
\hline 16 & $.815^{\mathrm{a}}$ & & & & \\
\hline 15 & $.749^{\mathrm{a}}$ & & & & \\
\hline 10 & $.623^{\mathrm{a}}$ & -.386 & & & \\
\hline 21 & $.483^{\mathrm{a}}$ & .361 & & & \\
\hline 7 & .357 & & & .357 & \\
\hline 9 & & & & & \\
\hline 26 & & $.876^{\mathrm{b}}$ & & & \\
\hline 14 & & $.612^{\mathrm{b}}$ & & & \\
\hline 18 & & $.474^{\mathrm{b}}$ & & & \\
\hline 4 & & $.452^{\mathrm{b}}$ & & & \\
\hline 20 & .418 & .425 & & & \\
\hline 22 & .389 & .420 & .305 & & \\
\hline 2 & & & $.877^{\mathrm{c}}$ & & \\
\hline 1 & & & $.735^{\mathrm{c}}$ & & \\
\hline 5 & -.518 & & $.693^{\mathrm{c}}$ & & .451 \\
\hline 3 & .430 & & $.603^{\mathrm{c}}$ & & \\
\hline 8 & & & .413 & & \\
\hline 24 & & & & .874 & \\
\hline 25 & & & & .749 & \\
\hline 19 & & & & .642 & \\
\hline 6 & & & .419 & .522 & \\
\hline 12 & & & & & .743 \\
\hline 13 & & & & & .680 \\
\hline 11 & .308 & & & & .653 \\
\hline 23 & & & & & .620 \\
\hline 17 & & & & .355 & .549 \\
\hline
\end{tabular}

${ }^{\bar{a}}$ Staff development and support. ${ }^{b}$ Program sustainability. ${ }^{c}$ Visionary leadership development.

\section{Multivariate Analysis}

Multivariate analysis of variance (MANOVA) was conducted to determine whether there were any significant statistical differences $(0.05)$ between 4 of the demographic information and the 26 leadership recommended practices. A MANOVA is an extension of the univariate analysis of variance (ANOVA). In an ANOVA, we examine for statistical differences on one continuous DV by an IV; MANOVA extends this analysis by taking into account the multiple continuous DVs and bundles them 
together into a weighted linear combination or composite variable. As such, MANOVA compares whether or not the newly created combination differs by the different groups, or levels, of the IV. In this way, the MANOVA essentially tests whether or not the independent grouping variable simultaneously explains a statistically significant amount of variance in the dependent variable (Rosenblad, 2009).

The demographic questions that were considered as IVs in the MANOVA analysis included: (a) If the leader's academic background prepared him/her for the requirements of their current position with two levels (yes/no); (b) If the leader has received necessary professional development for the requirements of the current position with two levels (yes/no); (c) If the leader is a member of any national/international organization with two levels (yes/no); and (d) geographical region in current position of the leaders (urban, suburban, rural, mountain, frontier, combination of types, other).

The multivariate analysis revealed significant association among membership $(\mathrm{p}<$ .001) (If the leader is a member of any national/international organization); and geographical region in leaders' current position (urban, suburban, rural, mountain, frontier, combination of types, other $)(p<.002)$ in implementation of recommended leadership practices (see Table 16). Comparison of the other considered demographic variables as IVs revealed no other significant differences. Since the IV If the leader is a member of any national/international organization has two levels (yes, no), a one-way between-group ANOVA (0.5) was conducted to identify where the differences occurred. For geographical region in leaders' current position with more than two levels, post hoc analysis was conducted to confirm where the differences occurred between the levels of the variables (Rutherford, 2012). In terms of membership, results for ANOVA revealed 
that leaders who are a member of any national/international organization reported more implementation of the following practices: develop priorities and strategic plans consistent with the vision and mission $(\mathrm{M}=3.1, \mathrm{P}<.02)$; communicate statutes, policies, codes of ethics, and procedures to assist others in understanding the reasons behind decisions and actions" $(\mathrm{m}=2.9, \mathrm{P}<.001)$; and engage in planning and conducting cross-agency training and staff development opportunities $(\mathrm{M}=1.7, \mathrm{P}<.03)$.

Table 16

\begin{tabular}{|c|c|c|c|c|}
\hline Demographic & $\begin{array}{l}\text { Implementation of Recommended } \\
\text { Leadership Practices }\end{array}$ & $F$ value & $\begin{array}{l}\text { P value } \\
(0.05)\end{array}$ & $\begin{array}{c}\text { Mean } \\
\text { difference }\end{array}$ \\
\hline \multirow{3}{*}{$\begin{array}{l}\text { If the leader is a } \\
\text { member of any } \\
\text { national/international } \\
\text { organization }\end{array}$} & $\begin{array}{l}\text { Develop priorities and strategic plans } \\
\text { consistent with the vision and mission }\end{array}$ & 3.347 & $.02 *$ & $\begin{array}{l}\text { Yes }=3.1 \\
\text { No }=2.8\end{array}$ \\
\hline & $\begin{array}{l}\text { Communicate statutes, policies, codes of } \\
\text { ethics, and procedures to assist others in } \\
\text { understanding the reasons behind decisions } \\
\text { and actions }\end{array}$ & 3.380 & $.001^{*}$ & $\begin{array}{l}\text { Yes }=2.9 \\
\text { No }=1.7\end{array}$ \\
\hline & $\begin{array}{l}\text { Engage in planning and conducting cross- } \\
\text { agency training and staff development } \\
\text { opportunities }\end{array}$ & 7.363 & $.03 *$ & $\begin{array}{l}\text { Yes }=1.7 \\
\mathrm{No}=1.1\end{array}$ \\
\hline \multirow{2}{*}{$\begin{array}{l}\text { Geographical region in } \\
\text { leaders' current } \\
\text { position }\end{array}$} & $\begin{array}{l}\text { Use data-informed decision making to work } \\
\text { toward improving services }\end{array}$ & 2.584 & $0.0001 *$ & \\
\hline & $\begin{array}{l}\text { Commit to and provide resources for staff to } \\
\text { engage in learning opportunities }\end{array}$ & 1.232 & $.004 *$ & \\
\hline
\end{tabular}

*Significant at the level of .05.

In terms of the geographical region in leaders' current position, using Tukey Honestly Significant Difference, Post hoc analysis indicated that the differences were among the suburban and rural directors. Directors in suburban regions reported more implementation of the following practices: use data-informed decision making to work toward improving services $(\mathrm{M}=2.1, \mathrm{p}<.0001)$ and commit to and provide resources for staff to engage in learning opportunities $(\mathrm{M}=2.2, \mathrm{P}<.004)$ than the directors in rural regions. 


\section{Phase II: Qualitative Data Analysis}

Four phone interviews were conducted with state leaders from each of the programs from which Phase I participants were recruited (EI, HS/EHS, EC/ECSE, and Child Find) to ensure representation of at least one state leader from each of those programs. The purpose of the interviews was to answer Research Question 5, "How do state level leaders perceive the leadership implementation, challenges and professional development needs of the district-level and county-level early childhood and early childhood special education leaders?" and explore state leaders' perspectives in terms of the research questions proposed to the district-level and county-level EC/ECSE leaders in Phase I of this study.

A detailed description of the coding process and analyses was provided in Chapter III. In summary, interviews were digitally recorded with the consent of participants. The digital recordings from the individual interview conversations were transcribed verbatim by the researcher. The responses were categorized and coded using NVivo11 (QSR International, 2012). Pertinent phrases from initial responses were coded. As the researcher compiled responses, like responses were coded and then placed into categories for examination. Next, the researcher reviewed key analytic quotations; categories that shared central meanings with other categories were collapsed into unified categories, and their overarching themes were identified. These unified categories represented answers to the key analytic questions as well as key findings/themes.

As explained in Chapter III, investigator triangulation of the data and transcript review were conducted to ensure the trustworthiness and credibility of the qualitative data 
(Brantlinger et al., 2005). Key analytic questions that were used to analyze the perceptions of the state leaders included:

1. What knowledge do the state leaders have regarding the district-level/countylevel EC/ECSE leaders?

2. What knowledge do the state leaders have in terms of evaluation of the district-level/county-level EC/ECSE leaders' performance?

3. What knowledge do the state leaders have in terms of challenges that the district-level/county-level EC/ECSE leaders encounter in order to meet their responsibilities?

4. What knowledge do the state level leaders have in terms of the professional development needs of the district-level/county-level EC/ECSE leaders?

5. What is the state leaders' perceptions regarding the role of higher education in leadership development and capacity building in eth field of EC/ECSE?

The complete interview protocol is provided in Appendix C. The following results are organized by the key analytic questions and key themes that emerged as the result of the analyses.

\section{Analytic Question 1: Knowledge of the State Leaders of District-level/ County-level EC/ECSE Leaders}

The first analytic question focused on the EC/ECSE state leaders' knowledge of the district-level/county-level EC/ECSE leaders in terms of who these leaders were, what their academic and professional background and qualifications were to meet their job responsibilities, and the criteria that was being used to recruit district-level/county-level EC/ECSE leaders. Two main themes emerged that included: lack of clarity in defining 
leadership roles, and lack of rigor in leadership competencies and credentials.

Theme I: Lack of clarity in defining leadership roles. According to the state leaders, in the field of EC in general, there was much diversity in roles and role definitions, and there were different kinds of leadership. The district-level/county-level EC/ECSE leadership roles, responsibilities and, consequently, leadership performance depended on a number of arrangements such as their position's geographical characteristics. All of the state leaders agreed that there was not a database that they could use to look up the qualifications of the district-level/county-level EC/ECSE leaders or directors. Their backgrounds might vary from a bachelor's degree to a Ph.D., and most of the time, it was up to the organizations, not the field, to decide what they wanted in their job description.

The state leaders believed that the district-level/county-level EC/ECSE leadership job requirement oftentimes was just a bachelor's degree in EC or a related field and included broad criteria mainly because of the shortage of workforce in the field. Also, as stated by the state leaders, there weren't any kinds of leadership roles in EC/ECSE programs in the state of Colorado that mandated someone to have a leadership endorsement or license. In almost all instances, the district-level/county-level EC/ECSE leaders had moved into their leadership positions from other positions within the field.

There were a number of professionals who had some type of leadership credential. However, since there was not a formalized standard approach to collect data at the district-level/county-level about EC/ECSE leaders, it was hard to say which leaders in what district or county had what kind of endorsement or credential. According to one of the state leaders: 
There are some leadership type competencies and skills embedded in their (the district level/county-level EC/ECSE leaders) roles, but there is not a role type or title mandated across the state for which a specific leadership type of degree or license is required. So, whether someone has the EC/ECSE leadership title is really a function of the experience or credential that they have obtained in their own career path leading them into that role.

The state leaders also mentioned that even if there were leadership credentials listed in the district-level/county-level EC/ECSE leaders' job description, they were usually broadly defined. Oftentimes, in the urban area school districts the leadership and administrative positions were on a higher pay scale, and they were mainly in charge of coordination of the programs, whereas in smaller rural districts there was one coordinator who was in charge of all the activities and who had a wide range of responsibilities.

Theme II: Lack of rigor in leadership competencies and credentials. Three of the leaders criticized the impact of the workforce shortage in the field of EC, especially in rural areas, where program leaders and directors lack the leadership competencies and credentials in order to do their jobs effectively. According to state leaders, even in state level leadership positions, there was not a requirement for leaders to meet competencies or credentials around leadership specifically, and that was similar to the districtlevel/county-level EC/ECSE leadership. According to two of the state leaders, there were more "teacher leaders" than "administrative leaders" with relevant experiences and background. As one of the state leaders stated:

We see teachers who do a really good job in the classrooms and then leaders and organizations may say, “Oh! you're doing such a great job! We have an opening 
as the education manager for you to go!” And they don't have a leadership background until they're put in these positions. Because they're doing a good job as a teacher. Being a good teacher, maybe you're a good teacher leader, but you are not an organization leader or program leader to meet all the leadership competencies that are required for that job.

All state leaders believed that although it sounded a bit tricky to define what is meant by leadership in the field, they need more leaders who take the time to understand the system and the vision and philosophy of the field and the methodologies in which services are provided to children and their families. The state leaders also thought that they need more local leaders who are constantly looking for knowledge, evidence-based practices and improvement in their performance and programs. According to the state leaders, they lacked EC/ECSE leaders who were: (a) competent at putting their knowledge into practice, (b) effective improvisers, (c) able to provide reflective supervision and grow their staff into leaders, and (d) skilled at outreach with their community partners. However, the state leaders noted that because the majority of the district-level/county-level EC/ECSE leaders had moved into their leadership and management positions from different non-leadership and non-related educational backgrounds, even some just being a parent of a child with special needs, having those expectations was far-fetched.

Two of the leaders also thought that now the field of EC was focusing only on a very small subset, which is regular education classroom teachers. The main purpose is to increase the pipeline of teachers into the field of EC in general, rather than focusing on specialized credentials or licenses for leaders or even individuals who work directly with 
children who have an identified disability. As one of the state leaders mentioned: "We are becoming more of if you have a bachelor's degree, we'll take you! And it would be ok if they don't know child development or the science of brain development. I think that can be detrimental to programs."

According to these leaders, although preparing more teachers in the field is an absolutely essential piece to be thinking of and planning for, the field also needs to move onto other parts of the workforce preparation and think and plan about other critical aspects like leadership and administration. One of the state leaders said:

The question that we need to ask is "What are we focusing on?" We're focusing on classroom teachers. Yes, it's super important, but at the same time, we also have to sort of stretch it out a little bit more so that we can focus on administrators and higher education and teacher workforce preparation programs for that matter. Most of the time, teachers don't leave their job because of the teaching, they leave their job because of leaders or the lack of quality leadership in their school or organizations, and I think that's critical.

When they were asked about their knowledge of any initiatives in the state of Colorado to invest more on the leadership component in EC workforce preparation programs, two of the state leaders talked about initiatives with teacher licensure in the state system to include a small leadership component. However, according to them, those initiatives tied into the issue of educating the "teachers as leaders." One of the state leaders also mentioned having some knowledge about symposiums for elementary principals to gain a foundation in early childhood, which she doubted many responded to because of high expenses to attend those symposiums. 


\section{Analytic Question 2: Knowledge of the State Leaders of District-level/ County-level EC/ECSE Leaders' Leadership Performance}

The second analytic question aimed to explore if the state leaders conducted any kind of evaluation regarding the district-level/county-level EC/ECSE leaders' performance. The main theme that emerged represented lack of accountability and unity. In terms of formal evaluations, all state leaders said that they did not conduct or receive any type of evaluation or assessment reports as a part of the formal accountability in the state education system. There were not any regulations or rules for these state leaders to conduct an annual performance evaluation for the district-level/county-level EC/ECSE leaders. According to these leaders, performance evaluations happened at local levels, and local organizations determined what would be best suited for them. For example, for HS programs, since they were a part of school districts, the evaluation could include the principals of schools doing the evaluation in terms of annual assessments or performance reviews. Two of the state leaders believed that if any local evaluations were conducted in EC leadership, it should be mostly guided by the K-12 system that requires evaluations to happen in a very specific way, using certain kinds of competency rubrics and also accounting for things like students' performance on standardized tests. One of the state leaders emphasized the need for a more unified, state-wide evaluation system that is specific to the realities of the field of EC/ECSE. She stated that:

We have 170 school districts! That being said, there's 170 ways to do things and although the Department of Education might say here's the rules and regulations, we have a huge book of rules and regulations, the districts relinquish. The districts prefer to do their own thing. Oftentimes, we're not always talking together. 
Another state leader acknowledged the importance of doing such evaluations to improve leadership capacity building by enabling the leaders to self-evaluate their performance and to develop plans for improvement. This state leader said:

We do a couple of yearly meetings across the state and a lot of the conversations are related to both recognizing the leadership responsibilities that EC coordinators can take on as well as providing them with skills to self-evaluate their practices and work more effectively in terms of teams, and do continuous quality improvement.

Other state leaders also emphasized the value of leadership evaluations by stating that leadership assessments can help leaders and directors be very self-reflective on what their leadership style is. According to the state leaders, leadership evaluations also impact the local leaders' interaction with other partners for which there is no standard practice in the field. To these state leaders, the evaluations also would help them to better recognize the need for providing support for new EC/ECSE directors at local levels, so that they could become confident leaders who did not struggle to learn skills relevant to their responsibilities. Two of the state leaders talked about initiatives that existed in their administrative units to work on these evaluation priorities at state level. One of the leaders also mentioned the fragmentation in the field of EC and the workforce from different backgrounds who did not specifically have relevant licensure which made any kind of evaluation inconsistent. According to this leader:

I think because our system right now is so variable meaning that not every position has to be filled by someone with an early childhood license, let alone early childhood leadership or administrative license, I'm not sure if evaluation 
reports would help us determine the effectiveness of those who are in charge of leadership or administration positions.

\section{Analytic Question 3: Knowledge of the State Leaders of District-level/ County-level EC/ECSE Leaders' Leadership Challenges}

The third analytic question focused on the state leaders' knowledge of the challenges that the district-level/county-level EC/ECSE leaders' encounter in their leadership positions. To reflect on the challenges of local directors, two main themes emerged that included dealing with multiple roles and lack of collaboration.

Theme I: Dealing with multiple roles. Three of the directors thought that the lack of time and having to deal with multiple responsibilities, especially in rural areas was one of the challenges that they heard from the district-level/county-level EC/ECSE leaders. This issue consequently led to the lack of opportunities for the local leaders to invest in the leadership aspect of their position and, instead, do more maintenance activities, considering the fact that according to the state leaders, the district-level/countylevel EC/ECSE leaders had difficulties with managing the business side of what they were doing. One of the state leaders mentioned:

The leaders are actually more in classrooms and on the ground, helping and supporting or they are doing the work that they should be doing, which then takes away from them to do actual leading job and understanding policy and talking with local officials and bringing communities together. They are so busy ensuring just day-to- day operations that they don't have the ability to learn to lead and I think that's a big issue. 
According to the state leaders, for the district-level/county-level EC/ECSE leaders, serving in multiple roles divided their attention and resulted in the lack of quality and consistency in their performance. This issue seems inevitable considering the fact that they were performing multiple roles. The district-level/county-level EC/ECSE leaders might directly serve children or they may have a coordinator role with different responsibilities such as identifying children for eligibility for special education, evaluating children directly, coordinating the intake process, and coordinating with other agencies or other people within their school system to ensure that children get access to services.

Two of the leaders questioned the skillsets of the district-level/county-level EC/ECSE leaders from diverse backgrounds who dealt with these different responsibilities. According to the state leaders, the roles that the local EC/ECSE leaders filled required more specialist knowledge and skillsets, specifically the ECSE responsibilities, and districts were unable to distinguish between the specialists in terms of hiring for specific leadership roles or positions. Two of the state leaders believed that if you read the EC/ECSE requirements, you would see that most of the broad leadership skills are embedded in those job or role descriptions. However, they were not sure if human resource individuals or hiring managers really understood the differences between general EC versus ECSE leadership credentials that are required of leaders who are ready to successfully fulfill the requirements of the position. One of the state leaders stated: "I wonder whether those individuals coming out of the master's program who completed the license requirements actually have an appropriate level of skills." 
Theme II: Lack of collaboration. According to three of the state leaders, the reason for such narrow emphasis on specific requirements of being either an EC or ECSE leader somehow went back to the broad focus on the workforce and early childhood qualifications in the field. To these state leaders, it seemed that often the people who make the decisions for such requirements represent EC in general and don't specifically represent special education or early childhood special education. Most of the decisions and conversations seemed to focus on EC teaching and child care licensing, rather than including special education as well. One of the state leaders mentioned:

We need to represent the ECSE piece in our state level conversations to recognize what some of the needs might be for supporting those ECSE directors already in the field to continue to grow and learn to meet current challenges in terms of serving children and making systems changes that improve our system.

Two of the directors also thought that the lack of knowledge and, consequently, skills and expertise when it comes to young children's education and development, especially in small communities and rural areas, was another challenge that the EC/ECSE local directors faced. According to the state leaders, one of the reasons for the poor competence among EC/ECSE local leaders was rooted in the fact that many of the communities were composed of multiple counties and multiple school districts, which made it challenging for local leaders to interact with one another and to improve their understanding of the successful practices. It was also challenging for state leadership to reach out to local EC/ECSE leaders to make sure that everyone understood what was expected of them under their specific responsibilities. One of the state leader noted: 
We do need more collaborative relationships and some of these (school districts) are really spread out geographically so that's just challenging in and of itself to make sure you have that really collaborative relationship with all the agencies that you need to interact with, and it becomes more challenging when we need to interact with families that have children with special needs or disabilities and what that means for local leaders is that they have to build relationships with all those other programs, you know, human services or child abuse treatment and neglect referrals and Head Start and with any other program that supports young children and families.

Three of the leaders talked about regional meetings that they either did attend or planned to attend in order to be in touch with the local leaders and to become more informed about their needs and challenges. According to these leaders, the regional meetings would strengthen the communication and help build collaborative relationships among state and local leadership and reduce the gaps in the quality of local leadership.

\section{Analytic Question 4: Knowledge of the State Leaders of District-level/ County-level EC/ECSE Leaders' Professional Development Needs}

The fourth analytic question focused on the state leaders' knowledge of the professional development needs that the district-level/county-level EC/ECSE leaders' had to better lead their programs. Two main themes emerged including: individualized PD provision and improving the competence of the workforce.

Theme I: Individualized professional development provision. To reflect on the PD needs of the district-level/county-level EC/ECSE leaders, three of the state leaders built on what they mentioned earlier in their conversations regarding the regional 
meetings they did attend or planned to attend to better target the district-level/countylevel EC/ECSE leaders' challenges and concomitantly their PD needs. In order to become more effective with a variety of local leaders' challenges, the state leaders mentioned the effort they put into aligning the PD with those challenges as much as possible. According to three of the state leaders, the primary way to learn about the PD needs of the districtlevel/county-level EC/ECSE leaders was getting feedback in their regional meetings, which really informed not only the future regional meetings, but also their PD offerings. However, they emphasized the fact that in order to be more effective with the local leaders' needs, they have to become more individualized and that was what they were trying to work more systematically on. As one of the state leaders mentioned:

We have lots of professional development modules and trainings that is available that we've developed and it's all online so that you don't have to travel to access them. So, I would say those are the things in which we provide tools to develop leadership at the local level. They also link people with natural resources such as the connect modules, white papers that come out, and we pass all those resources along, but I think that what is going to be one of our most effective initiatives is regional individualized supports, and we are working on that.

Two of the state leaders also talked about the feedback they received the previous year at the regional meetings about the topics the local leaders identified for improving themselves. They also talked about the effort they put in providing opportunities for teams of local leaders to participate in yearlong PD projects and to engage the local leaders in developing and guiding action plans throughout the year as well as ongoing coaching that was related to those projects. The state leaders stated that they often hear 
about myriads of topics that need to be individualized as the reality of the EC/ECSE leadership and coordination includes one local leader in charge of multiple responsibilities. One of the state leaders noted:

It's more of a challenge for the state leadership since we are facing the local leaders who may be the only person or one of the few people in their entire system that really coordinate the programs, and it might not align with their neighbor who is coordinating in the other district or county near to them with different needs and that makes me to be more individualized when planning to provide such PD opportunities.

Theme II: Improving competence of the workforce. Here again, two of the state leaders expressed their concerns regarding the quality of the workforce preparation programs and the knowledge and skill of the graduates who came out of the programs. According to these state leaders, many of the local leaders enter the administrative positions with different backgrounds that did not require them to have appropriate depth of knowledge and skills in areas related to child development and education. However, by remaining in the field and gaining experience, these leaders ultimately improved their knowledge and skills to meet the requirements of their positions. However, considering the high turnover in the field, it seemed like a weak possibility. One of the state leaders mentioned:

I'll just use myself as an example. I came out of one of the master's level programs and completed all the coursework for endorsement. I did quite well because instruction or intervention, and evidence-based interventions was enough 
of a focus in that program. I am not sure about the same rigor in other teacher preparation programs.

Another state leader also had the same argument and emphasized the lack of leadership-development-specific content in EC/ECSE workforce preparation. According to this leader, leadership development was an absent component in the majority of the work force preparation programs, and most of the leaders learned about being a leader or an administrator while serving in their position. As this state leader said:

It disappoints me to say that graduates are not competent when it comes to their skills and knowledge to meet the needs of children in general, either as a teacher, a practitioner, a professional. Now if we add leadership as a weak component to these teachers' or workforce preparation programs, I become more hesitant to think about the local leaders' skillsets in meeting their job requirements.

To these state leaders, making sure of the competence and skills of the districtlevel/county-level EC/ECSE leaders in a way that they are well-equipped to meet the requirements of their position was a priority in providing local and statewide PD opportunities. However, lack of communication and, thereby, knowing about their needs and lack of resources in providing for such needs were among the issues that the state leaders also mentioned while addressing the PD needs of the district-level/county-level EC/ECSE leaders. One of the state leaders also mentioned the need for more skills around reflective supervision for the district-level/county-level EC/ECSE leaders so that they could become more independent and more of a "leader," rather than an administrator to benefit their programs. As this state leader noted: 
I would really love to see programs embed reflective supervision into some of their practices. Again, though, that means that I need to have skills to know how do I reach out to my community to facilitate such reflective supervision activities, but I think that would be a very helpful tool for our local leaders to get out of their comfort zone and better lead the local EC/ECSE programs.

One of the state leaders also mentioned diversity as one of the areas that needed more targeted planning and PD provision as it had not been addressed properly within leadership roles and positions in EC/ECSE. According to this leader, bringing diversity into the conversations of state leadership was what she was trying to do so that more attention was paid to promoting diversity among the leaders and also providing more PD that focused on diversity in general. As this leader stated: "The diversity piece matters a lot since we have leaders that represent the children in their programs and the teachers that they're also supervising. This is a challenge in Colorado, and we need to work on it more seriously."

All of the state leaders agreed that PD opportunities should focus on elevating the role of EC/ECSE leaders and making sure that they had enough understanding of leadership competencies, child development, and education and to value parent partnership to better collaborate and coordinate towards working in the system as a whole, rather than leading individual programs. 


\section{Analytic Question 5: State Leaders' \\ Perceptions Regarding the Role of \\ Higher Education in Leadership \\ Development in the Field}

The last analytic question explored the state leaders' perceptions regarding the role of higher education in leadership development in the field of EC/ECSE. The main theme that emerged was leadership capacity building.

Three of the state leaders emphasized the fact that there was a need to have a specific early childhood leadership certification or an addition of a leadership component which is embedded within the scope and sequence of EC/ECSE degree programs to prepare potential leaders for these roles. According to these state leaders, for most of the child care licensing there was a requirement to have a director-qualified person, which highlights the need to ensure that we have a more robust leadership component in director qualifications licensing. Three of the state leaders also believed that higher education agencies should be a part of their communities and align their programs with the needs of those communities, and such understanding and alignment happens when communities and higher education programs start collaborating and partnering within and across the systems. One of the state leaders mentioned:

We need higher education agencies that are really understanding and are involved in everything within EC/ECSE service systems so that they're part of the decision makers on our advisory councils that help us determine how things are going to look for in our units, how we're going to implement federal laws and how we're going to support our local communities.

According to these state leaders, there were many programs for EC/ECSE professionals and practitioners that did not provide any level of understanding of how 
these roles require a strong partnership with schools, families, and communities. To these state leaders, working in the field of EC/ECSE, specifically birth through 3, which is referred to as EI services in the state of Colorado, is a totally different kind of service and requires a different kind of preparation than the K-12 system as well as different levels of commitment and understanding. One of the state leaders stated:

I think that, when we have folks that come out of higher education programs where they have been exposed to proper understanding, they are ready to hit the ground running! We have folks that are coming into the filed, who have only had experience in more medically-based and clinically-based models of service provision, not educationally or developmentally-based, and they often are confused and need a lot of support.

The state leaders also thought that when comparing the field of EC/ECSE with the K-12 system, the field of EC/ECSE had fallen behind when it came down to leadership and administration preparation programs. According to these state leaders, such content and emphasis was missing in the field of EC/ECSE where the need is more urgent, considering the various backgrounds from which professionals come into the field. According to one of the state leaders:

From working with coordinators who come through a variety of different types of training programs, it might be speech language pathologist, you know master's degrees if it matters or early childhood special education and occupational therapy or school psychology, I get to speak about what that felt like with their pre-service programs to prepare them for their coordination role and most of the time what I hear is that they don't feel prepared. 
To these state leaders, the fact that the field of EC/ECSE includes a variety of different types of special service providers required them to show stronger leadership skills that went much beyond the individual child and included more of a leadership emphasis than administrative. The state leaders believed that the field needs EC/ECSE leaders who are ready to take on systems roles and improve the system as a whole. However, since such specific leadership programs were currently missing in the field, they thought that the workforce preparation programs needed to be more rigorous to ensure the graduates in the field are learning about the most current and up-to-date content. They also thought that pre-service professionals needed practical placements that supported them in getting good mentoring and becoming strong professionals who enter and stay in the field. One of the leaders mentioned:

Graduates come out of these programs (higher education programs) and encounter difficulties in implementing good practices in the systems that they start working in and so part of it is maybe making sure that their programs include systems level, implementation science type information or things that will help them in changing not just their own practice, but if they're in a leadership position for children, they need to learn to create and support the vision and mission of their programs as well.

Two of the state leaders also thought that it would be most useful for higher education agencies to create customized or individualized support for local leaders to grow in their roles as leaders and not necessarily in the early childhood content or pedagogy and learn how to apply EC knowledge in a broader leadership landscape. According to one of the state leaders: 
The local leaders need leadership mentoring or leadership coaching to really get practical experience, having specialists in those fields, guide and support and coach them in those areas would probably be even more useful than being mentored necessarily by someone who has stronger early childhood content knowledge. Now it's more around navigating policy type issues or surviving a bureaucracy or developing teams to be effective and our local leaders need such support.

According to the state leaders, professionals might understand the importance of leadership skills theoretically, but they really struggle to implement leadership skills. According to state leaders, on the one hand, the higher education workforce preparation programs did not have a strong focus on leadership development and, on the other hand, local leaders were all working so hard that they could not find the time to improve their leadership skills themselves. They also believed that although the need for leadership in the field is well recognized, the state level system does not put the resources needed into supporting leadership development in local levels of EC/ECSE. A summary of the themes and supporting sub-themes are presented in Table 17. 
Table 17

Summary of Themes and Supporting Sub-themes

$\begin{array}{lll}\text { Analytical Questions } \quad \text { Main Themes } & \text { Supporting Sub-themes }\end{array}$

What knowledge do the state leaders have regarding the district level/county level

EC/ECSE leaders?

What knowledge do the state level leaders have in terms of evaluation of the district level/county level EC/ECSE leaders' performance?

What knowledge do the state level leaders have in terms of challenges that the district level/county level EC/ECSE leaders encounter in order to meet their responsibilities?

What knowledge do the state level leaders have in terms of the professional development needs of the district level/county level EC/ECSE leaders?
Theme I: Lack of clarity in defining leadership roles

Theme II: Lack of rigor in leadership competencies and credentials

Lack of accountability and unity

Theme I: Dealing with multiple roles

Theme II: Lack of collaboration

Theme I: Individualized PD provision

Theme II: Improving the competence of the workforce
Diversity in roles and role definitions

No data base for local leaders' qualifications

Local leaders with various backgrounds

Various job requirements and criteria

No mandatory leadership licensure or endorsement

Impact of EC workforce shortage on local leadership

More teacher leaders than administrative leaders Lack of competent in EC/ECSE leadership More focus on EC in general rather than ECSE

The need for more leadership and administration preparation

No state evaluations

Local evaluations guided by K-12 system The need for a more unified evaluation in EC

The need to enable the EC/ECSE leaders to selfevaluate their performance

Lack of leadership and more of maintenance Fragmented attention to any of the leadership roles in local levels

Lack of expertise of local leaders to serve multiple roles

Lack of emphasis on ECSE leadership

Lack of collaborative relationships among local leaders

Lack of empowering relationships with parents

Conducting regional meetings to hear about the local leaders' needs

Aligning the PD provision with the needs

Become more individualized within PD provision

Engage the local leaders in developing and guiding action plans

Lack of knowledge and expertise of workforce More of experiential learning and experience than rigorous preparation

Poor leadership focus and content in pre-service preparation

Lack of communication and resources to align $\mathrm{PD}$ provision with needs

More focus on PD around diversity in EC 
Table 18 (continued)

\begin{tabular}{|c|c|c|}
\hline Analytical Questions & Main Themes & Supporting Sub-themes \\
\hline $\begin{array}{l}\text { What is the state level } \\
\text { leaders' perceptions } \\
\text { regarding the role of } \\
\text { higher education in } \\
\text { leadership } \\
\text { development and } \\
\text { capacity building in } \\
\text { eth field of } \\
\text { EC/ECSE? }\end{array}$ & Leadership capacity building & $\begin{array}{l}\text { The need for a specific early childhood } \\
\text { leadership certification } \\
\text { Empowering more leadership rather than } \\
\text { administration } \\
\text { Partnership between higher education and local } \\
\text { communities } \\
\text { Develop more understanding about EC/ECSE } \\
\text { programs } \\
\text { More opportunities for coaching and mentoring } \\
\text { in leadership skills }\end{array}$ \\
\hline
\end{tabular}

\section{Summary of the Chapter}

This chapter summarized the findings from Phase I and Phase II of the study. In Phase I, using an online three-section survey, the district-level/county-level EC/ECSE leaders reported some demographic information in terms of their academic and professional background as well as the characteristics of their leadership position. The district-level/county-level EC/ECSE leaders also self-evaluated their implementation of recommended leadership practices, the implementation challenges in each of the recommended leadership practices, and the levels of the PD that they needed to better implement those practices. The district-level/county-level EC/ECSE leaders also had the opportunity to add further comments about their perspectives regarding leadership implementation, evaluation, challenges, and PD needs through open-ended questions. In Phase II of the study, EC/ECSE state leaders expressed their understanding and perceptions regarding district-level/county-level leadership implementation, implementation challenges, and PD needs of the local leaders. The state leaders also shared their perspectives around the role of higher education in leadership development 
and capacity building the field of EC/ECSE. The following chapter will review the findings from both the quantitative as well as the qualitative data and will provide conclusions about the findings. 


\section{CHAPTER V}

\section{DISCUSSION}

The final chapter of this dissertation study begins with the restatement of the research problem along with an overview of the study and a summary of the findings from Chapter IV that were derived from both the quantitative and qualitative data. The chapter then discusses the implications related to the significance of the research findings. Recommendations for further research in terms of leadership development and capacity building in early childhood (EC) and early childhood special education (ECSE) are also suggested.

\section{Restatement of the Problem}

Advancing quality and professionalizing EC/ECSE programs will require a strong and diverse cadre of leaders at all levels, capable of driving continuous improvement and facilitating transformative change and innovation (Abel et al., 2018). Yet, the field of EC/ECSE education and care faces a leadership development gap (Bloom, Hentschel, \& Bella, 2016). The dearth of attention paid to leadership development and sustainability in EC/ECSE undermines the field's capacity to design and deliver the education and care that makes a difference for children and their families most in need (Kivunja, 2015).

Low compensation continues to undermine the field in preparing, supporting, and retaining quality leaders. The low wages of the EC/ECSE workforce often force them to leave the field or to seek second jobs, public assistance, or loans to make ends meet 
(Douglass \& Austin, 2017). Limited resources in EC/ECSE tend to go to direct services or entry-level workforce development, without addressing the systematic issues that keep the field from ensuring that all young children have access to high-quality EC/ECSE education and care (Bloom, 2014). Also lacking an intentional and deliberate system in EC/ECSE for cultivating, supporting, and nurturing quality leadership (Bloom et al., 2016). Consequently, investments in the EC/ECSE workforce are diminished when EC/ECSE programs lack experienced leaders at all levels to support and promote a culture of continuous quality improvement (Aubrey et al., 2013).

Additionally, almost no public or private investment targets leadership development in a systematic way in the birth to $5 \mathrm{EC} / \mathrm{ECSE}$ sector, in striking contrast to investments in leadership development in other sectors (Ang, 2012). Little attention has been paid to building systems for developing and supporting the leadership of EC/ECSE professionals to drive improvements. With today's renewed attention to the EC/ECSE workforce, it is critical to build up the leadership development infrastructure (Aubrey et al., 2013). The striking gaps are mirrored in EC/ECSE research literature, which as yet has just scratched the surface on leadership (Abel et al., 2018). Considering that developing leadership in EC/ECSE is a missing component of most of our current approaches to workforce preparation and quality improvement, more research-based initiatives are required to build leadership capacity in the field (Bloom, 2014).

\section{Overview of the Study}

This explanatory sequential mixed-methods research study aimed to investigate leadership practices in terms of implementation, implementation challenges, and professional development (PD) needs by district-level and county-level EC and ECSE 
leaders in the state of Colorado to identify: (a) who the leaders are in terms of their leadership qualifications and background; (b) the current state of leadership implementation; (c) the challenges and barriers that affect the EC and ECSE leaders' performance; (d) the support these leaders need to practice quality leadership development and sustainability in the field; and (e) the state leaders' perceptions regarding leadership implementation, challenges, and professional development needs of the district-level and county-level EC and ECSE leaders. The following research questions guided this study:

Q1 Who leads district-level and county-level early childhood and early childhood special education programs? What leadership competencies and academic and professional background do they have?

Q2 How do district-level and county-level early childhood and early childhood special education leaders evaluate their leadership implementation?

Q3 What challenges do these leaders encounter in leadership implementation and development in their programs?

Q4 What professional development and support do these leaders need to implement quality leadership in their practice?

Q5 How do state-level leaders perceive the leadership implementation, challenges, and professional development needs of district-level and countylevel early childhood and early childhood special education leaders?

A cross-sectional online survey was used in Phase I with district-level and county-level EC and ECSE leaders to explore the first four research questions followed by individual interviews in Phase II with state level EC/ECSE leaders to answer the fifth research question.

\section{Summary of the Findings and Implications}

Considering the findings from both the quantitative phase with the districtlevel/county-level EC/ECSE leaders and the qualitative phase with state-level EC/ECSE 
leaders in terms of the five research questions, the summary and discussion of the findings are guided under three main sections including: (a) district-level/county-level EC/ECSE leadership profile; (b) leadership implementation and practices--barriers to leadership development in EC/ECSE; and (c) state and local leadership alignment and coordination. Below, highlights of the study findings and discussions of the findings are presented.

\section{District-Level/County-Level EC/ ECSE Leadership Profile}

The highlights from the demographic information gained from the districtlevel/county-level EC/ECSE leaders in this study include: the hyper-feminine nature of the leadership at the local level, the lack of diversity among the leaders, and the absence of a specific EC/ECSE leadership license/endorsement which can be considered as the overarching theme from the other main findings in terms of leadership implementation, challenges, and PD needs. Ninety-five percent of the district-level/county-level EC/ECSE leaders identified themselves as females, and $77.3 \%$ of the leaders reported to be White/non-Hispanic/Non-Latino in terms of their ethnicity. Also, most of the respondents (36.4\%) reported having EC or ECSE general licensure/endorsement. About $65.7 \%$ of the leaders believed that their academic background had prepared them for their leadership role, whereas $91.61 \%$ believed their professional background played the main role in preparing them for their leadership position. Additionally, almost half the leaders (46.65\%) stated that they were not receiving the necessary PD for the requirements of their current leadership role. The majority of these leaders $(83.22 \%)$ also did not provide any PD in their current positions. 
As mentioned above, since the findings regarding the leadership preparation and competencies tie closely into the other findings in terms of leadership implementation, challenges, and PD needs, they will be discussed further along with those findings. In what follows, impact of feminism and diversity on leadership development and sustainability in the field are discussed.

Gendered occupation. Notions of leadership within the EC/ECSE sector are additionally complicated by its strongly gendered nature (Davis et al., 2015). Osgood (2010) has argued that EC education may be seen as lacking in professionalism because it is deemed "hyper-feminine." Cultural stereotypes of women as caring and nurturing means that they have always tended to be concentrated in the social welfare, health care, and education professions. The field of EC/ECSE is a particularly gendered occupation because it conflates such definitions with a maternal role, and this has particular implications for the consideration of EC leadership (Davis et al., 2015). Muijs et al. (2004) pointed out that the extent to which women occupy leadership roles combines with the complexity of the field in establishing leadership in the sector as wholly different to that in schools. According to Muijs et al. (2004), such female domination, however, creates difficulties in itself as it can be used as an explanatory device and seeks to locate leadership practice within gender-specific behavior.

There has been some emphasis on identifying and explaining any variations in approaches between male and female leadership (Gipson, Pfaff, Mendelsohn, Catenacci, \& Burke, 2017). Certainly, working in the EC/ECSE sector has long been seen as a lower status occupation than teaching in schools, and the lesser financial rewards help to contribute to a lack of men entering the field (Clark \& Murray, 2012). Dunlap and 
colleagues suggested that the association of women in any roles within the field of EC/ECSE is strongly connected to the expressive characteristics (e.g., collaborative and participatory leadership styles, co-operation, and connectivity) which parents value so highly (Dunlap, Ester, Langhans, \& Fox, 2006). However, there is also concern that increased accountability could overwhelm this caring dimension and result in more technical and instrumentalist approaches. Such approaches are generally associated with more masculine styles of leadership (Aubrey et al., 2013). Henderson-Kelly and Pamphilon (2000) have suggested that traditional models of leadership embodying masculine values and attributes do not easily transfer to the field of EC/ECSE and, therefore, women professionals are developing new styles of leadership based on collaboration and dialogue within non-hierarchical groups which are more appropriate to the sector. This might suggest that EC/ECSE is primarily a female domain and that gender of the workforce is one of the most crucial aspects to consider in relationships to leadership (Muijs et al., 2004).

It is also argued that gender is not a particularly helpful concept in analyzing EC/ECSE leadership because it runs the risk of mis-constructing values within the fields as gendered (Aubrey et al., 2013). This is reinforced by certain feminist perspectives which maintain that women are intrinsically more socially adept, emotionally literate, and collaborative in their nature (Clark \& Murray, 2012). The dangers of using such dichotomies in the leadership framework in the field of EC/ECSE is that they are limiting rather than opening up the possibilities for interaction and might result in great loss of potentials (Henderson-Kelly \& Pamphilon, 2000). Aubrey et al. (2013) warned against the danger of too closely associating characteristics of leadership with masculine or 
feminine values and qualities and suggested that this leads to both stereotyping women and alienating nurturing men.

Diversity. Diversity among leadership throughout the field of education is essential to ensuring educational equity for all children (Clark \& Murray, 2012). Diverse leadership in EC/ECSE may be particularly significant in reversing the current low levels of educational achievement for children from ethnic minorities because this is where children first enter the system (Bloom, 2014). The need for leaders to balance the world views, developmental norms, and codes of conduct of diverse populations in EC/ECSE is receiving more attention than it has in the past (Douglass \& Austin, 2017). Early childhood/Early childhood special education educators and professionals serving children from birth to 5 years of age are the most racially, ethnically, and linguistically diverse sector of the education workforce from birth to postsecondary education in the U.S. (Clark \& Murray, 2012). Programs that actively embrace learning from diverse families provide the most effective support for children's development. They can integrate classroom environments, materials, activities, and other practices or program services with a child's knowledge and experience (Bloom, 2014).

The current disproportion between the culture and ethnicity of traditional leadership in the field of EC/ECSE and that of the children the field serves has limited the representation of the strengths and needs of children with diverse backgrounds and their families (Douglass \& Austin, 2017). The field needs leaders who more closely reflect the cultural, racial, ethnic, and linguistic diversity of the children and families served by the EC/ECSE systems and who have a deep understanding of all children and families and the influence of social and economic forces on families (Clark \& Murray, 2012). Such 
leaders can provide appropriate supports in their programs to create meaningful partnerships with parents in which everyone is working together to ensure that children gain optimal benefits from their EC/ECSE experience (Clark \& Murray, 2012). While some of that leadership can come from empathetic, sensitive individuals from other cultures, there is a need for adults who actually share the culture of the children who can most authentically affirm children's experiences and understand them in their home context. The diversity in the fields of EC/ECSE is an asset, and cultivating diverse leadership is essential to the future of the field (Aubrey et al., 2013).

\section{Implications in Terms of Leadership Development and Capacity Building in Early Childhood/Early Childhood Special Education}

Inclusive leadership development. When thinking about leadership development and capacity building in the fields of EC/ECSE, more inclusive perspectives should be taken and leadership should be built on affective values, not gender-related assumptions; EC/ECSE leadership should not be viewed as women's style of leadership. The field should move toward a more inclusive paradigm of leadership development to prevent undermining professionalism and marginalizing male presence. It will also require the field to work toward elevating the status of the field so that the field can attract men into the leadership positions. Specific implications in terms of organizational development of leadership preparation programs will be discussed further in this chapter.

Diverse leadership development: The efforts to lay the groundwork for more diverse leadership must start with increased educational opportunities for entry- and midlevel EC/ECSE professionals. Institutions of higher education, professional associations, and advocacy organizations must work together to better coordinate existing efforts to 
support the advancement of minority professionals and to develop new strategies for diversifying the field at the leadership level. Building an ethnically representative corps of individuals at various levels will ensure that minority communities will be wellrepresented in all positions. In addition, if we assume that diversity is central to the development of the profession, it is appropriate that a monitoring system be established to ensure that organizations undertake efforts to include minorities at higher levels of the EC/ECSE education and care and offer both formal and informal support to individuals from groups often excluded from leadership roles as well as to promote new leadership models that include diverse cultural perspectives and experiences.

\section{Leadership Implementation and \\ Practices: Barriers to Leader- ship Development in Early \\ Childhood/Early Childhood Special Education}

Leadership vs. management. As stated above, one of the main findings of this study was the vague understanding and few opportunities for local directors to practice leadership vs. management. Both the results from the online survey regarding leadership implementation, leadership implementation challenges, and PD needs, analysis of the open-ended questions and alignment of the findings with the state-level leaders showed that the majority of the district-level/county-level directors had issues with task and responsibilities that required more of management rather than leadership knowledge, skills, and expertise.

As presented in Chapter IV, this issue is mainly rooted in the fact that these leaders do not get targeted preparation and development for leadership in the field to promote them to their leadership position from different backgrounds. This lack of 
leadership development consequently impacts their leadership knowledge, competencies, skills, and expertise. The leaders are put in their leadership positions unprepared, dealing with the myriad responsibilities on the one side and low compensation and low career status on the other side that leaves them to become and remain as managers rather than leaders. Cuban (1988) provided a clear distinction between these two concepts. He linked leadership with change and saw management as a maintenance activity. He also stressed the importance of both dimensions of organizational activity:

By leadership, I mean influencing others' actions in achieving desirable ends. Leaders are people who shape the goals, motivations, and actions of others. Frequently they initiate change to reach existing and new goals. Leadership takes much ingenuity, energy and skill. Managing is maintaining efficiently and effectively current organizational arrangements. While managing well often exhibits leadership skills, the overall function is toward maintenance rather than change. I prize both managing and leading and attach no special value to either since different settings and times call for varied responses. (pp. 34)

Understanding and distinguishing between Cuban's (1988) definitions of leadership and management is essential. In order to lead EC/ECSE programs effectively, leaders need to recognize that they are actively promoting change and improvement to work with and provide support to other people (Douglass \& Austin, 2017). These EC/ECSE leaders need to be able to both lead and manage as well as recognize when each is appropriate.

However, considering the findings of this study in terms of the absence of any targeted leadership preparation in EC/ECSE in Colorado, it is clear that this, along with 
the nature of the local leaders' job in dealing with multiple responsibilities without much support and the low public status of the field of EC/ECSE, is one of the barriers to such leadership development. There are few leadership requirements for EC/ECSE leaders. Child care regulations in most states require that administrators of centers have some EC/ECSE teaching experience or qualifications, but very few require that directors have any leadership or administration preparation. When this relates to EC/ECSE preschool directors, there is a total absence of either preparation or credential requirements (Clark \& Murray, 2012).

Leadership development from within. Developing leadership from within in the fields of EC/ECSE is an untapped area for transforming the EC/ECSE landscape, strengthening our workforce, and ensuring that all young children and their families thrive (Douglass \& Austin, 2017). Few opportunities or systems exist to develop and support leadership, either independently or from within the EC/ECSE workforce. All too often, the EC/ECSE workforce has been seen as the object of change, rather than the architect and co-creator of change (Rodd, 2014).

Unlike many other professionals, experienced early educators lack opportunities to grow throughout their careers and to develop the skills and supports they need to lead in their profession, communities, and beyond. This can result in loss of talent from the field when it is most needed. Today, most children in the U.S. under the age of 5 are cared for in EC/ECSE education settings (Douglass \& Austin, 2017) and cared for 40-50 hours a week, often over a period of several years. The EC/ECSE sector employs more educators than the K-12 education system in the U.S. Early childhood/early childhood special education educators constitute $30 \%$ of the entire instructional workforce from 
birth to postsecondary education (Rodd, 2014). For the field to fulfil its mission and potential, it needs a bold new approach to leadership development and designing models that operate from a set of assumptions different from those of business (Abel et al., 2018).

Research in K-12 education and social innovation sectors proves that leadership from within where educators, practitioners, and professionals are prepared and supported to walk up their career ladders into leadership roles and positions can dramatically improve children's learning (Abel et al., 2018; Bloom et al., 2016). Such leadership has the potential to result in the same dramatic benefits in the EC/ECSE sector. No one is better suited to lead change and improvement than those most involved in the work processes we seek to improve (Douglass \& Austin, 2017). Much attention to the EC/ECSE workforce development focuses on teacher competencies or credentials at the entry level. While this is essential, there is not a comparable focus on how to support and retain experienced EC/ECSE professionals to become prepared to take on leadership roles (Rodd, 2014).

Furthermore, turnover is recognized as a central challenge, and yet PD systems have not been developed to nurture, elevate, and retain the expertise of an experienced EC/ECSE workforce (Aubrey et al., 2013). Strong leadership within the fields of $\mathrm{EC} / \mathrm{ECSE}$ has the potential to raise the status of the EC/ECSE workforce and drive quality improvement, and EC/ECSE leadership from within can act as a catalyzing force to establish equitable compensation. When the fields of EC/ECSE build talent from within the field, they can tap the passion, insight, and leadership in order to revolutionize educational outcomes (Douglass \& Austin, 2017). 
Insufficient support and compensation. According to research findings, the magnitude of responsibilities that local EC/ECSE have, the inadequate pay, and the low occupation status fuels high rates of turnover of the most experienced and skillful professionals preventing them from staying in the field and being promoted in their careers (Douglass \& Austin, 2017; Rodd, 2014). About 40\% of the district-level and county-level leaders in this study stated that they had less than 10 years of experience. Among those who stay, many work second jobs or work exceedingly long hours that made participating in leadership activities difficult or impossible (Abel et al., 2018). Thirty percent of the local leaders in this study stated that they were in charge of other positions besides their leadership roles. The lack of sufficient resources in the field combined with professionals' low earnings result in limited access to PD and participation in leadership capacity-building activities. Many leadership activities such as conferences include fees that are beyond the financial reach of most EC/ECSE professionals, and they cannot attend unless they can be paid for. Additionally, limited resources in the field make this difficult and all too rare (Clark \& Murray, 2012).

Almost half of the local leaders in this study (49.7\%) stated that they did not attend PD activities by any national entities, and $53.1 \%$ of the local leaders reported not being a member of any national/international organization. Relatively few EC/ECSE professionals maintain organizational ties, which means they have limited opportunities to connect and network within the field. Most organizational options also focus on teachers and practitioners from a wide variety of EC roles in the field which makes it more difficult for the EC/ECSE leaders to make their voices heard among a competing chorus of other interests. Also within local sectors, EC/ECSE leaders often constitute a 
small minority of the workforce membership which makes them struggle to raise their voice (Abel et al., 2018; Douglass \& Austin, 2017; Rodd, 2014).

\section{Implications in Terms of Leadership Development and Capacity Building in Early Childhood/Early Childhood Special Education}

New definitions of and visions for leadership in early childhood/early childhood special education. The field has been unclear about what is meant by leadership and where it can and should exist. The field needs to broaden its understanding of what leadership is and how it is best achieved. Leadership should be understood in a way that includes raising awareness in the EC/ECSE community about the need for and value of a formal leadership system, creating a vision for leadership in the field and obtaining consensus about it, and exploring non-traditional roles for EC/ECSE leaders. Also, developing specific leadership performance standards, accreditation, and credentials in both EC and ECSE would be options for moving ahead in the field. Currently, there are administrative and managerial items in the accreditation criteria in the field of EC in general, but there is not a specific focus on leadership in program improvement in either EC or ECSE systems.

Higher education. The most obvious direct route to develop leaders is through higher education programs. A specific degree program and/or licensure/endorsement in EC/ECSE leadership should be developed in four-year colleges and universities that lead the EC/ECSE workforce to prepare professionals interested in taking leadership roles in the field. Each state or regional group of states should have an articulated set of EC/ECSE leadership programs. Programs could even be offered in community colleges, leading to advanced degree programs at universities. Such programs need to include best 
leadership practices in content knowledge and process based on child and family development to create mechanisms that will systematically nurture and prepare EC/ECSE leaders with a strong knowledge base and leadership skills.

There is also a need to create formal and informal support systems and various approaches to formal and informal in-service preparation in EC/ECSE leadership and to promote research on EC/ECSE leadership and appropriate PD. Leadership development is an ongoing process that should be supported with continuing education through graduate-level leadership education for EC/ECSE professionals. Institutions of higher education should also formalize a leadership structure for the field. Currently, there is no structure or agreed upon process for systematically developing a new generation of leaders for the field. To develop an infrastructure, a plan must be made that delineates leadership standards and credentials and is tied into the service delivery system, coordinated with PD needs, and promoted broadly within the field.

Allied organizations and groups. A number of organizations and groups are concerned about, make policy for, and provide services to young children and their families. If policy and plans for services are to be informed by knowledge and understanding, allied groups must include EC/ECSE leadership in a decision-making capacity. Professional organizations and groups should take the lead to call attention to the field of the importance of leadership when planning programs for young children and families, awaken appreciation for the value of EC/ECSE leadership, and develop strategies for getting EC/ECSE leaders into positions in a number of domains.

Clarifying a professional career ladder. There is a need for collaboration between higher education and professional organizations (e.g., NAYEC and DEC) to 
provide specific formal education, flourish and exert leadership, differentiate the career ladder, and organize a system to encourage and facilitate leadership preparation. Both institutions of higher education and professional organizations should create opportunities for EC/ECSE leaders to extend their knowledge and experiences by providing and seeking funding for EC/ECSE professionals to take part in PD activities in leadership, establishing leadership fellowship programs for individuals with demonstrated leadership capacity, and organizing support groups for leaders facing new challenges.

\section{State and Local Leadership Alignment and Coordination}

One of the research questions in this study aimed to explore the state leaders' perceptions regarding the district-level and county-level EC/ECSE leaders' profiles, evaluations of their leadership practices, challenges in implementing quality leadership and their PD needs. According to the state leaders, although there is not a state-wide data system to inform them about local leaders' leadership competencies and credentials, they all believed that the majority of local leaders are professionals with different backgrounds within the field. This might sound like a strength point based on the idea of leadership from within (Douglass \& Austin, 2017).

However, as state leaders in this study mentioned, considering the absence of any formal preparation or specific certification/licensure in EC/ECSE leadership to prepare these local leaders for their leadership roles as the overarching barrier, there is not much strength in local quality leadership and leadership capacity building in the field. Other barriers that these state leaders mentioned (e.g., lack of accountability and rigor in 
leadership competencies, lack of collaboration within and across the systems, and multiple responsibilities to deal with) are all byproducts of the lack of preparation in leadership development specific to the fields of EC/ECSE. Even barriers related to funding and policy could be handled in a more effective and efficient manner with quality EC/ECSE leaders who have the necessary requisite knowledge, skills, and expertise in EC/ECSE systems and service delivery. Dealing with these barriers at the local leadership level needs support from higher levels of leadership (Abel et al., 2018). A strong state and local leadership alignment and connection is needed for sustainable leadership development and improvement in EC/ECSE in local levels and a cross-agency collaborative coordination of services and capacity building. This can be best achieved by creating a leadership development ecosystem that can facilitate coordination, collaboration and networking among different EC/ECSE programs at different levels. Such ecosystem can act as a catalyst to help local leaders learn and become more visible within upper layers of leadership in the EC/ECSE system and have opportunities to influence decision making that impacts everyday practice settings. The ecosystem also open doors to upper level leaders to become more aware of the local leaders and the practice settings. Below, a more detailed explanation of leadership development ecosystem is presented.

In nature, an ecosystem refers to a community of organisms that interact with one another in a living system. This same concept of nested systems that interact in reciprocal ways has been applied to human contexts as well (Douglass \& Austin, 2017). As described in Chapter I, Bronfenbrenner's Ecological Systems Theory explains how a child's development is influenced by the world around him/her (Bronfenbrenner, 1999). 
We can apply this human ecology framework to the EC/ECSE workforce growth. Just as in other human systems, the EC/ECSE workforce performs their work in the context of multiple influencing contexts, environments, and relationships and develop as professionals and leaders in this way (Rodd, 2005, 2014).

One way to create a strong connection between different levels of leadership on local, state, national, or international levels is creating leadership development ecosystems. A leadership development ecosystem consists of the supports, resources, and policies needed to systematically nurture the development of leadership in the EC/ECSE sector that offers ongoing supports and resources for leadership in the field to thrive from within the EC/ECSE workforce (Douglass \& Austin, 2017). The key components of a leadership ecosystem include: supportive workplace, leadership development programs and policies, access to resources and networks, and funding streams and systems that influence EC/ECSE programs (see Figure 2). 


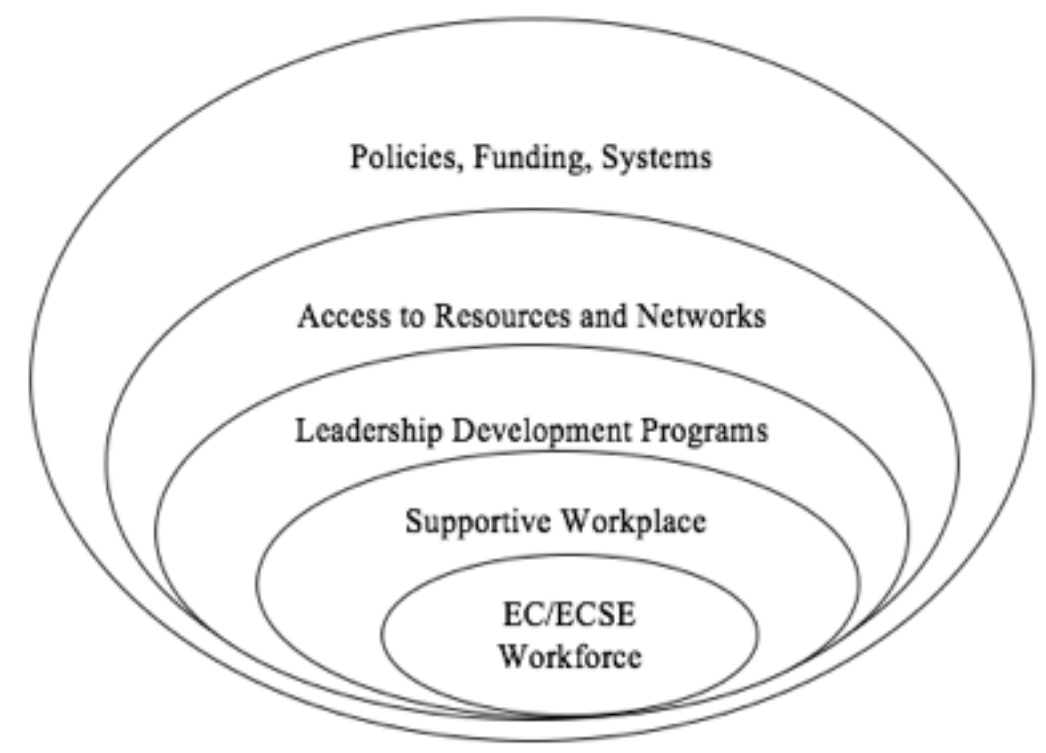

Figure 2. Early childhood/early childhood special education leadership development ecosystem.

Each of these components play a pivotal role in constructing and supporting an ecosystem for EC/ECSE systems. Below, each component is described briefly in hopes that the field considers initiating such an ecosystem.

\section{Implications in Terms of Leadership Development and Capacity Building in Early Childhood/Early Childhood Special Education}

Supportive workplace. The most direct influences on leadership development are found closest to EC/ECSE workforce and professionals. The EC/ECSE workforce needs a supportive workplace that recognizes their potential, creates opportunities to lead, and cultivates a culture of continuous learning.

Higher education institutions play an important role in co-designing these kinds of supports. Including the necessary networks and resources in an ecosystem can enable the 
field to build and strengthen local EC program hubs of innovation and excellence that promote continuous learning (Abel et al., 2016). They need an organizational culture of learning enabled by respectful relationships and supportive structures and policies that foster shared leadership (Abel et al., 2016). As leaders, EC/ECSE directors need more support about how to cultivate these learning environments that make a difference. This points to the importance of an ecosystem that builds organizational capacity for improvement and learning (Douglass \& Austin, 2017). It also fosters an enabling environment for quality improvement through organizational system interventions that enhance coordination, relational leadership, and co-creation (Abel et al., 2016).

The EC/ECSE programs must be connected to an ecosystem that provides the tools and resources they need to create quality conditions for the engaged leadership at local levels. The EC/ECSE systems must also build their capacity to support organizations that effectively engage the leadership from within the field and support the paradigm shift from the current focus on individuals and compliance to one focused on systems thinking and learning (Bloom \& Bella, 2005). As Senge (2006) argued, "Without effective local line leaders, new ideas-no matter how compelling, do not get translated into action and the intentions behind change initiatives from the top can easily be thwarted" (p. 319).

Leadership development programs. Professional development services and programming are the next level of influence in the ecosystem (Douglass \& Austin, 2017). Currently, most PD in EC/ECSE focuses on basic entry-level knowledge and skills, with few more advanced offerings. This is where the field needs to expand leadership development in a way that the EC/ECSE workforce and professionals have access to 
leadership development opportunities provided by experienced professionals (Kagan \& Kauerz, 2012). Higher education can play an important role at this level of the ecosystem by offering undergraduate and graduate coursework on leadership and change including entrepreneurial leadership, policy, and advocacy as well as the science of improvement, creativity, and innovation and systems thinking. Importantly, these programs should be designed for EC/ECSE educators and professionals from within the field who are currently in the workforce (Bloom et al., 2016).

The workforce brings the real-world problems and solutions and connects those in powerful ways with their academic learning. Higher education can build multiple pathways (preservice, early career, and advanced career pathways) to this kind of leadership training in the field (Douglass \& Austin, 2017). State and local professional development systems also can offer similar leadership opportunities. The more these learning opportunities can be closely connected and situated within EC/ECSE programs and settings, the more this will facilitate EC/ECSE workforce capacity to develop quality leadership in the field (Kagan \& Kauerz, 2012). An EC/ECSE leadership preparation and credentialing also must be expanded so more of the workforce has access and is equipped with skills needed for cultivating a culture of improvement and change in their organizations (Rodd, 2005).

Access to resources and networks. Successful, creative and quality leaders need access to the resources that drive change, innovation, intellectual capital, human capital, and financial capital. Intellectual capital is very much the kind of PD and preparation described earlier (Douglass \& Austin, 2017). Human capital refers to the people and networks that a leader needs in order to develop, implement, and test an innovation. 
Financial capital refers to various kinds of funding that can support the development of ideas and testing of innovations (Senge, 2006). Financial capital is a central component of the leadership development ecosystem and must be accessible at the local level and supported at the policy level. These supports enable quality leadership to thrive. This ecosystem would support EC/ECSE leaders to become innovative and turn good ideas into high-impact improvements (Douglass \& Austin, 2017).

Policies, funding, and systems. The EC/ECSE workforce earns low salaries and, therefore, access to scholarships or free leadership development programs is essential. In addition, EC/ECSE leaders need access to financing for facilitating improvements (Abel et al., 2016). Funding should come from both public and private sources. Also, PD and quality improvement policies must be updated to recognize and elevate the needs of the EC/ECSE workforce and stakeholders and understand that the field cannot transform quality if it tries to change only one part of the whole (Kagan \& Kauerz, 2012).

The EC/ECSE profession and policy makers frequently talk about the need to build a more unified system to overcome the disjointed fragmentation of EC/ECSE funding, government agencies, and policies (Rodd, 2014). The field does need a better system. The current system is complicated for providers, expensive for parents, and insufficiently funded and resourced to deliver high quality equitably. However, there are strengths and unique features in the EC/ECSE sector that preserve the capacity for innovation in the field. The EC/ECSE system is not as standardized as the K-12 system, has more freedom to be responsive, and is more nimble and agile in reflecting and responding to the needs of families and children (Kagan \& Kauerz, 2012). This provides quite a bit of room for creativity, innovation, and experimentation in this sector, so the 
field needs to build its capacity to capitalize on those strengths (Douglass \& Austin, 2017).

\section{A Common Vision for Colorado's \\ Early Childhood Leadership \\ Development}

In 2008, EC partners in Colorado identified the need to synthesize and integrate multiple years' system-building efforts into a plan that would provide a collective vision, guide next steps in systems work, and link system-building efforts to outcomes. The Early Childhood Colorado Framework (ECLC, n.d.) was developed to provide that common vision within which both public and private, and state and local level partners could see their work and invest in the future. It connected many pieces of work while outlining the efforts needed by all the partners to make positive changes in the lives of young children and their families (ECLC, n.d.). This framework can be a great starting point to develop leadership in EC/ECSE since it fully recognizes the efforts that must be taken to better coordinate, align, and integrate resources to make EC/ECSE services more efficient and effective for children and families. The mission of the Colorado Early Childhood Framework is for both state and local EC/ECSE systems to promote the development of a comprehensive, sustainable EC/ECSE system that:

- Recognizes the needs of the whole child and family by including outcomes that cut across the early learning, family support, mental health, and health sectors;

- Communicates the vision for comprehensive EC/ECSE work by helping EC/ECSE partners see how their work, individually and collectively, 
contributes to the greater picture of all children in Colorado being valued, healthy, and thriving;

- Connects "comprehensive system-building" language to specific strategies for action and measurable outcomes for children, families, and EC professionals;

- Provides a framework to guide and focus the actions of public and private stakeholders who work with or on behalf of young children and their families;

- Promotes an outcome-based approach to EC/ECSE system-building efforts.

This framework has the potential to guide systematic leadership development and capacity building in Colorado EC/ECSE programs that is child-focused and familycentered, builds on strengths of communities and families, promotes partnerships, and acts at the state and local levels. The framework provides the opportunity to integrate state and local efforts, through: (a) encompassing diverse perspectives and partners across system sectors; (b) providing common language for state and local partners, state and community officials, agency personnel, non-profit leaders, service providers, and family leaders as a common reference to guide planning, discussion, and/or decision-making related to children and their families; and (c) promoting mutual accountability for shared results (ECLC, n.d.). This framework can act as a catalyst for the EC/ECSE systems efforts across a diverse state and numerous partners. Colorado's EC/ECSE leadership should benefit from the unifying vision that this framework provides and promotes shared leadership and ownership to ensure that Colorado's children are valued, healthy, and thriving (ECLC, n.d.). 


\section{Limitations}

As with any research study, there are limitations to this study's findings. First, the study was designed to be cross-sectional, because of the time constraints and the fact that collecting data at a one-time point increases the likelihood of participation. This means, however, that changes in EC/ECSE leaders' beliefs about their leadership practices over time and across programs could not be examined. Second, the current study has limited generalizability because data were collected in only one state. EC/ECSE programs vary in terms of program focus and requirements. In addition, programs vary based on geographic location or the communities in which they are located. In this study, many of the local leaders served in two or multiple agencies. Having participants from multiple EC/ECSE programs provided a broader understanding of how EC/ECSE leadership serves children and families, but limited generalizability in terms of different EC/ECSE programs.

Third, collecting information through self-report measures has some limitations. People are often biased when they report on their own experiences. Self-reports are subject to biases and limitations in terms of honesty, introspective ability (i.e., the participants may not be able to assess themselves accurately), interpretation of the questions, and response bias (questions are subject to all of the biases of what the previous responses were, whether they relate to a recent or significant experience and other factors). Fourth, there are some potential limitations related to measurement. Leadership practices were measured with three self-reporting instruments that used a 5point Likert scale. Participants couldn't explain their responses in-depth, limiting the researcher's understanding of the participants' responses and beliefs in terms of each 
practice. The survey was also adapted from the DEC recommended practices (DEC, 2014) implementation checklist (ECTA, 2016). The measure and its psychometrics should be further evaluated with additional samples of leaders.

Finally, although the sample size was adequate to use as one group, the sample sizes for subgroups (EI, EC/ECSE, HS/EHS, Child Find) limited the types of statistical analyses that could be conducted. For example, the sample size was too small to compare any differences between agencies (e.g., EI directors and EC/ECSE directors). Despite these limitations, the current study provides support for future research and practice to further investigate leadership practices in EC/ECSE programs.

\section{Future Research}

The findings of this research could potentially have implications for the field regarding local leadership implementation, implementation challenges, and PD needs in EC/ECSE programs. However, EC/ECSE programs vary in program focus, state regulations, and system coordination for diverse populations of young children and their families (children with or without special needs or families experiencing low income). Therefore, future research should replicate this study in the context of additional EC/ECSE programs in other states that vary in size, geographic region, and children and family demographics to further the understanding of EC/ECSE leadership practices in the field. The replication of the study using the EC/ECSE leaders from other states and locations would allow for a comparison between the data gathered for this study and other data from a similar study using a different participant group. It would also shed light on the system differences that impact leadership practices in EC/ECSE. 
It seems that the qualitative voice of EC/ECSE state leaders added to the understanding of the survey data, particularly focusing on their perspectives concerning leadership development in EC/ECSE. Future research should examine the local and state leaders' perceptions and practices through more in-depth interviews and on-site observations. Also, more research including the EC/ECSE workforce's perceptions, needs, and perspectives (e.g., teachers, practitioners, and professionals) in terms of quality leadership development and capacity building would add valuable insight to the potential directions that leadership development should take in the field.

Future research also needs to examine existing EC/ECSE programs that do have leadership preparation to better understand how they prepare the EC/ECSE workforce to take on leadership responsibilities and what direction needs to be taken to make those kinds of preparation programs more effective. Another recommendation for further research would suggest using specific program sites with specific quality measures to investigate the leadership practices of the leaders and directors and the relationship of these practices to the program quality. Further research is also needed to understand the leadership behaviors, traits and characteristics that affect EC/ECSE program quality. Another recommendation for future research would be, depending on the main purpose of investigation, to develop and norm valid and reliable measures to explore leadership and produce more reliable evidence. This study can inform the future modification of leadership implementation checklist developed by the Early Childhood Technical Assistance Center that were used to examine leadership implementation, implementation challenges and levels of PD needs. The implementation checklists were basically derived from the DEC Recommended Practices in Leadership, which are currently under 
revision. The findings of this study, specifically the exploratory factor analyses that unravel the underlying patterns of the implementation checklists can inform future research to develop new sets of leadership recommended practices by DEC or any other allied organization in the field.

Finally, more policy development research is needed to help the field elevate its status and push for more legislation that supports benefits for the professionals serving the field to attract, nurture, and retain quality workforce that are prepared and motivate to serve the children and their families.

\section{Conclusions}

The field of EC/ECSE is constantly changing as it continually seeks to improve the ways to serve children and families and support and strengthen the workforce and EC/ECSE systems. Growing public attention to the importance of high quality EC/ECSE programs has added to the pace of change (Kagan \& Kauerz, 2012). In these times of rapid change, it is critical to know how to lead effectively and to improve quality in ways that strengthen the field. There is no need to reinvent the wheel to fix the persistence challenges of quality leadership in the EC/ECSE sector. By applying lessons on leadership and change from across EC/ECSE and other disciplines, the field can learn how to support and nurture a strong, diverse, and resilient leadership and workforce to lead change, improvement, and innovations in EC/ECSE systems.

To do so, the field needs to intentionally invest in cultivating and supporting leadership preparation from within and benefit from the EC/ECSE professionals' unique knowledge and insights that are essential for understanding how to change and improve EC and ECSE systems. It takes more than leadership preparation programs to support and 
sustain quality leadership. The fields of EC/ECSE need to build a leadership and innovation ecosystem to nurture and sustain EC/ECSE leaders throughout their careers and to more fully catalyze action for innovation and change in an ongoing way. The EC/ECSE workforce are uniquely positioned to drive change more rapidly, more effectively, and more sustainably. Local solutions, built from the capacity within the communities, can have a lasting impact. As the field gets to test and adapt and refine new models of leadership development and capacity building in EC/ECSE, we learn more about how to grow it in other communities in ways that retain local ownership while at the same time building connectivity with the larger ecosystem. That way, we can lead transformative leadership that strengthens our workforce and dramatically increases opportunities for all young children and their families to thrive. 


\section{REFERENCES}

Abel, M. B., Talan, T., \& Masterson, M. (2016). Whole leadership: A framework for early childhood programs. The McCormick Center for Early Childhood Leadership at National Louis University. Retrieved from https://mccormickcenter.nl.edu/library/whole-leadership-a-framework-for-earlychildhood-programs/

Abel, M. B., Talan, T. N., \& Magid, M. (2018). Closing the leadership gap: 2018 status report on early childhood program leadership in the United States. Retrieved from https://mccormickcenter.nl.edu/library/closing-the-leadership-gap-2018status-report-on-early-childhood-program-leadership-in-the-united-states/

Allred, K. W., \& Hancock, C. L. (2015). Reconciling leadership and partnership: Strategies to empower professionals and families. Young Children, 70(2), 46-53.

Ang, L. (2012). Leading and managing in the early years: A study of the impact of a NCSL programme on children's centre leaders' perceptions of leadership and practice. Educational Management Administration \& Leadership, 40(3), 289-304.

Aubrey, C. (2007). Leading and managing in the early years. London: Sage Publications. Aubrey, C., Godfrey, R., \& Harris, A. (2013). How do they manage? An investigation of early childhood leadership. Educational Management Administration \& Leadership, 4l(1), 5-29. 
Bailey, D. B. (2000). The Federal role in early intervention: Prospects for the future. Topics in Early Childhood Special Education, 20(2), 71-78. doi.org/10.1177/027112140002000202

Barton, E., \& Smith, B. J. (2015). The preschool inclusion toolbox: How to build and lead a high-quality program. Baltimore, MD: Brookes.

Bays, D. A., \& Crockett, J. B. (2007). Investigating instructional leadership for special education. Exceptionality, 15(3), 143-161.

Bella, J. M. (2013). Discovering and using voice: Characteristics of exemplary directors of early care and education programs who advocate for workforce issues (Unpublished doctoral dissertation). National Louis University, Chicago.

Birt, L., Scott, S., Cavers, D., Campbell, C., \& Walter, F. (2016). Member checking: A tool to enhance trustworthiness or merely a nod to validation? Qualitative Health Research, 26(13), 1802-1811. https://doi.org/10.1177/1049732316654870

Blank, H. (1997). Advocacy leadership. In S. Kagan \& B. Bowman (Eds.), Leadership in early care and education (pp. 39-48). Washington, DC: National Association for the Education of Young Children.

Bloom, P. J. (1992). The child care center director: A critical component of program quality. Educational Horizons, 70 (3), 138-145.

Bloom, P. J. (1997). Navigating the rapids: Directors reflect on their careers and professional development. Young Children, 52(7), 32-38.

Bloom, P. J. (1998). Navigating the rapids: Directors reflect on their careers and their professional development. https://eric.ed.gov/?id=ED420426 
Bloom, P. J. (2014). Leadership in action: How effective directors get things done (2nd ed.). Lake Forest, IL: New Horizons.

Bloom, P. J., \& Abel, M. B. (2015). Expanding the lens: Leadership as an organizational asset. Young Children, 70(2), 8-13.

Bloom, P. J., \& Bella, J. (2005). Investment in leadership training: The payoff for early childhood education. Young Children, 60(1), 32-40.

Bloom, P. J., Hentschel, A., \& Bella, J. (2016). A great place to work: Creating a healthy organizational climate (2nd ed.). Fare Hills, NJ: New Horizons Educational Consultants and Learning Resources.

Bloom, P. J., \& Sheerer, M. (1992). The effect of leadership training on child care program quality. Early Childhood Research Quarterly, 7(4), 579-594. doi.org/10.1016/0885-2006(92)90112-C

Bolman, L. G., \& Deal, T. E. (2013). Reframing organizations: Artistry, choice, and leadership (5th ed.). San Francisco: Jossey-Bass.

Brantlinger, E., Jimenez, R., Klingner, J., Pugach, M., \& Richardson, V. (2005). Qualitative studies in special education. Exceptional Children, 71(2), 195. Bronfenbrenner, U. (1994). Ecological models of human development (2nd ed., Vol. 3). Oxford: Elsevier.

Bronfenbrenner, U. (1995). The bioecological model from a life course perspective: Reflections of a participant observer. American Psychological Association. https://doi.org/10.1037/10176-017 
Bronfenbrenner, Urie. (1999). Environments in developmental perspective: Theoretical and operational models. American Psychological Association. https://doi.org/10.1037/10317-001

Bruder, M. B. (2010). Early childhood intervention: A promise to children and families for their future. Exceptional Children, 76(3), 339-355. doi.org/10.1177/001440291007600306

Bruns, D. A., LaRocco, D. J., Sharp, O. L., \& Sopko, K. M. (2017). Leadership competencies in U.S. early intervention/early childhood special education service systems: A national survey. Infants and Young Children, 30(4), 304-319. doi.org/10.1097/IYC.0000000000000104

Burns, J. M. (1978). Transforming leadership. New York: Harper and Row.

Clark, R. M., \& Murray, J. (2012). Reconceptualizing leadership in the early years (1st ed.). London, UK: Open University Press.

Clifford, R. M. (1997). Personal dimensions of leadership commentary. In S. Kagan \& B. Bowman (Eds.), Leadership in early care and education (pp. 103-104). Washington, DC: National Association for the Education of Young Children.

Coleman, A., Sharp, C., \& Handscomb, G. (2016). Leading highly performing children's centers: Supporting the development of the accidental leaders. Educational Management Administration \& Leadership, 44(5), 775-793. doi.org/10.1177/1741143215574506

Colorado Department of Education. (2019). Colorado preschool program. Retrieved from https://www.cde.state.co.us/cpp 
Colorado Office of Early Childhood. (2019). Early intervention Colorado. Retrieved from http://coloradoofficeofearlychildhood.force.com/eicolorado/EI_Home?lang=en Creswell, J. W. (2007). Qualitative inquiry and research design (2nd ed.). Thousand Oaks, CA: Sage.

Creswell, J. W. (2014). Research design: Qualitative, quantitative and mixed methods approaches (4th ed.). Thousand Oaks, CA: SAGE Publications.

Creswell, J. W., \& Tashakkori, A. (2007). Editorial: Differing perspectives on mixed methods research. Journal of Mixed Methods Research, 1(4), 303-308. doi.org/10.1177/1558689807306132

Crompton, D. (1997). Community leadership. In S. Kagan \& B. Bowman (Eds.), Leadership in early care and education (pp. 49-58). Washington, DC: National Association for the Education of Young Children.

Cuban, L. (1988). The managerial imperative and the practice of leadership in schools. Albany, NY: State University of New York Press.

Culkin, M. L. (1997). Administrative leadership. In S. Kagan \& B. Bowman (Eds.), Leadership in early care and education (pp. 23-37). Washington, DC: National Association for the Education of Young Children.

Culkin, M. L. (2000). Managing quality in young children's programs: The leader's role. Retrieved from https://books.google.com/books/about/Managing_Quality_in_Young_Children_s_ Pro.html?id=tzRyQgAACAAJ 
Daresh, J. (2002). What it means to be a principal: Your guide to leadership. Thousand Oaks, CA: Corwin Press.

Davis, K., Krieg, S., \& Smith, K. (2015). Leading otherwise: Using a feministpoststructuralist and postcolonial lens to create alternative spaces for early childhood educational leaders. International Journal of Leadership in Education, 18(2), 131-148. https://doi.org/10.1080/13603124.2014.943296

Day, C., Sammons, P., \& Gu, Q. (2008). Combining qualitative and quantitative methodologies in research on teachers' lives, work, and effectiveness: From integration to synergy. Educational Researcher, 37(6), 330-342. doi.org/10.3102/0013189X08324091

Dennis, S. E., \& O’Connor, E. (2013). Reexamining quality in early childhood education: Exploring the relationship between the organizational climate and the classroom. Journal of Research in Childhood Education, 27(1), 74-92. doi.org/10.1080/02568543.2012.739589

Division for Early Childhood. (2014). DEC recommended practices in early intervention/early childhood special education 2014. Retrieved from http://www.dec-sped.org/recommend- edpractices

Division for Early Childhood. (2015). DEC position statement: Leadership in early intervention and early childhood special education. Retrieved from https://media.wix.com/ugd/e37417_8e4aaf1293a94fca8e8a127a7658dde7.pdf\#pdf js.action $=$ download

Doherty, G. (2011). The mentoring pairs for child care project: Final outcome report. St. Catherine's, Ontario: Early Childhood Community Development Centre. 
Douglass, A. L., \& Austin, L. J. E. (2017). Leading for change in early care and education: Cultivating leadership from within. NY: Teachers College Press.

Dunlap, G., Ester, T., Langhans, S., \& Fox, L. (2006). Functional communication training with toddlers in home environments. Journal of Early Intervention; Thousand Oaks, 28(2), 81-96.

Dunlop, A. (2008). A literature review on leadership in the early years. Retrieved from LTS Scotland. http://www.ltscotland.org.uk/Images/leadershipreview_tcm4499140.doc.

Early Childhood Colorado Framework. (n.d.). Early Childhood Colorado Framework. Retrieved January 5, 2020, from http://earlychildhoodframework.org/

Early Childhood Technical Assistance Center. (2019). DEC recommended practices performance checklists. http://ectacenter.org/decrp/type-checklists.asp

Early Childhood Training and Technical Assistance System (2019). State/territory profile: Colorado. Retrieved from https://childcareta.acf.hhs.gov/stateprofiles/profiles/CO

Early Childhood Technical Assistance Center \& Center for IDEA Early Childhood Data Systems. (2019). IDEA child outcomes highlights for FFY2016. Retrieved from http://ectacenter.org/eco/pages/childoutcomeshighlights.asp

Ebbeck, M., \& Waniganayake, M. (2003). Early childhood professionals: Leading today and tomorrow. Sydney: MacLennan Petty.

ECTA Center. (2019). DEC recommended practices performance checklists. Retrieved from http://ectacenter.org/decrp/type-checklists.asp 
Epley, P., Gotto, G. S., Summers, J. A., Brotherson, M. J., Turnbull, A. P., \& Friend, A. (2010). Supporting families of young children with disabilities: Examining the role of administrative structures. Topics in Early Childhood Special Education; Austin, 30(1), 20-31. doi.org/10.1177/0271121410363400

Faul, F., Erdfelder, E., Buchner, A., \& Lang, A.-G. (2009). Statistical power analyses using $\mathrm{G}^{*}$ Power 3.1: Tests for correlation and regression analyses. Behavior Research Methods, 41(4), 1149-1160. https://doi.org/10.3758/BRM.41.4.1149

Fixsen, D., Blase, K., Metz, A., \& Dyke, M. V. (2013). Statewide implementation of evidence-based programs. Exceptional Children, 79(2), 213-230. doi.org/10.1177/001440291307900206

Fleming, J., \& Love, M. (2003). A systemic change model for leadership, inclusion, and mentoring (SLIM). Early Childhood Education Journal, 31(1), 53-57. doi.org/10.1023/A:1025188819506

Fonsen, E. (2013). Dimensions of pedagogical leadership in early childhood education and care. Retrieved from https://researchportal.helsinki.fi/en/publications/dimensions-of-pedagogicalleadership-in-early-childhood-education

Fullan, M. (2001). The new meaning of educational change (3rd ed.). New York: Teachers College Press.

Fullan, M. (2004). Leading in a culture of change: Personal action guide and workbook. San Francisco: Jossey-Bass.

Fullan, M. (2000). Change forces: Probing the depths of educational reform. London: The Falmer Press. 
Gasper, M. (2010). Multi-agency working in the early years: Challenges and opportunities. London: Sage Publications.

Gipson, A. N., Pfaff, D. L., Mendelsohn, D. B., Catenacci, L. T., \& Burke, W. W. (2017). Women and leadership: Selection, development, leadership style, and performance. The Journal of Applied Behavioral Science, 53(1), 32-65. doi.org/10.1177/0021886316687247

Goffin, S. G. (2013). Early childhood education for a new era: Leading for our profession. Early childhood education series. New York: Teachers College Press.

Graneheim, U. H., \& Lundman, B. (2004). Qualitative content analysis in nursing research: Concepts, procedures and measures to achieve trustworthiness. Nurse Education Today, 24(2), 105-112. https://doi.org/10.1016/j.nedt.2003.10.001

Green, R. (2005). Practicing the art of leadership: A problem-based approach to implementing the ISLLC standards (2nd ed.). Columbus, OH: Pearson.

Grint, K. (2010). Leadership: A very short introduction. New York: Oxford University Press.

Harper, M., \& Cole, P. (2012). Member checking: Can benefits be gained similar to group therapy? The Qualitative Report, 17(2), 510-517.

Hatch, J. (2002). Doing qualitative research in educational settings. Albany, NY: State University of New York Press.

Hayden, J. (1997). Directors of early childhood services: Experience, preparedness and selection. Retrieved from https://eric.ed.gov/?id=ED408062

Heikka, J., \& Waniganayake, M. (2011). Pedagogical leadership from a distributed perspective within the context of early childhood education. International Journal 
of Leadership in Education, 14(4), 499-512.

doi.org/10.1080/02568543.2016.1179551

Heikka, J., Waniganayake, M., \& Hujala, E. (2013). Contextualizing distributed leadership within early childhood education: Current understandings, research evidence and future challenges. Educational Management Administration \& Leadership, 41(1), 30-44. doi.org/10.1177/174114321246270

Henderson-Kelly, L., \& Pamphilon, B. (2000). Women's models of leadership in the child care sector. Australasian Journal of Early Childhood, 25(1), 8-12. doi.org/10.1177/183693910002500104

Hujala, E. (2004). Dimensions of leadership in the childcare context. Scandinavian Journal of Educational Research, 48(1), 53-71.

Hujala, E., Eskelinen, M., Keskinen, S., Chen, C., Inoue, C., Matsumoto, M., \& Kawase, M. (2016). Leadership tasks in early childhood education in Finland, Japan, and Singapore. Journal of Research in Childhood Education, 30(3), 406-421. doi.org/10.1080/02568543.2016.1179551

Individuals with Disabilities Education Improvement Act. (2004). PL 108-446, 20 U.S.C. 1400-87 (2004).

Isles-Buck, E., \& Newstead, S. (2003). Essential skills for managers of child-centered settings. London: Routledge.

Jennings, P., \& Dooley, K. (2007). Introduction to complex systems leadership theory: An emerging paradigm. In J. K. Hazy, J. A Goldstein, \& B. B Lichtenstein (Eds.), Complex systems leadership theory (pp. 17-34). Mansfield: ISCE Publishing Company. 
Johnson, R. B., \& Onwuegbuzie, A. J. (2004). Mixed methods research: A research paradigm whose time has come. Educational Researcher, 33(7), 14-26. doi.org/10.3102/0013189X033007014

Kagan, S., \& Bowman, B. (1997). Leadership in early care and education: Issues and challenges. Washington, DC: National Association for the Education of Young Children.

Kagan, S., \& Hallmark, L. (2001). Cultivating leadership in early care and education: Reaping the harvest of a new approach to leadership. Child Care Information Exchange, 140, 7-10.

Kagan, S. L., \& Kauerz, K. (Eds.). (2012). Early childhood systems: Transforming early learning. New York: Teachers College Press.

Kagan, S., \& Neuman, M. (1997). Conceptual leadership. In S. Kagan \& B. Bowman (Eds.), Leadership in early care and education (pp. 3-8). Washington, DC: National Association for the Education of Young Children.

Katz, L. (1997). Pedagogical leadership. In S. Kagan \& B. Bowman (Eds.), Leadership in early care and education (pp. 17-22). Washington, DC: National Association for the Education of Young Children.

Keilty, B., LaRocco, D. J., \& Casell, F. B. (2009). Early interventionists' reports of authentic assessment methods through focus group research. Topics in Early Childhood Special Education, 28(4), 244-256.

doi.org/10.1177/0271121408327477

Kivunja, C. (2015). Leadership in early childhood education contexts: Looks, roles, and functions. Creative Education, 06(16), 1710. doi.org/10.4236/ce.2015.616172 
Lincoln, Y. S., \& Guba, E. G. (1985). Naturalistic inquiry. Beverly Hills, CA: Sage.

McCrea, N. L. (2015). Leading and managing early childhood settings: Inspiring people, places and practices. Port Melbourne: Cambridge University Press.

Meloy, B., Gardner, M., \& Darling-Hammond, L. (2019). Untangling the evidence on preschool effectiveness: Insights for policymakers. Retrieved from https://learningpolicyinstitute.org/sites/default/files/productfiles/Untangling_Evidence_Preschool_Effectiveness_REPORT.pdf

Merriam, S. B. (2009). Qualitative research: A guide to design and implementation (2nd ed.). San Francisco: Jossey-Bass.

Movahedazarhouligh, S. (2019, under review). A systematic review of leadership research trends in early childhood and early childhood special education. International Journal of Leadership in Education.

Movahedazarhouligh, S., \& Banerjee, R. (2018). Leadership in implementation of quality family-centered services in early childhood: An exploration of administrators' perceptions, needs and realities. Early Child Development and Care, 3(2), 1-15. doi.org/10.1080/03004430.2018.1503256

Muijs, D., Aubrey, C., Harris, A., \& Briggs, M. (2004). How do they manage? A review of the research on leadership in early childhood. Journal of Early Childhood Research, 2(2), 157-169. doi.org/10.1177/1476718X04042974

Murray, J., \& Clark, R. M. (2013). Reframing leadership as a participative pedagogy: The working theories of early years' professionals. Early Years, 33(3), 289-301. doi.org/10.1080/09575146.2013.781135 
National Center for Education Statistics. (2019). Children and youth with disabilities.

Retrieved from https://nces.ed.gov/programs/coe/indicator_cgg.asp

National Government Association. (2019). Early learning from birth through third grade.

Retrieved from https://www.nga.org/center/issues/early-learning-from-birththrough-third-grade/

National Implementation Research Network. (2019). Stages of implementation analysis:

Where are we? Retrieved from

https://implementation.fpg.unc.edu/resources/stages-implementation-analysiswhere-are-we?o=nirn

Neugebauer, B., \& Neugebauer, R. (2003). The art of leadership: Managing early childhood organizations. Redmond, WA: Exchange Press.

Onwuegbuzie, A., \& Leech, N. (2006). Linking research questions to mixed methods data analysis procedures 1. The Qualitative Report, 11(3), 474-498.

Osgood, J. (2010). Reconstructing professionalism in ECEC: The case for the "critically reflective emotional professional." Early Years, 30(2), 119-133. doi.org/10.1080/09575146.2010.490905

Pace, R. W. (2002). Organizational dynamism. London: Quorum Books.

Preskil, S., \& Brookfield, S. (2009). Learning as a way of leading: Lessons from the struggle for social justice. San Francisco: Jossey-Bass.

Robbins, S. P., Millet, B., Cacciope, R., \& Waters, M. T. (1998). Organizational Behavior. Sydney: Prentice Hall. 
Rodd, J. (1997). Learning to be leaders: Perceptions of early childhood professionals about leadership roles and responsibilities. Early Years, 18(1), 40-44. doi.org/10.1080/0957514970180108

Rodd, J. (2001). Building leadership expertise of future early childhood professionals. Journal of Early Childhood Teacher Education, 22(1), 9-12. doi.org/10.1080/10901027.2001.10486430

Rodd, J. (2005). Leadership in early childhood (3rd ed.). England: New York: Open University Press.

Rodd, J. (2014). Leading change in early years. England: UK: Open University Press. Rohacek, M., Adams, G. C., \& Kisker, E. E. (2010). Understanding quality in context: Child care centers, communities, markets, and public policy. Retrieved from https://eric.ed.gov/?id=ED511767

Rosenblad, A. (2009). Applied multivariate statistics for the social sciences. International Statistical Review, 77(3), 476.

Rutherford, A. (2012). ANOVA and ANCOVA: A GLM approach. Wiley Online Library. http://ebookcentral.proquest.com/lib/unco/detail.action?docID=1011369

Scherpenzeel, A. C., \& Saris, W. E. (1997). The validity and reliability of survey questions: A meta-analysis of MTMM studies. Sociological Methods \& Research, 25(3), 341-383. doi.org/10.1177/0049124197025003004

Senge, P. M. (2006). The fifth discipline: The art and practice of the learning organization (Revised ed.). New York: Doubleday. 
Sims, M., Forrest, R., Semann, A., \& Slattery, C. (2015). Conceptions of early childhood leadership: Driving new professionalism? International Journal of Leadership in Education, 18(2), 149-166. doi.org/10.1080/13603124.2014.962101

Sopko, K. M., \& LaRocco, D. J. (2018). Leading and growing in a culture of reciprocal trust. Young Exceptional Children, 21(2), 63-75. doi.org/10.1177/1096250615621361

Sullivan, D. R. (2010). Learning to lead: Effective leadership skills for teachers of young children. St. Paul, MN: Red Leaf.

Summers, J., Gotto, G., Zuna, N., Marquis, J., Fleming, K., \& Turnbull, A. (2005). Administrative structures in social service agencies serving children and adults with developmental disabilities (Research Brief \#3). Retrieved from ://www.beachcenter.org/resource_library/beach_ resource_detail_page.aspx?intResourceID=2383\&Type=rese archpreview.

Taba, S., Castle, A., Vermeer, M., Hanchett, K., Flores, D., \& Caulfield, R. (1999). Lighting the path: Developing leadership in early education. Early Childhood Education Journal, 26(3), 173-177. doi.org/10.1023/A:1022933502843

Tabachnick, B. G., \& Fidell, L. S. (2013). Using multivariate statistics (6th ed). Boston: Pearson Education.

Talan, T. (2010). Distributed leadership: Something new or something borrowed? Exchange, 2(3), 8-10. Retrieved from http://newhorizonsbooks.net/wpcontent/uploads/2015/06/Distributed-Leadership.pdf 
Talan, T. N., \& Bloom, P. J. (2011). Program administration scale: Measuring early childhood leadership and management (2nd ed.). New York: Teachers College Press.

Talan, T. N., Bloom, P. J., \& Kelton, R. E. (2014). Building the leadership capacity of early childhood directors: An evaluation of a leadership development model. Early Childhood Research \& Practice, 16(1), 10.

Teddlie, C., \& Yu, F. (2007). Mixed methods sampling: A typology with examples. Journal of Mixed Methods Research, 1(1), 77-100. doi.org/10.1177/2345678906292430

Thornton, K., \& Cherrington, S. (2014). Leadership in professional learning communities. Australasian Journal of Early Childhood, 39(3), 94-102.

Toh, Y., Jamaludin, A., Hung, W. L. D., \& Chua, P. M. H. (2014). Ecological leadership: Going beyond system leadership for diffusing school-based innovations in the crucible of change for 21 st century learning. The Asia-Pacific Education Researcher, 23(4), 835-850. doi.org/10.1007/s40299-014-0211-4

Truskie, S. D. (2002). Leadership in high performance organizational cultures. London: Quorum Books.

U.S. Department of Education. (2018). Fortieth annual report to Congress on the implementation of the Individuals with Disabilities Education Act, 2018. Retrieved from https://www2.ed.gov/about/reports/annual/osep/2018/parts-bc/40th-arc-for-idea.pdf

U.S. Department of Education. (2019a). Early learning definitions. Retrieved from https://www.ed.gov/early-learning/elc-draft-summary/definitions 
U.S. Department of Education. (2019b). Race to the top: Early learning challenge.

Retrieved from https://www2.ed.gov/programs/racetothetopearlylearningchallenge/index.html

U.S. Department of Health and Human Services. (2019). Head Start programs. Retrieved from https://www.acf.hhs.gov/ohs

Vandell, D. L., \& Wolfe, B. (2000). Child care quality: Does it matter and does it need to be improved? Retrieved from www.wcer.wisc.edu/childcare/statements.html

Whalley, M. E., Wilson, D., \& Allen, S. (2008). Leading practice in early years'settings. Exeter: Learning Matters.

Wheatley, M. (2006). Leadership and the new science: Discovering order in a chaotic world. San Francisco: Berrett-Koehler.

Woodrow, C., \& Busch, G. (2008). Repositioning early childhood leadership as action and activism. European Early Childhood Education Research Journal, 16(1), 8393. doi.org/10.1080/13502930801897053

Yukl, G. (2013). Leadership organizations. Englewood Cliffs, NJ: Prentice Hall. 
APPENDIX A

PHASE I ONLINE SURVEY 


\section{Operational Definition of Leadership in This Proposed Study:}

For the purpose of this study, an early childhood/early childhood special education leader is defined as a person who is in charge of different leadership responsibilities including administrative, pedagogical, community, advocacy and conceptual leadership.

- Administrative leadership provide programs with vision, inspiration, structure and direction.

- Pedagogical leadership serve as interpreters of research and theory as well as disseminators of new information to parents and other teachers.

- Community leadership reach out to the community and communicate the importance of EC education for the wellbeing of children.

- Conceptual leadership focuses on improving EC education to a more just and equitable society.

- Advocacy leadership get involved in long-term planning and forward thinking and knowledge about the legislative process.

Please answer the following demographic and professional background questions.

Please answer the following demographic and professional background questions.

\section{Section 1: Demographic, Professional and Academic Background}

1. What program(s) do you administer in your current position? Please check all that apply.

a) Early Intervention Colorado Program (Part C, Birth-3)

b) Colorado Preschool Program (CPP)

c) Preschool early childhood special education (IDEA Part B, 619)

d) Child Find (early identification requirements under IDEA)

e) Head Start

f) Early Head Start

g) Other: (Please specify)

2. Please state your employer/agency. Please check all that apply.

a) Colorado Community Board (CCB- Early Intervention Program)

b) School District

c) Private Preschool

d) Private infant/toddler program

e) Early Head Start (EHS)

f) Head Start (HS)

g) Board of Cooperative Education Services (BOCES)

h) Other: (Please specify) 
3. Please state years of experience you have had as an early childhood leader/administer (including this year).
a) Less than 5 years
b) 5-9 years
c) $10-14$ years
d) 15-19 years
e) 20 years and above

4. Please state your gender.
a) Male
b) Female
c) Other:
d) I'd rather not say

5. Please state your age group.
a) $20-29$
b) $30-39$
c) $40-49$
d) 50 and above
e) I'd rather not say

6. Please state the age range of children that your program serves in your current role. Please check all that apply.
a) Birth to 3 years of age
b) Birth through 5 years of age
c) 3 through 5 years of age
d) Other: (Please specify)

7. Please state the highest level of education that you completed.
a) High School or GED
b) Some college course work
c) Associates Degree
d) Bachelor's degree
e) Master's degree
f) Ph.D. or Ed.D.
g) Other (Please specify) 
8. Please state your ethnicity.

a) American Indian or Alaskan Native

b) Asian

c) Pacific Islander

d) Black or African American

e) White/Non-Hispanic/Non-Latino

f) Hispanic/Latino

g) Bi/Multi-Racial

h) Other (Please specify)

i) I'd rather not say

9. Please state the type of geographical region in your current position:
a) Urban
b) Suburban
c) Rural
d) Mountain
e) Frontier
f) Combination of types
g) Other (Please specify):
h) I don't know

10. Please state which of the following licenses/endorsements you currently have. Please check all that apply.
a) K-21 special education generalist license/endorsement
b) Principal license/endorsement
c) Early childhood license/endorsement
d) Early childhood special education license/endorsement
e) Elementary license/endorsement
f) CDHS Child Care Director qualifications
g) Special Education Director license/endorsement
h) Other: Please specify
i) None

11. Do you currently hold any other positions beside your leadership/administrative responsibilities?

a) Yes: Please state:

b) $\mathrm{No}$ 
12. Do you think your academic background prepared you for the requirements of your current leadership responsibilities?
a) Yes
b) $\mathrm{No}$

13. Do you think your professional background prepared you for the requirements of your current leadership responsibilities?

a) $\mathrm{Yes}$

b) $\mathrm{No}$

14. Do you think you have received necessary professional development that prepares you for the requirements of your current leadership responsibilities?
a) Yes
b) No

15. Are you a member of any national/international professional organization?
a) Yes: Please state the organization(s)
b) No

16. Have you attended any professional development hosted by national entity activity in the past two years?
a) Yes
b) No

17. Have you attended any professional development activity within the state of Colorado in the past two years?
a) Yes
b) No

18. Have you provided any PD to state or national level staff in your current leadership role in the past two years?
a) Yes
b) No 


\section{Section 2: Leadership Practices}

Leadership performance checklist is developed based on Division for Early Childhood (DEC) Recommended Practices in leadership and includes examples of steps leaders can take to help create a well-functioning and forward-thinking organization and to help practitioners feel a sense of belonging as they understand their purpose within the organization. The leadership practices include three main sections: (a) vision and direction in leadership, (b) motivation and guidance in leadership, and (c) collaboration in leadership. The checklist can be used as a self-evaluation by leaders at both state and local levels.

Please rate (a) the extent to which you use each of these recommended leadership practices, (b) how challenging you find to apply and implement each of the practices, and (c) the level of training needs that you have in each practice.

\begin{tabular}{|c|c|c|c|c|c|}
\hline \multicolumn{2}{|r|}{ Leadership Practices } & $\begin{array}{l}\text { Seldom or never } \\
(0-25 \%)\end{array}$ & $\begin{array}{l}\text { Some of the time } \\
(25-50 \%)\end{array}$ & As often as I can & Most of the time \\
\hline & Challenges & Not challenging & $\begin{array}{l}\text { Somewhat } \\
\text { challenging }\end{array}$ & Challenging & Very challenging \\
\hline & Training Needs & $\begin{array}{l}\text { Low professional } \\
\text { development need }\end{array}$ & $\begin{array}{c}\text { Medium } \\
\text { professional } \\
\text { development need }\end{array}$ & $\begin{array}{c}\text { High professional } \\
\text { development } \\
\text { need }\end{array}$ & $\begin{array}{c}\text { Very high } \\
\text { professional } \\
\text { development } \\
\text { need }\end{array}$ \\
\hline \multicolumn{6}{|c|}{ Vision and Direction in Leadership } \\
\hline 1 & $\begin{array}{l}\text { Create/revise and/or convey a } \\
\text { vision and mission for the } \\
\text { program derived from } \\
\text { stakeholders who use or are } \\
\text { invested in the system }\end{array}$ & & & & \\
\hline 2 & $\begin{array}{l}\text { Develop priorities and } \\
\text { strategic plans consistent with } \\
\text { the vision and mission }\end{array}$ & & & & \\
\hline 3 & $\begin{array}{l}\text { Create an organizational } \\
\text { culture that values } \\
\text { transparency and } \\
\text { collaborative decision making }\end{array}$ & & & & \\
\hline 4 & $\begin{array}{l}\text { Continue to learn and stay } \\
\text { abreast of knowledge and } \\
\text { research pertinent to work } \\
\text { and share this information } \\
\text { with other colleagues }\end{array}$ & & & & \\
\hline 5 & $\begin{array}{l}\text { Use data-informed decision } \\
\text { making to work toward } \\
\text { improving services }\end{array}$ & & & & \\
\hline 6 & $\begin{array}{l}\text { Advocate for and secure the } \\
\text { fiscal and human resources } \\
\text { needed to provide quality } \\
\text { services and supports }\end{array}$ & & & & \\
\hline 7 & $\begin{array}{l}\text { Understand and communicate } \\
\text { how your program/agency fits } \\
\text { into the larger service system }\end{array}$ & & & & \\
\hline
\end{tabular}




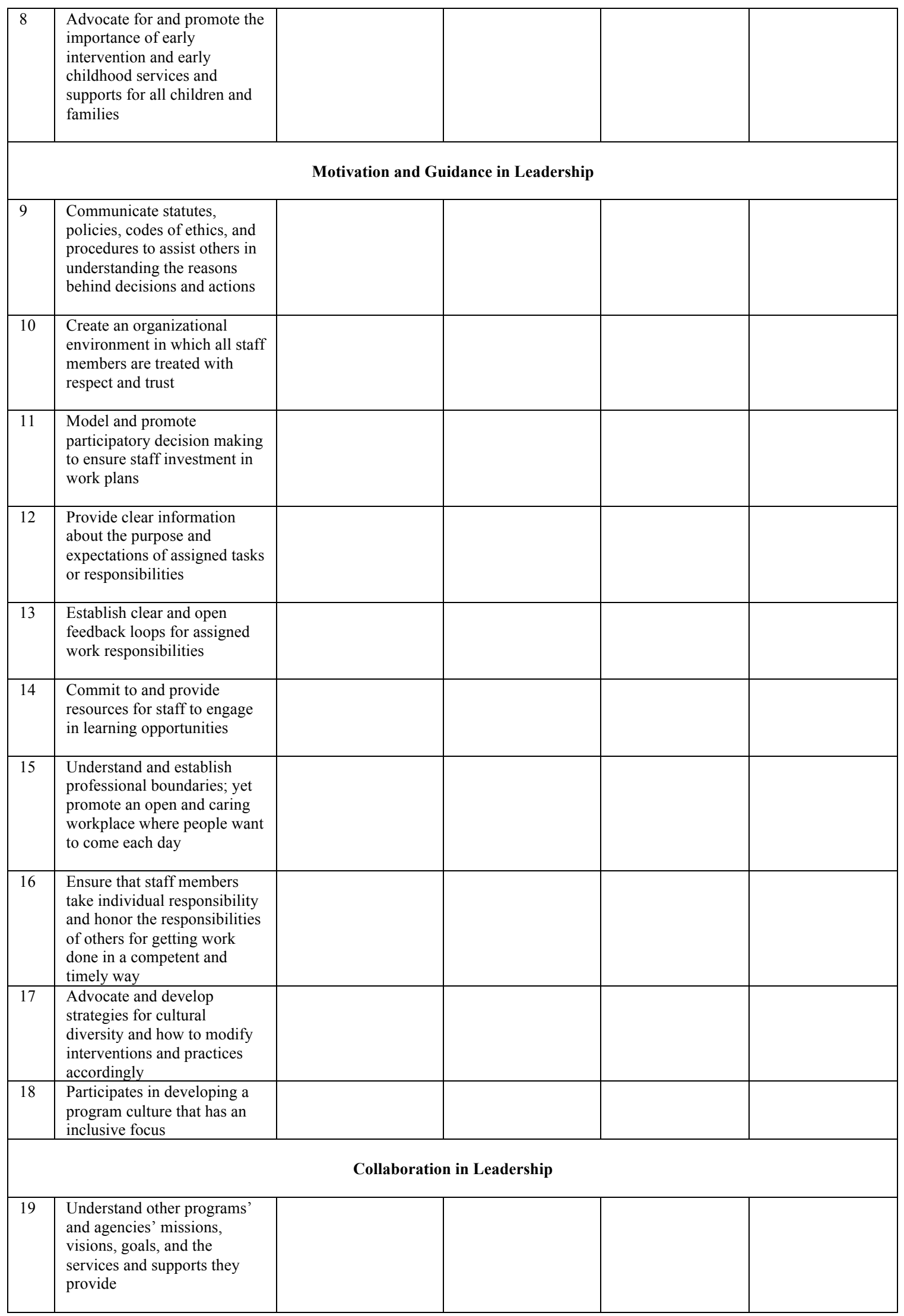




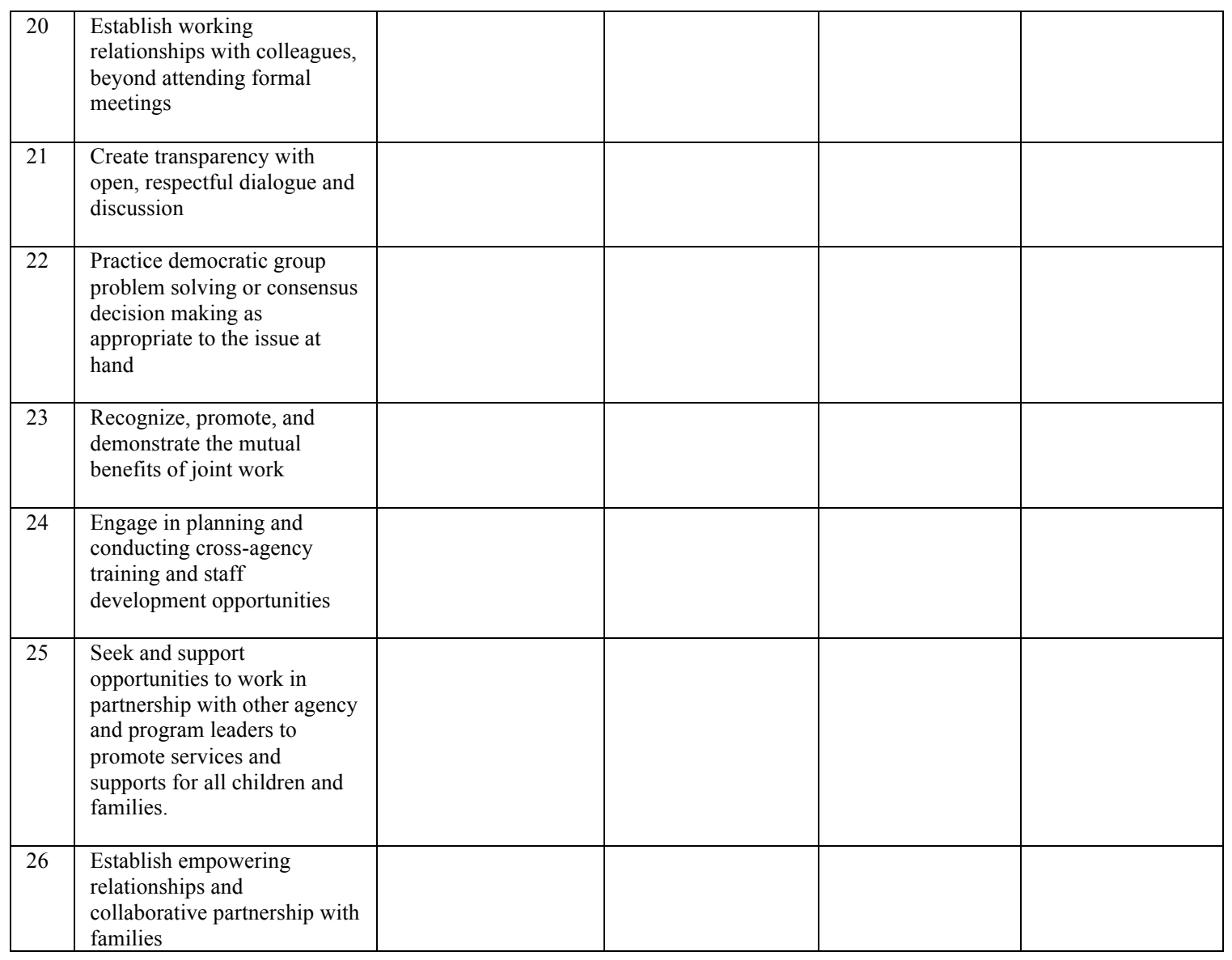

\section{Section 3: Open Ended Questions}

1. Do you conduct any systematic evaluations of leadership practices in your program? Please explain and provide examples.

2. Please state any other comments you have regarding leadership implementation in your practice/program that we have not addressed in the questionnaire.

3. Please state any other challenges you regularly encounter in your role as an EC/ECSE leader that we have not addressed in the questionnaire.

4. Please state any other professional development needs you have in your role as an EC/ECSE leader that we have not addressed in the questionnaire.

5. What else do you think could make a considerable contribution to leadership development and sustainability for you as a leader and in your practice /program? 


\section{APPENDIX B}

CONSENT FORM FOR THE ONLINE SURVEY 


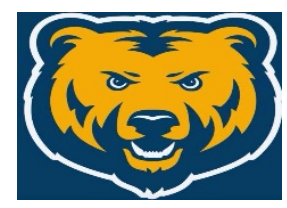

\section{CONSENT FORM FOR HUMAN PARTICIPANTS IN RESEARCH UNIVERSITY OF NORTHERN COLORADO}

Project Title: An Examination of Current Leadership Practices in Early Childhood and Early Childhood Special Education: A Mixed Methods Study

Principal researcher: Sara Movahedazarhouligh, Ph.D. Student, School of Special

Education,

Office: McKee 006

Phone: 970-534-9967

Email: Mova1222@bears.unco.edu

Faculty research advisor: Dr. John Luckner, Ed.D. School of Special Education, University of Northern Colorado

Email: John.Luckner@unco.edu

Phone: 970-351-1672

The objective of the proposed research is to identify (a) current knowledge, skills, and practices of EC and ECSE leaders at district and county levels, (b) the challenges and barriers that affect successful recruitment, development and retention of effective committed leaders in their practice and (c) the support these leaders receive and further need to practice quality leadership development and sustainability. In this study you will be asked to complete an online survey about some demographic and professional background information (e.g., your age, gender, highest degree earned, etc.) and to rate a set of leadership competencies that documents your leadership implementation status, leadership challenges that you encounter and leadership training needs that you have to better implement quality leadership practices in your program. The survey is not expected to take more than 20 minutes of your time. Though there are no direct benefits to you for participating, the data will benefit your profession as a whole and will help institutions of higher education, state departments of education or school districts to plan professional development programs and training programs according to your expressed needs and concerns.

I do not foresee any risk by participating in this study. Your answers and opinions will be kept confidential. The results of the survey will be kept in my personal password protected computer, and no other persons will have access to these data. In order to protect confidentiality of your responses, results will be reported in aggregate form with no personally identifying information. All data will be destroyed three years following data collection. If at any point you wish to no longer participate in the study, you may withdraw without penalty. If you have any questions about the design of the study or your role in the study you may contact me or my research advisor at the email addresses or phone numbers indicated at the top of this consent form. 
Participation is voluntary. You may decide not to participate in this study and if you begin participation you may still decide to stop and withdraw at any time. Your decision will be respected and will not result in loss of benefits to which you are otherwise entitled. Having read the above and having had an opportunity to ask any questions, please be aware that completion of the survey indicates your consent to participate in the study. If you have any concerns about your selection or treatment as a research participant, please contact Nicole Morse, IRB Administrator, Office of Sponsored Programs, 25 Kepner Hall, University of Northern Colorado Greeley, CO 80639; 970-351-1910. 
APPENDIX C

INTERVIEW PROTOCOL 
1 Who leads district level and county level early childhood and early childhood special education programs? What leadership competencies, and academic and professional background do they have?
As a state leader, what knowledge do you have about the district/county level EC/ECSE leaders are?

- Who are these leaders? what is their background?

- What qualifications do they have or required to have? Do their qualifications meet their job responsibilities/requirements?

- What criteria is being used to recruit these leaders?

- What else do you know about their qualifications, academic and professional background?

2 How do district level and county level early childhood and early childhood special education leaders evaluate their leadership implementation?

3 What challenges do these leaders encounter in leadership implementation and development in their programs?
According to your knowledge how are these leaders' performance and leadership practices evaluated either at district level or state level?

- Are any leadership assessment being done on the district /county level leaders' performance?

- If yes, are the findings reported to you? How? How often?

- Do you ("you": the system at the state level) conduct such assessments as a state level leader/official?

To your knowledge, what challenges do the district level/county level leaders encounter in order to meet their responsibilities?

- How do you know about their challenges?

- What steps have you ("you": the system at the state level) taken (or are you planning to take) to reduce these challenges? 
4 What professional development needs and support do these leaders receive, and further need, to implement quality leadership in their practice?

5 General State level leaders' perception

6 General State level leaders' perception
What is your knowledge of the training professional development needs that these leaders have?

- How do you know about their needs?

- What steps have you ("you": the system at the state level) taken (or are you planning to take) to reduce these challenges?

- What steps have you ("you": the system at the state level) taken or are planning to take to meet their needs?

What is the role of higher education and EC/ECSE workforce preparation programs in leadership development and sustainability in the field?

Do you have any additional comments that you think impacts leadership quality, development and sustainability in local levels? 
APPENDIX D

CONSENT FORM FOR PHASE II INDIVIDUAL INTERVIEWS 


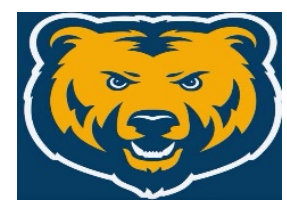

\section{CONSENT FORM FOR HUMAN PARTICIPANTS IN RESEARCH UNIVERSITY OF NORTHERN COLORADO}

Project Title: An Examination of Current Leadership Practices in Early Childhood and Early Childhood Special Education: A Mixed Methods Study

Principal researcher: Sara Movahedazarhouligh, Ph.D. Student, School of Special

Education,

Office: McKee 006

Phone: 970-534-9967

Email: Mova1222@bears.unco.edu

Faculty research advisor: Dr. John Luckner, Ed.D., School of Special Education, University of Northern Colorado

Email: John.Luckner@unco.edu

Phone: 970-351-1672

The objective of the proposed research is to identify (a) current knowledge, skills, and practices of EC and ECSE leaders at district and county levels, (b) the challenges and barriers that affect successful recruitment, development and retention of effective committed leaders in their practice and (c) the support these leaders receive and further need to practice quality leadership development and sustainability. You are invited to take part in an individual interview. You will be asked some questions about your views and perspectives regarding district level and county level EC/ECSE leadership implementation status, leadership challenges that those leaders encounter and leadership training needs that district level and county level leaders have to better implement quality leadership practices in their program. The interview is estimated to take between 45 minutes to one hour of your time. Though there are no direct benefits to you for participating, the data will benefit your profession as a whole and will help institutions of higher education, state departments of education or school districts to plan professional development programs and training programs according to your expressed needs and concerns.

I do not foresee any risk by participating in this study. Your answers and opinions will be kept confidential. Except the consent forms, which will be stored in a locked cabinet or desk in the office of the researcher, all other data collected including recordings and notes, will be stored in a locked cabinet or desk in researcher's university office or home for three years as required by University of Northern Colorado policy. Any computer containing participant data will be password protected to protect subject confidentiality. Also pseudonyms will be used instead of participants' real names. No other persons will have access to the data, and all data will be destroyed three years after final data collection. 
Participation is voluntary. You may decide not to participate in this study and if you begin participation you may still decide to stop and withdraw at any time. Your decision will be respected and will not result in loss of benefits to which you are otherwise entitled. Having read the above and having had an opportunity to ask any questions, please be aware that completion of the survey indicates your consent to participate in the study. If you have any concerns about your selection or treatment as a research participant, please contact Nicole Morse, IRB Administrator, Office of Sponsored Programs, 25 Kepner Hall, University of Northern Colorado Greeley, CO 80639; 970-351-1910.

Participant's name:

Signature:...$\ldots \ldots \ldots \ldots \ldots$

Researcher's name:

Signature: 
APPENDIX E

INSTITUTIONAL REVIEW BOARD APPROVAL LETTER 


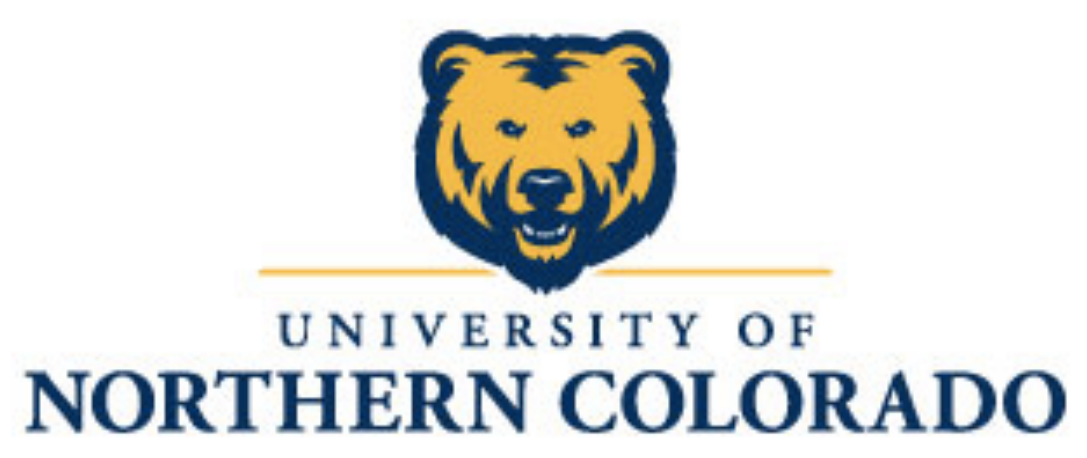

Institutional Review Board

DATE: June 6, 2019

TO: Sara Movahedazarhouligh

FROM: University of Northern Colorado (UNCO) IRB

PROJECT TITLE: [1435086-2] An Examination of Current Leadership Practices in Early Childhood and Early Childhood Special Education: A Mixed Methods Study

SUBMISSION TYPE: Revision

ACTION: APPROVAL/VERIFICATION OF EXEMPT STATUS

DECISION DATE: June 6, 2019

EXPIRATION DATE: June 6, 2023

Thank you for your submission of Revision materials for this project. The University of Northern

Colorado (UNCO) IRB approves this project and verifies its status as EXEMPT according to federal IRB regulations. We will retain a copy of this correspondence within our records for a duration of 4 years. If you have any questions, please contact Nicole Morse at 970-351-1910 or nicole.morse@unco.edu.

Please include your project title and reference number in all correspondence with this committee.

This letter has been electronically signed in accordance with all applicable regulations, and a copy is retained within University of Northern Colorado (UNCO) IRB's records 